\title{
Combining Ability Analysis for Oil and Seed Parameters in Linseed (Linum usitatissimum L.).
}

\author{
Anu Rastogi* and Sudhir Shukla \\ Department of Genetics and Plant Breeding, \\ CSIR-National Botanical Research Institute, Lucknow, U.P., India \\ *Corresponding author
}

\section{A B S T R A C T}

\begin{tabular}{|l|}
\hline K e y w o r d s \\
Linseed, Oil, Acid \\
value, Fatty acids, \\
Colour value
\end{tabular}

The present study deals with the scope of development of linseed lines for edible purposes by estimating its oil, seed and oil quality characteristics. For this study 30 lines and three testers were crossed in line $\mathrm{x}$ tester mating design to get high yielding crosses. Seed colour ranged between greyed orange (165A/166A) or brown group (200D) while majority of crosses in $\mathrm{F}_{2}$ belonged with brown group (200D) except few ones having greyed orange group (165A). Among the testers, Heera was found good general combiner for true density Ex-7959 for porosity and phenol content and Neelam for Iodine value, linolenic acid and linoleic acid. Parents EC-115161 and EC-115178 were good general combiner for oil in $\mathrm{F}_{1}$ generation while CRISTA for in $\mathrm{F}_{2}$ generation. Parent EC-112689 was found good general combiner for oil content in both generations. The crosses EC-104739 x Neelam, EC110288 x Neelam, EC-112689 x Neelam, EC-115178 x Heera and Ex-339-6 x Heera in F1 and crosses EC-41750 x Heera, EC-41752 x Ex-7959, EC-1041492 x Neelam, ES-1474 x Heera and Ezox Heera in $\mathrm{F}_{2}$ generation and cross Ex-28-3 x Ex-7959 in both generations were good specific combiners for oil percentage.

\section{Introduction}

Oils are an essential part of our diet by supplying concentrate source of energy, improving flavor to food and helps in absorption of vitamins in body (Fellows et al., 1998 and Okorie and Nwachukwu, 2014). It can be extracted from a variety of plant seeds such as soybean, cotton, sesame, sunflower, safflower, palm, corn and canola makes them primary oilseed crops. The tremendous research work on these major oilseed crops on various aspects have been done for the development of high oil yielding varieties and are still continuing in India. In spite of sincere efforts, these crops are still unable to meet out the demand and supply ratio of edible oil. So, seeing the growing population rate and their ever increasing demand of oil, there is an urgent need to seek other secondary oil yielding crops having similar edible oil characteristics and nutritional qualities to fulfill this demand. Linseed is one of the oilseed crop, which can be a future edible oil seed crop having physical, chemical, and processing properties similar to other oilseed 
crops with balanced proportion of essential fatty acids except linoleic/ $\alpha$-linolenic acid composition. The commercial demand of linseed is currently dominated by the industrial uses of its oil in varnishes, paints and additives (Dash et al., 2017 and Kaur et al., 2018). The oil is amber in colour and is extracted from the cotyledons and inner coats of seeds. The oil ranges from 33 to $45 \%$ in different varieties having high content of PUFA triglycerides (upto 73\%) including 69\% saturated fat, 16-20\% monosaturated fat, 16-22\% linoleic acid and 60-69\% linolenic acid with vitamins $\mathrm{A}, \mathrm{B}, \mathrm{D}, \mathrm{E}$, minerals, carbohydrates, soluble and insoluble fibers, phytoestrogenic lignans (secoisolariciresinol diglycoside-SDG), proteins, an array of antioxidants and amino acids (Kouba et al., 2003; Basbag et al., 2009; Chauhan et al., 2009;Khan et al., 2010; Herchi et al., 2009 and Goyal et al., 2014).

The whole plant has commercial use directly or indirectly as a food ingredient, industrial purposes and medicinal purposes worldwide (Kumari and Rao, 2008; Bozan and Tamelli, 2008; Bayrak et al., 2010; Gonarkar and Jain, 2013; Biradakar et al., 2016; Singh et al.,2016; Dash et al., 2017 and Kaur et al., 2018). Beside the oil valuable properties, after oil extraction the remaining oil cakes have high protein which are used for baby food, breakfast food, energy rich foods for disaster victims and young stock, manure, fodder, cosmetics, perfumes, insecticides and pharmaceutical products (Biswas et al., 2001; Warrand et al., 2005 and Kaur et al.,2018). The seeds are brown to yellow, flat and oval with pointed tip measuring about $2.5 \times 5.0 \times$ $1.5 \mathrm{~mm}$ and have a pleasant nutty taste, crispy and chewy texture (Gonarkar and Jain, 2013). Generally, oily seeds have greater dimension than fiber seeds (Coskuner and Karababa, 2007). Dried flax seeds are used in cookery as well as for preparation of various medicines for breast and prostate cancers, intestinal and urinary problems, hypercholesterolaemia, thrombosis, platelet adhesiveness and chest problems (Loria, 1993; Guan et al., 1998; Chen et al., 2006; Thompson et al., 2005; Science daily, 2007 and Coskuner and Karababa, 2007). Linseed contains highest arginine and tryptophan when compared with sunflower which is essential amino acids to reducing the healing time of injuries and protein biosynthesis has an amino acid profile comparable to soybean flour and contains no gluten (Hongzhi et al., 2004 and Hussain et al., 2008).

High $\alpha$-linolenic acid content in linseed oil causes sensitivity to oxidation leading to rapid rancidity and reduces its shelf life making it non-edible (Jaswir et al., 2005 andHall et al.,2016). The edible linseed oil with increase keeping and desirable nutritional quality can be obtained by reducing $\alpha$-linolenic acid $<5 \%$ to maintain the required ratio of linoleic and $\alpha$-linolenic acids (5:1 to $10: 1)$ in its oil (Nykter and Kymalainen, 2006 and Ebrahimi et al., 2014). The development of such lines having low $\alpha$-linolenic acid/high linoleic acid can serve linseed as an edible oilseed crop, which can substantially substitute other edible oilseed crops. This can be certainly a significant contribution to Indian vegetable oil industry and also can be healthy oil to the society. Simultaneously, its seed meal can also serve as an ideal meal for poultry industry. So present study is undertaken with the objective of screening linseed lines having edible oil properties by combining ability analysis using line $\mathrm{x}$ tester mating design.

\section{Materials and Methods}

The present study was conducted in the field of Genetics and Plant Breeding, CSIRNational Botanical Research Institute, Lucknow situated $26^{\circ} 40^{\prime} \mathrm{E}$ longitudes and $80^{\circ} 45^{\prime}$ latitudes and at an altitude of $129 \mathrm{~m}$ above sea level. For the present study 30 
distinct lines and three testers were combined according to line $\mathrm{x}$ tester mating design, as a result $90 \mathrm{~F}_{1} \mathrm{~s}$ were developed. The seeds of $\mathrm{F}_{1}$ crosses were sown and selfed to get $90 \mathrm{~F}_{2}$. The final trial was conducted comprising of 33 parents, $90 \mathrm{~F}_{1} \mathrm{~s}$ and $90 \mathrm{~F}_{2} \mathrm{~s}$ with two rows of three replications. The rows were 3 meter in length and $30 \mathrm{~cm}$ apart. In each row plant to plant distance was maintained at $10 \mathrm{~cm}$ by thinning and recommended cultural practices and fertilizers were applied during whole crop season (Reddy and Pati, 1995).

The plants were selected randomly and the seeds of each plant were grinded and subjected to oil extraction through soxhlet method using hexane. The whole seeds were used for estimation of bulk density $\left(\mathrm{g} / \mathrm{cm}^{3}\right)$, true density $\left(\mathrm{g} / \mathrm{cm}^{3}\right)$ and porosity $(\%)$. The identification of colour of seeds were done using colour card of Royal Botanical society, Kew, England and the colour value of oil was determined using Lovibond Tintometer. The oil was taken separately for oil parameters analysis i.e. refractive index, specific gravity, acid value, linolenic acid (\%), linoleic acid $(\%)$ and iodine value. The remaining oil cakes were used for protein $(\%)$, free amino acids $(\mathrm{g} / \mathrm{Kg})$, carbohydrate $(\%)$ and phenol $(\%)$. The description of estimation procedure of different parameters are described below:

\section{Bulk density}

It is the mass of particles of the material divided by the total volume they occupy. The total volume includes particle volume, interparticle void volume and internal pore volume or is the ratio of mass sample of the seeds to its total volume and determined by filling a $1000 \mathrm{ml}$ measuring cylinder with seeds at height up to $15 \mathrm{~cm}$ and weighted.

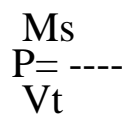

\section{True density}

It is the ratio of mass of the sample to its seed volume and was determined using water displacement method. $50 \mathrm{ml}$ of water was poured in a $100 \mathrm{ml}$ graduated measuring cylinder and $5 \mathrm{~g}$ seeds were immersed in that water. The amount of displaced water was recorded from the graduated scale of the cylinder. The ratio of weight of seeds to the volume of displaced water gave the true density.

\section{Porosity}

Porosity is the fraction of the space in bulk grain which is not occupied by the grain. The porosity of bulk seed was calculated from the values of true density and bulk density using the relationship as follows:

$\varepsilon=(\rho t-\rho b / \rho t) \times 100$

Where,

$\rho t=$ True density

$\rho b=$ Bulk density

\section{Protein}

The soluble protein of defatted powder was determined by using Bradford method. Sample (air-dried defatted powder $+800 \mu \mathrm{l}$ buffer extract) was placed in a test tube and centrifuged for $10 \mathrm{~min}$. The $5 \mu \mathrm{l}$ of sample was placed in ELISA plate and added 200 $\mu$ l of Bradford reagent. The buffer was prepared by adding 20mM HEPES (pH 8.0), glycerol 10\%, $1 \mathrm{mM}$ EDTA, $100 \mathrm{Mm}$ PMSF, $0.5 \mathrm{Mm}$ DTT and $1 \mathrm{mM}$ benzamidine. The absorbance of the sample was measured at $590 \mathrm{~nm}$ using spectrophotometer. Total protein was calculated as

Total protein content $(\%)=$

O.D. (s) - O.D. (b) x 18.3 x 100 
Where,

O.D. $(s)=$ OD of sample in buffer

O.D. (b) = OD of blank

18.3= Standard factor

\section{Free amino acid}

The total free amino acids of defatted powder were determined spectrophotometrically following the procedure of Hamilton and Van Slyke (1943). Sample (100mg defatted powder $+1.0 \mathrm{ml}$ buffer extract) was placed in a test tube and centrifuged. The supernatant was taken in test tube and added a mixture of $1.0 \mathrm{ml}$ ninhydrin and $1.0 \mathrm{ml}$ of pyridine solution. The test tube was heated for $30 \mathrm{~min}$ on a boiling water bath. The content of tubes was diluted with $50 \mathrm{ml}$ water and absorbance of the solution was measured at $570 \mathrm{~nm}$ using spectrophotometer. Total free amino acids were calculated as

Total free amino acids $(\mathrm{g} / \mathrm{kg})=$

Abs. of sample $\mathrm{x}$ Vol. of sample $\mathrm{x}$ Dilution factor

Weight of the sample

\section{Carbohydrate}

The total carbohydrate content of defatted powder was determined spectrophotometrically by Phenol-Sulphuric acid method. Air-dried defatted powder (100mg) was suspended in $5.0 \mathrm{ml}$ water with heating for 5 minutes and filtered through Whatmann filter paper. The residue was resuspended in 1\% HCL to remove all the traces of soluble sugar and filtered sample $(100 \mu \mathrm{l})$ was placed in a beaker. The sample was diluted to $900 \mu 1$ by de-ionized water and then added $1 \mathrm{ml}$ of $5 \%$ phenol solution and 5 mlsulphuric acid. The absorbance of each sample was monitored at $625 \mathrm{~nm}$ using spectrophotometer and calculated as
Carbohydrate $(\%)=$

$0.2857 \mathrm{x}$ Abs. of sample x Dilution factor x 100

Weight of seeds

\section{Phenolcontent}

$100 \mathrm{~g}$ seeds were extracted with ethanol $80 \%$ $(1000 \mathrm{ml})$ on shaker for 24 hours at room temperature. The extract was filtered and evaporated to dryness in a rotary evaporator to yield ethanolic extract (yield: 19.3\%). The Folin-Ciocalteu reagent $(0.2 \mathrm{ml}), \mathrm{H}_{2} \mathrm{O}(2 \mathrm{ml})$ and $15 \% \mathrm{Na}_{2} \mathrm{CO}_{3}$ solution $(1 \mathrm{ml})$ was mixed in the ethanolic extracts $(100 \mu \mathrm{l})$ and kept for 2 hours at room temperature. The absorbance of the mixture was then measured at $765 \mathrm{~nm}$ through spectrophotometer (Make: Shimadzu UV-1601). The mean of three readings was used and the total phenolic content was expressed as milligrams of gallic acid equivalents $/ 1 \mathrm{~g}$ extract. The coefficient of determination was $r^{2}=0.9958$ (Aslan et al., 2007).

\section{Oil percentage}

Total oil extracted from crushed seeds in culture tubes using soxhlet extraction method and calculated by using following formula:

Oil $\%=$

(Weight of culture tubes + weight of oil) Weight of culture tubes X 100

Weight of seeds

\section{Refractive index}

It was measured by Refractometer by measuring the speed of light in oil. The appropriate temperature correction factors were specified using the following equation:

$\mathrm{R}=\mathrm{R}^{\prime}+\mathrm{K}\left(\mathrm{T}^{\prime}-\mathrm{T}\right)$ 
Where,

$\mathrm{R}=$ Reading of the refractometer reduced to the specified temperature, $\mathrm{T}^{\circ} \mathrm{C}$.

$\mathrm{R}^{\prime}=$ reading at $\mathrm{T}^{\circ} \mathrm{C}$.

$\mathrm{K}=$ Constant 0.000385 for oils.

$T^{\prime}=$ temperature at which reading $\mathrm{R}^{\prime}$ is taken.

$\mathrm{T}=$ specified temperature.

\section{Specific gravity}

Specific gravity is the ratio of density (mass of a unit volume) of a substance to the density of a given reference material. Specific gravity usually means relative density with respect to water. The term "relative density" is often preferred in modern scientific usage. If relative density of a substance is less than one then it is less dense than the reference; if greater than one then it is denser than the reference.

Relative density $(\mathrm{RD})=\rho$ Substance $/ \rho$ Reference

Where,

$R D=$ Relative density

$\rho$ Substance $=$ Density of the substance being measured

$\rho$ Reference $=$ Density of the reference

Specific gravity was measured by using Pycnometer (specific gravity bottle). The empty bottle was weighted followed by filled bottle with water. Further the bottle was weighted filled with oil avoiding air bubbles, insert the stopper, holed it for few minutes and weight it. Finally, it was calculate by the formula

Specific gravity $=\mathrm{A}-\mathrm{B} / \mathrm{C}-\mathrm{B}$

Where,

$\mathrm{A}=$ Weight in $\mathrm{g}$ of the specific gravity bottle with oil.

$\mathrm{B}=$ Weight in $\mathrm{g}$ of the specific gravity bottle.

$\mathrm{C}=$ Weight in $\mathrm{g}$ of the specific gravity bottle with water.

\section{Acid value}

The acid value was done to estimate the total free fatty acids present in the sample. $2.5 \mathrm{~g}$ of appropriate sample of the material was dissolved in $20 \mathrm{ml}$ ethyl alcohol, previously neutilized by phenolphthalein with $\mathrm{KOH}$ solution. The sample was titrated with $\mathrm{KOH}$ $0.1 \mathrm{~N}$ solution until the solution remains faintly pink after $10 \mathrm{sec}$. of shaking. Finally, note the volume of $\mathrm{KOH}$ consumed and put that value in following formula

Acid Value $=56.10 \times \mathrm{V} \times \mathrm{N} /$ wt. of oil

Where,

$\mathrm{V}=$ Volume of $\mathrm{KOH}$ consumed

$\mathrm{N}=$ Normality of $\mathrm{KOH}$ solution

wt. $=$ Weight in $\mathrm{g}$ of material taken

\section{Fatty acid analysis}

The analysis was done using modified method of Schaffer and Holm (1950) and Indrayan et al., (2005).

\section{Linolenic acid}

It was calculated as per formula

Linolenic acid $(\mathrm{Y} \%)=$

$\alpha(268 \mathrm{~m} \mu) \times 100$

53.20

\section{Linoleic acid}

It was calculated as per formula

Linoleic acid $(\%)=$

$\alpha(234 \mathrm{~m} \mu)-(\mathrm{Y} / 100 \times 60.90) \times 100$

86.00 
Where,

53.20 is the specific absorption coefficient of pure linolenic acid at $268 \mathrm{~m} \mu, 60.90$ is the specific absorption coefficient of linolenic acid at $234 \mathrm{~m} \mu$ and 86.00 is the specific absorption coefficient of pure linoleic acid at $234 \mathrm{~m} \mu$.

\section{Iodine value}

The iodine value is iodine monochloride which is absorbed by sample to be tested and expressed in term of iodine. It is helpful to understand the concentration of unsaturated fatty acid present in testing sample and estimated by titration method using Wijs solution. It was calculated by the following formula

Iodine value $=$

(B-S) $\times 0.0126 \times 100$

Weight of sample

Where,

$\mathrm{B}=$ Blank test reading of burette

$\mathrm{S}=$ Titration of sample reading

$\mathrm{W}=$ Weight of the sample

\section{Statistical analysis}

The recorded data was subjected to combining ability analysis for selection of good general and specific combiners using INDOSTAT software, Hyderabad.

\section{Results and Discussion}

The estimates, magnitude and direction of general combining ability effects in both $F_{1}$ and $F_{2}$ generations of all 33 parents ( 3 testers and 30 lines) for all 14 traits are mentioned in table 1 and 3 . The negative values were desirable for the traits porosity, refractive index, specific gravity, acid value, linolenic acid and iodine value and positive values for rest of the traits. The range of gca effect for all the traits are mentioned in table 1. For oil percentage the only tester Neelam had significant maximum positive value in $F_{1}$ generation while none of the tester parent had significant positive value in $\mathrm{F}_{2}$ generation. Among lines parent Ex-313-23 followed by EC-110288 and EC-115161 in $F_{1}$ and parent CRISTA followed by Ex-5-36E and Ex-28-3 in $F_{2}$ generation had significant maximum positive values. For bulk density none of the tester had significant positive value in both generations. Among lines parent EC-41752 followed by EC-1041492 and Ex-3 had significant maximum positive value in $F_{1}$ while parent Ex-3 followed by Ezox Natural and Ex-339-6 had non-significant maximum value in $\mathrm{F}_{2}$ generation. For true density Heera had significant value for both generations. Among lines, parent EC-41752 followed by EC-41750 and EC-1041492 in $\mathrm{F}_{1}$ generation and parent FR-3 followed by parents EC99056, EC-41752, EC-104739, CRISTA, EC225125, ES-1474, ES-1531 and FRW-9 in $\mathrm{F}_{2}$ generation had significant positive high value. For Porosity Ex-7959 had high significant negative value in both generations. The line ES-14600 followed by EC-98994 and ES1474 in $F_{1}$ and line EC-115161 followed by EC-112689 and Ex-313-23 in $F_{2}$ had significant maximum negative value. For protein none of the tester had significant value in $F_{1}$ while Heera followed by Neelam had significant maximum positive value in $F_{2}$ generation. Among lines parent ES-1463 followed by EC-41752 and Ezox Natural in $\mathrm{F}_{1}$ and parent T-397 followed by ES-1496 and EC-110288 in $F_{2}$ had significant maximum positive value. Similarly for free amino none of the tester and line had significant positive value in both generations. The parent EC115162 followed by EC-112689, Mukta, EC115161, EC-115178 and EC-225125 in $\mathrm{F}_{1}$ and parent T-397 followed by ES-1496 and EC110288 in $F_{2}$ generation had maximum 
positive value. For carbohydrate Ex-7959 had highest significant maximum positive value in $F_{1}$ while Heera in $F_{2}$ generation. Among line, parents EC-115161 followed by ES-1463 and Ex-28-3 in $\mathrm{F}_{1}$ and parent EC-225125 followed by EC-99029 and EC-115186 in $\mathrm{F}_{2}$ generation had significant maximum positive value. For phenol Ex-7959 had significant maximum positive value in both generations. Among lines, parent Ex-5-36E followed by parent CRISTA and Ex-28-3 in $\mathrm{F}_{1}$ and parents Ex-5$36 \mathrm{E}$ followed by $\mathrm{T}-397$ and CRISTA in $\mathrm{F}_{2}$ generation had significant maximum positive values. For refractive index none of the tester and line had significant negative value in both generations. The parent EC-104739 in $\mathrm{F}_{1}$ and parent Ex-5-36E followed by FR-3 and CRISTA in $F_{2}$ generation had maximum negative value. For specific gravity none of tester had significant negative value. Among lines, parent FR-3 followed by Ezox Natural in $F_{1}$ and parent Ex-339-6 in $F_{2}$ generation had significant maximum negative value. Similarly for acid value none of the tester had significant negative value in $F_{1}$ generation while Heera had significant maximum negative value in $\mathrm{F}_{2}$ generation. Among lines, parent EC-104739 followed by ES-14600 in $\mathrm{F}_{1}$ and parent EC-112689 followed by EC104739 and $\mathrm{T}-397$ in $\mathrm{F}_{2}$ generation had significant maximum negative value. For linolenic acid tester Neelam had significant negative value in $\mathrm{F}_{2}$ generation. Among lines, the parent Ex-5-36E followed by CRISTA and EC-41750 in $F_{1}$ and parent EC-110288 followed by EC-98994 and Ex-5-36E in $F_{2}$ generation had significant maximum negative value. In linoleic Acid, Neelam had significant maximum positive value in both generations followed by Ex-7959 in $\mathrm{F}_{2}$ generation. Among lines, parent EC-112689 had significant maximum positive value followed by parents EC-104739 and FRW-9 in $\mathrm{F}_{1}$ and parent EC115178 followed by parents ES-1474 and EC112689 in $F_{2}$ generation. For iodine value Neelam had significant maximum negative value in both generations. Among lines, parent Ex-28-3 followed by CRISTA and Ex-5-36E in $\mathrm{F}_{1}$ and parent EC-110288 followed by EC98994 and Ex-5-36E in $F_{2}$ generation had significant maximum negative value.

The values of specific combining ability effects and their per se performance of 90 crosses for all 14 traits are presented in table 2 and 4 . For the traits viz. porosity, refractive index, specific gravity, acid value, linolenic acid and iodine value the negative values were considered desirable while for rest of the traits positive values were considered desirable. Based on per se performance five crosses possessed high value for three traits in $F_{1}$, six crosses for three traits and four crosses for four traits in $\mathrm{F}_{2}$ generation. The range of sca value for each trait are presented in table 2 . For oil percentage 29 crosses possessed significant positive values in $\mathrm{F}_{1}$ and 27 crosses in $F_{2}$ generation. The crosses having maximum significant positive values were Ex3 x Ex-7959, ES-1463 x Neelam and EC$110288 \mathrm{x}$ Neelam in $\mathrm{F}_{1}$ and EC-115162 $\mathrm{x}$ Neelam, EC-115161 x Ex-7959 and ES-1474 $x$ Heera in $F_{2}$ generation. For bulk density 34 crosses possessed significant positive values in $F_{1}$ and 47 crosses in $F_{2}$ generation. The cross ES-1463 x Neelam followed by crosses EC-115186 x Ex-7959 and EC-115178 x Heera in $F_{1}$ and cross T-397 $x$ Heera followed by crosses EC-115186 x Neelam and ES-1496 $x$ Ex-7959 in $F_{2}$ generation showed significant maximum positive values.

For true density 32 crosses in $F_{1}$ and 38 crosses in $\mathrm{F}_{2}$ generation had significant positive values. Three desirable crosses in descending order of magnitude were ES-1463 x Neelam, EC-115186 x Ex-7959 and Ex-313$23 \times$ Ex-7959 in $F_{1}$ and EC-115186 x Neelam, EC-110288 x Heera, EC-115178 x Heera, Ex$3 \times$ Heera and Ex-5-36E $x$ Heera in $F_{2}$ generation. Likewise for porosity 42 crosses in $F_{1}$ and 45 crosses in $F_{2}$ generation showed 
significant negative values. The cross EC115186 x Neelam followed by crosses Ex-28$3 \mathrm{x}$ Heera and EC-104739 $\mathrm{x}$ Ex-7959 in $\mathrm{F}_{1}$ generation and cross EC-41752 $\mathrm{x}$ Heera followed by Ex-28-3 x Neelam and Ex-3 x Ex7959 in $F_{2}$ generation had significant maximum negative values. For protein 28 crosses in $F_{1}$ and 45 crosses in $F_{2}$ generation had significant positive values. The cross Ezox Natural x Heera followed by crosses EC104739 x Heera and EC-1041492 x Ex-7959 in $F_{1}$ and cross ES-1531 x Neelam followed by crosses EC-115161 x Heera and EC-41752 $x$ Ex-7959 in $F_{2}$ generation had significant maximum positive values. For free amino acid 19 crosses had significant positive values in $F_{1}$ and 32 crosses in $F_{2}$ generation. Few desirable crosses in descending order of magnitude were EC-99056 x Ex-7959, FRW-9 x Heera, ES$1474 \times$ Neelam and EC-104739 x Neelam in $\mathrm{F}_{1}$ and EC-115161 $\mathrm{x}$ Heera, EC-98994 $\mathrm{x}$ Heera, EC-41752 x Ex-7959 and EC-99056 x Ex-7959 in $\mathrm{F}_{2}$ generation. For carbohydrate 44 crosses had significant positive values in $F_{1}$ and 43 crosses in $F_{2}$ generation. Based on the magnitude, cross ES-1531 x Heera followed by crosses FRW-9 x Heera and CRISTA $x$ Neelam in $F_{1}$ and cross EC-110288 x Neelam followed by crosses ES-1496 x Ex-7959 and FRW-9 $x$ Heera in $F_{2}$ generation exhibited significant maximum positive values. For phenol 42 crosses showed significant positive value in $F_{1}$ and 44 crosses in $F_{2}$ generation. The cross EC-115162 x Neelam followed by crosses EC-110288 x Heera and EC-112689 x Heera in $\mathrm{F}_{1}$ generation and cross EC-41750 x Ex-7959 followed by EC-110288 x Heera and EC-99056 x Ex-7959 in $F_{2}$ generation had significant maximum positive values. For refractive index only two crosses in $F_{1}$ and 22 crosses in $F_{2}$ generation had significant negative values. Based on the magnitude, cross EC-98994 x Neelam followed by cross EC-98994 $x$ Heera in $F_{1}$ and cross EC1126898 x Neelam followed by crosses ES$1474 \mathrm{x}$ Heera and EC-115178 x Heera in $\mathrm{F}_{2}$ generation exhibited maximum significant negative values. Similarly for specific gravity 18 crosses in $F_{1}$ and only two crosses in $F_{2}$ generation had significant negative values. Three desirable crosses in descending order of magnitude were EC-104739 x Neelam, Ex-5$36 \mathrm{E} \times$ Heera and EC-99029 $\mathrm{x}$ Ex-7959 in $\mathrm{F}_{1}$ and crosses EC-112689 x Ex-7959 and Ex$339-6$ x Neelam in $F_{2}$ generation. For acid value only one cross had significant negative value in $F_{1}$ and 43 crosses in $F_{2}$ generation. Based on magnitude, cross EC-98994 x Ex7959 in $F_{1}$ and cross ES-1463 $x$ Heera followed by crosses Ex-28-3 x Neelam and ES-1496 $x$ Neelam in $F_{2}$ generation had significant maximum negative values. For linolenic acid 27 crosses in $\mathrm{F}_{1}$ and 45 crosses in $\mathrm{F}_{2}$ generation possessed significant negative values. The cross EC-41750 x Ex-7959 followed by crosses EC-115186 x Ex-7959 and Ex-5-36E x Ex-7959 in $\mathrm{F}_{1}$ generation and cross T-397 x Neelam followed by crosses ES-14600 x Neelam and CRISTA x Heera in $\mathrm{F}_{2}$ generation possessed significant maximum negative values. Likewise for linoleic acid 44 crosses in $F_{1}$ and 42 crosses in $F_{2}$ generation had significant positive values. The cross Ex-3 x Ex-7959 followed by crosses Mukta x Heera and EC-115178 x Ex-7959 in $\mathrm{F}_{1}$ and Ezox Natural x Heera followed by crosses Ex-28-3 $x$ Ex-7969 and EC-1041492 x Neelam in $F_{2}$ generation had significant maximum positive values. For the iodine value 46 crosses in $F_{1}$ and 45 crosses in $F_{2}$ generation had significant negative values. The cross Ex-28-3 x Neelam followed by crosses Ezox Natural $x$ Heera and Mukta x Ex-7959 in $\mathrm{F}_{1}$ and cross EC-41750 x Ex-7959 followed by crosses ES-115186 x Ex-7959 and FRW-9 x Neelam in $F_{2}$ had significant maximum negative values.

Among the parents, seed colour varies between greyed orange (165A) or brown group (200D). The grading of colour was done using colour card of Royal Botanical Society, Kew. The texture of seeds and colour of oil of 
each parents are described in table 5. Among the $F_{1}$ crosses, seed colour vary between greyed orange (165A/166A) or brown group (200D) while seeds of majority of crosses in $\mathrm{F}_{2}$ belonged with brown group (200D) except few ones having seed colour of greyed orange group (165A). The texture of seeds and colour of oil of each crosses in both generations are described in table 6 and 7.

There is always a need to improve the oil yield and its edible characteristic in secondary oilseed crops. The yield is a polygenic controlled trait and depends on large number of other related traits. Selection on the basis of yield alone is usually not effective, whereas selection along with its component characters could be more effective and reliable. There are several instances the importance of both additive and non-additive types of gene action for yield components was reported (Mistry et al., 2016 and Devi et al., 2018).

Linseed oil has less shelf life due to its rapid oxidation property (Kozlowska et al., 2008; Singh et al., 2011 and Faintuch et al., 2011). Despite this, its physical and chemical properties is nearby similar to existing oilseed crops which proven the use of linseed oil for edible purposes. Various edible forms of flax are available in the food market as whole flaxseeds, milled flax, roasted flax etc. Linseed seeds are found in two colours (1) brown; and (2) yellow or golden and both have similar nutritional characteristics (Morris, 2007; Gonarkar and Jain, 2013 and Kaur et al., 2018). It is evident by the literature that seed colour is also helpful to get an idea for fatty acid profile that can also be used as morphological marker.

Yield is a quantitative trait which is pretentious by genotype $\times$ environment $(G \times$ E) hence combining ability would depend on the set of germplasm and environment where they are tested. The selection of best performing lines is main objective in the crop breeding programme (Fasahat et al., 2016 and Patel and Patidar, 2018). Combining ability is defined as ability of parents to combine in hybridization process to transfer the desirable genes or characters to their progenies. Two concepts of combining ability i.e. general combining ability (GCA) and specific combining ability (SCA) have had important influence on inbred line evaluation and population development in crop breeding. GCA is an effective tool used in selection of parents based on performance of their progenies, usually in the $F_{1}$ but it has also been used in $\mathrm{F}_{2}$ and later generations (Fn). The high GCA estimate indicates higher heritability and less environmental effects. Additive variances are fixable in nature while non-additive are non-fixable and due to dominance and epistatic gene action. The dominance variances diminish by half with each generation of selfing and thus unexploitable in pure lines. Epistatic variance also declines on selfing. In GCA determination, SCA usually acts as a masking effect. By selecting genetically broad testers or increasing number of testers, SCA impact can be decreases. Parental choice only on the basis of SCA effect has limited value in breeding programs. Therefore, SCA effect should be used in combination with a high performance per se hybrid, favourable SCA estimates, and involving at least one parent with high GCA (Fasahat et al., 2016). Observations of performance of different cross patterns on the basis of SCA have been used to get result on gene action perform. High SCA effects in crosses where both parents are good general combiners (i.e., good GCA $\times$ good GCA) may be due to additive $x$ additive gene action. The high SCA effects derived from crosses including good $\times$ poor general combiner parents may be credited to favourable additive effects of the good general combiner parent and epistatic effects of poor general combiner, which fulfils the favourable plant trait (Kumar et al., 2011). 
Table.1 General combining ability effects in $\mathrm{F}_{1}$ and $\mathrm{F}_{2}$ generations for different traits

\begin{tabular}{|c|c|c|c|c|c|c|c|c|c|c|c|}
\hline \multirow[t]{2}{*}{ S.No. } & \multirow[t]{2}{*}{ Parents } & \multicolumn{2}{|l|}{ OIL } & \multicolumn{2}{|l|}{ BD } & \multicolumn{2}{|l|}{ TD } & \multicolumn{2}{|l|}{ PO } & \multicolumn{2}{|l|}{ PR } \\
\hline & & $\mathrm{F}_{1}$ & $\mathrm{~F}_{2}$ & $\mathrm{~F}_{1}$ & $F_{1}$ & $\mathrm{~F}_{1}$ & $\mathrm{~F}_{2}$ & $\mathrm{~F}_{1}$ & $F_{2}$ & $F_{1}$ & $\mathrm{~F}_{2}$ \\
\hline \multicolumn{12}{|c|}{ Testers } \\
\hline 1 & Heera & -1.251 & -0.594 & 0.056 & -0.010 & $0.079 *$ & $0.644 * *$ & $0.671 * *$ & $3.409 * *$ & 0.345 & $0.519 * *$ \\
\hline 2 & Ex-7959 & -0.469 & 0.573 & -0.006 & 0.002 & -0.017 & $-0.667 * *$ & $-0.811 * *$ & $-3.724 * *$ & -0.555 & $-0.959 * *$ \\
\hline \multirow[t]{2}{*}{3} & Neelam & $1.721 *$ & 0.022 & -0.050 & 0.008 & -0.062 & $0.022 *$ & 0.139 & $0.315 * *$ & 0.210 & $0.440 * *$ \\
\hline & gi-gj (tester) & 0.337 & 0.450 & 0.015 & 0.026 & 0.015 & 0.005 & 0.044 & 0.005 & 0.146 & 0.005 \\
\hline \multicolumn{12}{|c|}{ Lines } \\
\hline 1 & EC-41750 & $-7.098 * *$ & 0.493 & $0.109 *$ & -0.112 & $0.431 * *$ & $0.078 * *$ & $-3.738 * *$ & $0.680 * *$ & $2.228 * *$ & $-6.945 * *$ \\
\hline 2 & EC-41752 & -1.739 & $4.000 *$ & $0.579 * *$ & -0.057 & $0.568 * *$ & $1.411 * *$ & $-1.019 * *$ & $8.164 * *$ & $3.155 * *$ & $-0.133 * *$ \\
\hline 3 & EC-98994 & $-4.215 * *$ & 0.650 & 0.059 & 0.001 & 0.048 & $-0.256 * *$ & $-10.349 * *$ & $-0.453 * *$ & $1.618 * *$ & $1.117 * *$ \\
\hline 4 & T-397 & $-5.577 * *$ & $-3.267 *$ & $-0.265 * *$ & -0.004 & $-0.276 * *$ & $0.078 * *$ & $17.123 * *$ & $-0.401 * *$ & -0.890 & $7.129 * *$ \\
\hline 5 & EC-99029 & -0.887 & 1.973 & $-0.200 * *$ & 0.034 & $-0.212 * *$ & $-0.811 * *$ & $-6.908 * *$ & $-4.789 * *$ & $-2.101 * *$ & $-0.640 * *$ \\
\hline 6 & EC-99056 & -0.027 & $-6.305 * *$ & -0.083 & 0.045 & -0.094 & $1.411 * *$ & $-0.494 * *$ & $7.335 * *$ & $1.203 *$ & $-4.388 * *$ \\
\hline 7 & EC-104739 & 2.032 & $-8.279 * *$ & $-0.629 * *$ & 0.017 & $-0.640 * *$ & $1.411 * *$ & $-1.567 * *$ & $7.589 * *$ & 0.042 & $-0.269 * *$ \\
\hline 8 & CRISTA & $-2.392 *$ & $11.540 * *$ & $-0.262 * *$ & -0.125 & $-0.273 * *$ & $1.411 * *$ & $0.718 * *$ & $8.926 * *$ & $-1.012 *$ & $-3.100 * *$ \\
\hline 9 & EC-110288 & $8.696 * *$ & $-8.683 * *$ & $-0.163 * *$ & -0.020 & $-0.174 * *$ & $-0.533 * *$ & $-0.364 *$ & $-4.282 * *$ & $0.993 *$ & $4.357 * *$ \\
\hline 10 & EC-112689 & $5.895 * *$ & $3.201 *$ & 0.071 & 0.013 & 0.060 & $-1.367 * *$ & $-3.285 * *$ & $-8.647 * *$ & 0.325 & $0.892 * *$ \\
\hline 11 & EC-115161 & $8.207 * *$ & -1.559 & 0.095 & 0.012 & 0.084 & $-1.367 * *$ & $-3.647 * *$ & $-8.720 * *$ & $-1.481 * *$ & $-0.728 * *$ \\
\hline 12 & EC-115162 & $3.558 * *$ & 1.140 & $-0.416 * *$ & -0.055 & $-0.428 * *$ & $-0.256 * *$ & $2.596 * *$ & $0.113 * *$ & -0.802 & $1.888 * *$ \\
\hline 13 & EC-115178 & $8.154 * *$ & $-8.684 * *$ & $-0.488 * *$ & 0.027 & $-0.499 * *$ & 0.022 & $9.445 * *$ & $-0.477 * *$ & -0.588 & $-0.094 * *$ \\
\hline 14 & EC-115186 & $2.689 *$ & 0.935 & $-0.471 * *$ & 0.024 & $-0.482 * *$ & 0.022 & $3.934 * *$ & $-0.387 * *$ & $-1.652 * *$ & $-1.450 * *$ \\
\hline 15 & EC-225125 & $-7.229 * *$ & 0.320 & $0.234 * *$ & 0.045 & $0.223 * *$ & $0.578 * *$ & $-5.203 * *$ & $3.167 * *$ & $-5.551 * *$ & $-1.275 * *$ \\
\hline 16 & EC-1041492 & $-7.174 * *$ & -1.345 & $0.400 * *$ & $-0.172 *$ & $0.389 * *$ & $-0.256 * *$ & $1.276 * *$ & $5.177 * *$ & $-2.225 * *$ & $0.668 * *$ \\
\hline 17 & Mukta & $5.048 * *$ & 2.428 & 0.049 & 0.010 & 0.038 & $-0.811 * *$ & $-4.720 * *$ & $-4.578 * *$ & $-1.217 *$ & $-1.685 * *$ \\
\hline 18 & ES-1463 & 0.014 & $-6.915 * *$ & $0.103 *$ & $-0.264 * *$ & 0.092 & $-0.811 * *$ & $-4.655 * *$ & $-1.584 * *$ & $4.975 * *$ & $0.882 * *$ \\
\hline 19 & ES-1474 & $-4.718 * *$ & $6.165 *$ & $-0.106 *$ & 0.086 & $-0.117 *$ & $0.578 * *$ & $-7.863 * *$ & $2.878 * *$ & $2.965 * *$ & $-1.538 * *$ \\
\hline 20 & ES-1496 & $2.672 *$ & $-5.137 * *$ & $-0.139 * *$ & -0.007 & $-0.151 * *$ & $-0.256 * *$ & $-0.806 * *$ & $-0.370 * *$ & 0.910 & $5.099 * *$ \\
\hline 21 & ES-1531 & $-11.649 * *$ & $-4.860 * *$ & $0.102 * *$ & -0.038 & 0.091 & $0.578 * *$ & $4.851 * *$ & $4.000 * *$ & $-1.364 * *$ & $-1.021 * *$ \\
\hline 22 & ES-14600 & $3.136 * *$ & $-7.745 * *$ & $-0.128 *$ & 0.060 & $-0.139 * *$ & $-0.811 * *$ & $-12.562 * *$ & $-5.051 * *$ & $1.779 * *$ & $3.108 * *$ \\
\hline 23 & Ex-3 & $2.548 *$ & -2.109 & $0.399 * *$ & 0.166 & $0.388 * *$ & 0.022 & $-7.242 * *$ & $-2.089 * *$ & 0.662 & $-1.099 * *$ \\
\hline 24 & Ex-5-36E & $-2.649 *$ & $11.255 * *$ & $0.191 * *$ & -0.040 & $0.180 * *$ & 0.022 & $3.542 * *$ & $0.114 * *$ & $-5.165 * *$ & $3.332 * *$ \\
\hline 25 & Ex-28-3 & -0.494 & $11.091 * *$ & $0.253 * *$ & 0.097 & $0.242 * *$ & $-0.811 * *$ & $2.707 * *$ & $-5.621 * *$ & -0.256 & $-0.142 * *$ \\
\hline 26 & Ex-313-23 & $10.034 * *$ & -0.462 & $0.233 * *$ & 0.007 & $0.222 * *$ & $-1.367 * *$ & $-5.280 * *$ & $-8.547 * *$ & -0.666 & $-4.066 * *$ \\
\hline 27 & Ex-339-6 & -0.836 & 1.355 & $0.111 *$ & 0.137 & $0.100 *$ & $-0.478 * *$ & $-3.768 * *$ & $-5.944 * *$ & 0.274 & $0.267 * *$ \\
\hline 28 & Ezox Natural & $-4.215 * *$ & $3.835 *$ & 0.073 & 0.147 & 0.062 & $-0.256 * *$ & $12.392 * *$ & $-1.907 * *$ & $2.989 * *$ & $-1.167 * *$ \\
\hline 29 & FR-3 & -0.550 & 0.635 & 0.095 & -0.027 & 0.084 & $2.244 * *$ & $13.199 * *$ & $12.003 * *$ & $-1.281 * *$ & $-0.455 * *$ \\
\hline \multirow[t]{2}{*}{30} & FRW-9 & -1.233 & $4.338 *$ & $0.193 * *$ & -0.007 & $0.182 * *$ & $0.578 * *$ & $11.689 * *$ & $3.704 * *$ & $2.130 * *$ & $1.458 * *$ \\
\hline & gi-gj (line) & 1.066 & 1.421 & 0.047 & 0.081 & 0.047 & 0.050 & 0.141 & 0.015 & 0.462 & 0.014 \\
\hline
\end{tabular}


Table.1 Continued

\begin{tabular}{|c|c|c|c|c|c|c|c|c|c|c|c|}
\hline \multirow[t]{2}{*}{ S.No. } & \multirow[t]{2}{*}{ Parents } & \multicolumn{2}{|l|}{ FAA } & \multicolumn{2}{|l|}{ CAR } & \multicolumn{2}{|l|}{ PHE } & \multicolumn{2}{|l|}{ RF } & \multicolumn{2}{|l|}{ SG } \\
\hline & & $\mathrm{F}_{1}$ & $\mathrm{~F}_{2}$ & $\mathrm{~F}_{1}$ & $\mathrm{~F}_{1}$ & $\mathrm{~F}_{1}$ & $\mathrm{~F}_{2}$ & $F_{1}$ & $\mathrm{~F}_{2}$ & $F_{1}$ & $\mathrm{~F}_{2}$ \\
\hline \multicolumn{12}{|c|}{ Testers } \\
\hline 1 & Heera & 0.001 & 0.001 & $-0.617 * *$ & $0.296 * *$ & $-0.138 * *$ & $-0.184 * *$ & 0.001 & 0.001 & 0.001 & 0.005 \\
\hline 2 & Ex-7959 & -0.001 & -0.001 & $0.768 * *$ & $-0.228 * *$ & $0.101 * *$ & $0.390 * *$ & 0.001 & 0.004 & -0.002 & -0.004 \\
\hline 3 & Neelam & 0.001 & 0.001 & $-0.151 * *$ & $-0.069 * *$ & $0.037 * *$ & $-0.206 * *$ & -0.001 & -0.004 & 0.001 & -0.002 \\
\hline & gi-gj (tester) & 0.004 & 0.005 & 0.005 & 0.005 & 0.005 & 0.005 & 0.005 & 0.005 & 0.003 & 0.012 \\
\hline \multicolumn{12}{|c|}{ Lines } \\
\hline 1 & EC-41750 & 0.003 & -0.006 & $-1.496 * *$ & $-5.571 * *$ & $-0.599 * *$ & $0.750 * *$ & 0.001 & 0.013 & 0.009 & 0.008 \\
\hline 2 & EC-41752 & 0.003 & 0.000 & $-6.807 * *$ & $3.190 * *$ & $0.495 * *$ & $-0.275 * *$ & 0.001 & $0.030 *$ & 0.010 & -0.003 \\
\hline 3 & EC-98994 & -0.001 & 0.001 & $-3.774 * *$ & $-3.343 * *$ & $-0.647 * *$ & $0.340 * *$ & 0.027 & 0.015 & 0.005 & -0.015 \\
\hline 4 & T-397 & -0.003 & 0.007 & $-5.267 * *$ & $-0.143 * *$ & $-0.096 * *$ & $1.349 * *$ & 0.000 & 0.020 & -0.005 & 0.007 \\
\hline 5 & EC-99029 & -0.001 & -0.001 & 0.027 & $9.190 * *$ & $-0.595 * *$ & $0.344 * *$ & 0.001 & 0.018 & -0.013 & -0.016 \\
\hline 6 & EC-99056 & -0.003 & -0.004 & $-1.430 * *$ & $6.466 * *$ & $1.092 * *$ & $0.397 * *$ & -0.003 & 0.023 & 0.011 & 0.036 \\
\hline 7 & EC-104739 & -0.002 & 0.000 & $5.126 * *$ & $-8.257 * *$ & $-1.520 * *$ & $-1.129 * *$ & -0.010 & 0.004 & -0.001 & 0.033 \\
\hline 8 & CRISTA & -0.001 & -0.003 & $-1.040 * *$ & $-2.467 * *$ & $1.410 * *$ & $0.937 * *$ & -0.003 & -0.021 & -0.003 & -0.007 \\
\hline 9 & EC-110288 & -0.001 & 0.004 & $-2.828 * *$ & $-1.400 * *$ & $0.394 * *$ & $-0.085 * *$ & -0.002 & 0.029 & 0.006 & 0.022 \\
\hline 10 & EC-112689 & 0.005 & 0.001 & $-2.716 * *$ & $-4.105 * *$ & $-0.683 * *$ & $-0.652 * *$ & -0.004 & -0.014 & 0.006 & -0.071 \\
\hline 11 & EC-115161 & 0.004 & -0.001 & $7.426 * *$ & $0.067 * *$ & $0.037 * *$ & $-0.621 * *$ & -0.002 & 0.003 & 0.013 & 0.011 \\
\hline 12 & EC-115162 & 0.006 & 0.002 & $5.420 * *$ & $-1.752 * *$ & $-0.767 * *$ & $-1.506 * *$ & -0.003 & 0.003 & 0.012 & 0.023 \\
\hline 13 & EC-115178 & 0.004 & 0.000 & $2.801 * *$ & $-1.248 * *$ & $-0.398 * *$ & $-0.906 * *$ & -0.005 & 0.018 & 0.013 & 0.068 \\
\hline 14 & EC-115186 & 0.001 & -0.002 & $-3.447 * *$ & $6.543 * *$ & $-0.844 * *$ & $0.314 * *$ & -0.003 & 0.003 & 0.010 & -0.026 \\
\hline 15 & EC-225125 & 0.004 & -0.001 & $4.829 * *$ & $11.914 * *$ & $-0.478 * *$ & $0.166 * *$ & -0.003 & -0.005 & -0.008 & -0.024 \\
\hline 16 & EC-1041492 & -0.003 & 0.001 & $-1.701 * *$ & $2.276 * *$ & -0.009 & $0.554 * *$ & 0.002 & 0.005 & 0.001 & 0.034 \\
\hline 17 & Mukta & 0.005 & -0.002 & $-2.319 * *$ & $-0.829 * *$ & $-1.336 * *$ & $-0.180 * *$ & -0.001 & -0.002 & 0.014 & -0.043 \\
\hline 18 & ES-1463 & 0.000 & 0.001 & $7.159 * *$ & $-6.066 * *$ & $0.486 * *$ & $-0.289 * *$ & -0.001 & 0.004 & 0.012 & 0.032 \\
\hline 19 & ES-1474 & 0.002 & -0.002 & $3.109 * *$ & $0.305 * *$ & $-0.814 * *$ & $-0.854 * *$ & 0.000 & -0.014 & 0.011 & -0.023 \\
\hline 20 & ES-1496 & 0.003 & 0.005 & $-2.138 * *$ & $2.228 * *$ & $-0.207 * *$ & $-0.095 * *$ & 0.000 & 0.005 & 0.000 & 0.007 \\
\hline 21 & ES-1531 & -0.001 & -0.001 & $0.553 * *$ & $0.419 * *$ & $-0.643 * *$ & $-0.981 * *$ & 0.000 & -0.016 & -0.015 & 0.058 \\
\hline 22 & ES-14600 & -0.002 & 0.003 & $0.350 * *$ & $0.571 * *$ & $-0.449 * *$ & $-0.845 * *$ & 0.000 & -0.010 & -0.003 & 0.032 \\
\hline 23 & Ex-3 & -0.002 & -0.001 & $-5.412 * *$ & $-1.657 * *$ & $0.464 * *$ & $0.141 * *$ & 0.001 & -0.015 & -0.001 & 0.024 \\
\hline 24 & Ex-5-36E & -0.002 & 0.003 & $4.431 * *$ & $-3.524 * *$ & $2.027 * *$ & $1.387 * *$ & 0.000 & -0.024 & 0.006 & -0.009 \\
\hline 25 & Ex-28-3 & -0.002 & 0.000 & $5.876 * *$ & $-3.905 * *$ & $1.405 * *$ & $0.474 * *$ & 0.001 & -0.012 & 0.001 & -0.027 \\
\hline 26 & Ex-313-23 & -0.005 & -0.004 & $-0.162 * *$ & $-8.495 * *$ & $-0.206 * *$ & $-0.036 * *$ & 0.000 & -0.004 & 0.003 & -0.011 \\
\hline 27 & Ex-339-6 & -0.004 & 0.000 & $-4.537 * *$ & $2.171 * *$ & $0.867 * *$ & $0.514 * *$ & 0.000 & -0.008 & -0.016 & $-0.112 * *$ \\
\hline 28 & Ezox Natural & -0.003 & -0.001 & $-0.236 * *$ & $4.390 * *$ & $0.732 * *$ & $0.075 * *$ & 0.001 & -0.006 & $-0.035 * *$ & -0.022 \\
\hline 29 & FR-3 & 0.000 & 0.001 & $1.308 * *$ & $0.457 * *$ & $0.328 * *$ & $0.462 * *$ & 0.001 & -0.023 & $-0.040 * *$ & 0.007 \\
\hline \multirow[t]{2}{*}{30} & FRW-9 & $-0.002 * *$ & 0.001 & $-3.104 * *$ & $2.571 * *$ & $0.553 * *$ & $0.249 * *$ & 0.000 & -0.018 & -0.007 & 0.007 \\
\hline & gi-gj (line) & 0.015 & 0.015 & 0.015 & 0.014 & 0.015 & 0.003 & 0.015 & 0.015 & 0.011 & 0.065 \\
\hline
\end{tabular}


Table.1 Continued

\begin{tabular}{|c|c|c|c|c|c|c|c|c|c|}
\hline \multirow[t]{2}{*}{ S. No. } & \multirow[t]{2}{*}{ Parents } & \multicolumn{2}{|l|}{ AV } & \multicolumn{2}{|l|}{$\mathbf{L N}$} & \multicolumn{2}{|l|}{$\mathbf{L L}$} & \multicolumn{2}{|l|}{ IV } \\
\hline & & $F_{1}$ & $\mathrm{~F}_{2}$ & $F_{1}$ & $F_{1}$ & $\mathrm{~F}_{2}$ & $\mathrm{~F}_{2}$ & $\mathrm{~F}_{2}$ & $\mathrm{~F}_{2}$ \\
\hline \multicolumn{10}{|c|}{ Testers } \\
\hline 1 & Heera & -0.092 & $-0.074 * *$ & -0.848 & $1.038 * *$ & $-0.397 * *$ & $-1.208 * *$ & $1.965 * *$ & $0.755 * *$ \\
\hline 2 & Ex-7959 & 0.034 & $0.031^{*}$ & 0.092 & $0.480^{* *}$ & $-0.638 * *$ & $0.286 * *$ & $1.831 * *$ & $-0.210 * *$ \\
\hline 3 & Neelam & 0.058 & $0.043^{*}$ & 0.756 & $-1.519 * *$ & $1.036 * *$ & $0.922 * *$ & $-3.796 * *$ & $-0.546 * *$ \\
\hline & gi-gj (tester) & 0.029 & 0.007 & 0.347 & 0.003 & 0.023 & 0.077 & 0.083 & 0.020 \\
\hline \multicolumn{10}{|c|}{ Lines } \\
\hline 1 & EC-41750 & -0.171 & $-0.093 * *$ & $-6.889 * *$ & $12.912 * *$ & $-3.135 * *$ & $3.171 * *$ & $-1.522 * *$ & $-1.088 * *$ \\
\hline 2 & EC-41752 & -0.038 & $0.064 * *$ & $-2.993 *$ & $-1.895^{* *}$ & $-0.188 *$ & $-4.566 * *$ & $3.581 * *$ & $0.573 * *$ \\
\hline 3 & EC-98994 & 0.163 & $0.044^{*}$ & 1.845 & $-12.033^{* *}$ & $-0.425 * *$ & $-7.682 * *$ & $0.399 *$ & $-6.958 * *$ \\
\hline 4 & T-397 & 0.049 & $-0.397 * *$ & -1.503 & $-2.427 * *$ & $1.550 * *$ & $3.637 * *$ & $2.138 * *$ & $-0.760 * *$ \\
\hline 5 & EC-99029 & 0.129 & $0.182 * *$ & $-3.141 * *$ & $1.120^{* *}$ & $-2.939 * *$ & $0.734 * *$ & $-2.839 * *$ & $-4.465 * *$ \\
\hline 6 & EC-99056 & -0.031 & $0.152 * *$ & 1.874 & $5.175^{* *}$ & $4.695 * *$ & $2.356 * *$ & $8.264 * *$ & $3.204 * *$ \\
\hline 7 & EC-104739 & $0.206^{* *}$ & $-0.400 * *$ & $3.881 * *$ & $9.745^{* *}$ & $6.195 * *$ & $6.497 * *$ & $7.551 * *$ & $0.205 * *$ \\
\hline 8 & CRISTA & 0.085 & $-0.145^{* *}$ & $-10.854 * *$ & $-3.240 * *$ & $-7.633 * *$ & $-3.419 * *$ & $-6.817 * *$ & $2.544 * *$ \\
\hline 9 & EC-110288 & -0.010 & $-0.201 * *$ & 0.177 & $-15.219 * *$ & $2.332 * *$ & $-12.240 * *$ & $-1.024 * *$ & $-7.641 * *$ \\
\hline 10 & EC-112689 & -0.050 & $-0.507 * *$ & $3.658 * *$ & $-4.804 * *$ & $6.544 * *$ & $6.577 * *$ & $5.551 * *$ & $-1.577 * *$ \\
\hline 11 & EC-115161 & 0.013 & $-0.132 * *$ & 1.264 & $2.743 * *$ & $3.161 * *$ & $-2.457 * *$ & $4.304 * *$ & $4.806 * *$ \\
\hline 12 & EC-115162 & 0.034 & $-0.113 * *$ & $4.139 * *$ & $8.836^{* *}$ & $4.901 * *$ & $3.452 * *$ & $7.514 * *$ & $8.299 * *$ \\
\hline 13 & EC-115178 & -0.065 & $0.165^{* *}$ & -0.880 & $3.874 * *$ & $-4.372 * *$ & $6.743 * *$ & $2.297 * *$ & $5.161 * *$ \\
\hline 14 & EC-115186 & -0.103 & $-0.209 * *$ & $-3.036 * *$ & $2.164 * *$ & $0.307 * *$ & $-1.251 * *$ & $-0.449 *$ & $-2.477 * *$ \\
\hline 15 & EC-225125 & $0.222 * *$ & $0.120^{* *}$ & $4.541 * *$ & $3.716^{* *}$ & $1.409 * *$ & -0.073 & $4.981 * *$ & $7.538 * *$ \\
\hline 16 & EC-1041492 & 0.185 & $0.469 * *$ & $-2.765 * *$ & $-1.257 * *$ & $2.600 * *$ & $2.373 * *$ & $6.070 * *$ & $-3.857 * *$ \\
\hline 17 & Mukta & 0.052 & $-0.223 * *$ & -2.034 & $-1.813^{* *}$ & $2.947 * *$ & $-3.171 * *$ & $10.124 * *$ & $1.861 * *$ \\
\hline 18 & ES-1463 & 0.068 & $0.838 * *$ & $3.597 * *$ & $-1.633^{* *}$ & $-1.244 * *$ & $2.461 * *$ & $4.468 * *$ & $-1.465 * *$ \\
\hline 19 & ES-1474 & -0.060 & $-0.207 * *$ & 0.777 & $3.954 * *$ & $-3.190 * *$ & $6.639 * *$ & $-3.199 * *$ & $0.866 * *$ \\
\hline 20 & ES-1496 & 0.054 & $0.534 * *$ & $-3.139 * *$ & $-1.646 * *$ & $-4.253 * *$ & $-4.204 * *$ & $-5.358 * *$ & $5.483 * *$ \\
\hline 21 & ES-1531 & -0.005 & $-0.092 * *$ & 2.215 & $7.266^{* *}$ & $-2.116 * *$ & $2.971 * *$ & $0.975 * *$ & $-2.515 * *$ \\
\hline 22 & ES-14600 & $-0.194 *$ & 0.040 & $2.610 *$ & $-3.646^{* *}$ & $1.730 * *$ & $-7.704 * *$ & $7.434 * *$ & $-4.930 * *$ \\
\hline 23 & Ex-3 & -0.099 & $-0.072 * *$ & -1.252 & $5.090 * *$ & $-2.662 * *$ & $-5.348 * *$ & $4.370 * *$ & $1.156 * *$ \\
\hline 24 & Ex-5-36E & -0.088 & $0.209^{* *}$ & $-12.655 * *$ & $-10.143 * *$ & $-5.013 * *$ & $-7.282 * *$ & $-6.326 * *$ & $-6.148 * *$ \\
\hline 25 & Ex-28-3 & -0.089 & $0.101 * *$ & $7.337 * *$ & $-6.293^{* *}$ & $-0.150 * *$ & $4.973 * *$ & $-9.419 * *$ & $-3.494 * *$ \\
\hline 26 & Ex-313-23 & -0.019 & 0.014 & $4.306 * *$ & $0.457 * *$ & $-3.223 * *$ & $-0.472 *$ & $6.674 * *$ & $-0.146 *$ \\
\hline 27 & Ex-339-6 & 0.011 & $0.486^{* *}$ & $4.067 * *$ & $6.684 * *$ & $-1.063 * *$ & $4.429 * *$ & $6.230 * *$ & $7.873 * *$ \\
\hline 28 & Ezox Natural & $0.265 * *$ & $-0.115^{* *}$ & 0.478 & $-9.134 * *$ & $-1.935 * *$ & $-3.950 * *$ & $2.101 * *$ & $-5.135 * *$ \\
\hline 29 & FR-3 & -0.065 & $-0.166 * *$ & $4.286 * *$ & $-0.828 * *$ & 0.0700 & $2.645 * *$ & $-4.376 * *$ & $-0.462 * *$ \\
\hline \multirow[t]{2}{*}{30} & FRW-9 & -0.037 & $-0.347 * *$ & 0.087 & $2.272 * *$ & $5.100 * *$ & $4.163 * *$ & $-3.696 * *$ & $3.545 * *$ \\
\hline & gi-gj (line) & 0.094 & 0.021 & 1.098 & 0.011 & 0.074 & 0.244 & 0.262 & 0.066 \\
\hline
\end{tabular}

$\mathrm{OIL}=$ Oil $(\%), \mathrm{BD}=$ Bulk density $\left(\mathrm{g} / \mathrm{cm}^{3}\right), \mathrm{TD}=$ True density $\left(\mathrm{g} / \mathrm{cm}^{3}\right), \mathrm{PO}=$ Porosity $(\%), \mathrm{PR}=$ Protein $(\%), \mathrm{FAA}=$ Free amino acids $(\mathrm{g} / \mathrm{Kg}), \mathrm{CAR}=$ Carbohydrate $(\%), \mathrm{PHE}=\mathrm{Phenol}(\%), \mathrm{RF}=$ Refractive index, $\mathrm{SG}=$ Specific gravity, AV= Acid value, $\mathrm{LN}=$ Linolenic acid (\%), LL= Linoleic acid (\%) and IV= Iodine value. 
Int.J.Curr.Microbiol.App.Sci (2019) 8(1): 1118-1148

Table.2 Specific combining ability effects in $\mathrm{F}_{1}$ and $\mathrm{F}_{2}$ generations for different traits in linseed

\begin{tabular}{|c|c|c|c|c|c|c|c|c|c|c|c|}
\hline \multirow[t]{2}{*}{ S.No } & \multirow[t]{2}{*}{ Crosses } & \multicolumn{2}{|c|}{ OIL } & \multicolumn{2}{|l|}{$\mathrm{BD}$} & \multicolumn{2}{|l|}{ TD } & \multicolumn{2}{|l|}{$\mathrm{PO}$} & \multicolumn{2}{|l|}{ PR } \\
\hline & & $\mathrm{F}_{1}$ & $\mathrm{~F}_{2}$ & $\mathrm{~F}_{1}$ & $\mathrm{~F}_{2}$ & $\mathrm{~F}_{1}$ & $\mathrm{~F}_{2}$ & $\mathrm{~F}_{1}$ & $\mathrm{~F}_{2}$ & $\mathrm{~F}_{1}$ & $\mathrm{~F}_{2}$ \\
\hline 1 & EC-41750 x Heera & $4.344^{*}$ & $9.754 * *$ & -0.107 & $-0.047 * *$ & $0.538^{* *}$ & 0.022 & -0.053 & $-2.838 * *$ & 0.089 & $5.249 * *$ \\
\hline 2 & EC-41750 x Ex-7959 & $4.018^{*}$ & -2.313 & $-0.328 * *$ & $0.314 * *$ & $-0.651 * *$ & $0.333 * *$ & $4.763 * *$ & $0.568 * *$ & -1.353 & $1.106^{* *}$ \\
\hline 3 & EC-41750 x Neelam & $-8.362 * *$ & $-7.442 * *$ & $0.435^{* *}$ & $-0.267 * *$ & 0.113 & $-0.356^{* *}$ & $-4.711 * *$ & $2.269 * *$ & 1.263 & $-6.355^{* *}$ \\
\hline 4 & EC-41752 x Heera & $-7.980 * *$ & $-7.252 * *$ & $-0.325 * *$ & $0.080 * *$ & $-0.347 * *$ & $-2.311 * *$ & $19.439 * *$ & $-12.135^{* *}$ & -0.647 & $-7.859^{*}$ \\
\hline 5 & EC-41752 x Ex-7959 & $12.903 * *$ & $14.706 * *$ & $0.332 * *$ & $-0.042 * *$ & $0.343^{* *}$ & $1.500^{* *}$ & $-11.526^{* *}$ & $8.132 * *$ & 0.282 & $7.118^{* *}$ \\
\hline 6 & EC-41752 x Neelam & $-4.922 * *$ & $-7.453 * *$ & -0.007 & $-0.037 * *$ & 0.004 & $0.811^{* *}$ & $-7.912 * *$ & $4.003 * *$ & 0.365 & $0.741 * *$ \\
\hline 7 & EC-98994 x Heera & $-9.839 * *$ & -1.077 & $0.637 * *$ & $-0.049 * *$ & $0.615^{* *}$ & $-0.644 * *$ & $-1.879 * *$ & $-2.818 * *$ & $2.427 * *$ & $6.879 * *$ \\
\hline 8 & EC-98994 x Ex-7959 & 0.945 & 0.256 & -0.022 & $-0.060 * *$ & -0.011 & $0.667 * *$ & $8.255^{* *}$ & $4.308^{* *}$ & $-2.426 * *$ & $-0.309 * *$ \\
\hline 9 & EC-98994 x Neelam & $8.894 * *$ & $0.822 * *$ & $-0.615^{* *}$ & $0.109 * *$ & $-0.604 * *$ & $-0.022 * *$ & $-6.376 * *$ & $-1.491 * *$ & -0.001 & $-6.569 * *$ \\
\hline 10 & T-397 x Heera & $-4.430 * *$ & $4.119 *$ & $0.557 * *$ & $0.386^{* *}$ & $0.534 * *$ & $-0.978^{* *}$ & $3.488^{* *}$ & $-7.170 * *$ & $-4.566 * *$ & $1.716^{* *}$ \\
\hline 11 & T-397 x Ex-7959 & 1.353 & $7.017 * *$ & $-0.227 * *$ & $-0.114 * *$ & $-0.216 * *$ & $1.333^{* *}$ & $-12.855^{* *}$ & $4.840^{* *}$ & 0.448 & $3.751 * *$ \\
\hline 12 & T-397 x Neelam & 3.078 & $-11.137 * *$ & $-0.330^{* *}$ & $-0.273 * *$ & $-0.319 * *$ & $-0.356^{* *}$ & $9.367 * *$ & $2.331 * *$ & $4.118^{* *}$ & $-5.467 * *$ \\
\hline 13 & EC-99029 x Heera & 0.784 & $-6.886 * *$ & $0.305^{* *}$ & $0.002 * *$ & $0.283^{* *}$ & $-0.089 * *$ & $2.220 * *$ & $0.681 * *$ & $-2.712 * *$ & $-0.470 * *$ \\
\hline 14 & EC-99029 x Ex-7959 & $3.967 *$ & $6.827 * *$ & $-0.564 * *$ & $-0.133^{* *}$ & $-0.553 * *$ & $-0.444 * *$ & $-6.426 * *$ & $-2.987 * *$ & 0.824 & $2.706^{* *}$ \\
\hline 15 & EC-99029 x Neelam & $-4.751 *$ & 0.058 & $0.259 * *$ & $0.131^{* *}$ & $0.270^{* *}$ & $0.533 * *$ & $4.206^{* *}$ & $2.305^{* *}$ & $1.888^{* *}$ & $-2.236 * *$ \\
\hline 16 & EC-99056 x Heera & $8.695 * *$ & $-7.112 * *$ & $-0.214 * *$ & $-0.072 * *$ & $-0.237 * *$ & $0.189^{*}$ & 0.244 & $1.340 * *$ & -0.233 & $-2.227 * *$ \\
\hline 17 & EC-99056 x Ex-7959 & 0.038 & $13.756^{* *}$ & $0.146^{*}$ & $-0.020 * *$ & 0.157 & $-1.000 * *$ & $1.005 * *$ & $-4.323 * *$ & 1.224 & $5.752 * *$ \\
\hline 18 & EC-99056 x Neelam & $-8.732 * *$ & $-6.643 * *$ & 0.068 & $0.091 * *$ & 0.080 & $0.811^{* *}$ & $-1.250 * *$ & $2.983^{* *}$ & -0.991 & $-3.524 * *$ \\
\hline 19 & EC-104739 x Heera & $15.406 * *$ & 2.091 & $-0.388 * *$ & $0.109 * *$ & $-0.410 * *$ & $0.189^{*}$ & $-10.349 * *$ & $-0.133^{* *}$ & $6.829 * *$ & $3.346^{* *}$ \\
\hline 20 & EC-104739 x Ex-7959 & $-10.361 * *$ & 1.149 & 0.136 & $-0.036^{* *}$ & 0.147 & $-1.000 * *$ & $-15.945^{* *}$ & $-4.130 * *$ & -1.392 & $-0.007 * *$ \\
\hline 21 & EC-104739 x Neelam & $-5.046 * *$ & -3.240 & $0.252 * *$ & $-0.072 * *$ & $0.263 * *$ & $0.811 * *$ & $26.293 * *$ & $4.263^{* *}$ & $-5.437 * *$ & $-3.339 * *$ \\
\hline 22 & CRISTA x Heera & -1.745 & $-12.141 * *$ & -0.114 & $0.017 * *$ & -0.136 & $0.811 * *$ & 0.123 & $0.399 * *$ & $-6.157 * *$ & $4.595 * *$ \\
\hline 23 & CRISTA x Ex-7959 & 3.087 & -1.280 & $-0.472 * *$ & $-0.201 * *$ & $-0.461 * *$ & $-1.000 * *$ & $6.531 * *$ & $-2.408 * *$ & $3.952 * *$ & $-3.999 * *$ \\
\hline 24 & CRISTA x Neelam & -1.341 & $13.241 * *$ & $0.586^{* *}$ & $0.184 * *$ & $0.598^{* *}$ & $0.811^{* *}$ & $-6.653 * *$ & $2.008 * *$ & $2.205^{* *}$ & $-0.596 * *$ \\
\hline 25 & EC-110288 x Heera & $-9.256 * *$ & $7.048 * *$ & -0.067 & $0.026^{* *}$ & -0.090 & $2.133 * *$ & $-0.475^{*}$ & $12.695^{* *}$ & 0.226 & $2.555^{* *}$ \\
\hline 26 & EC-110288 x Ex-7959 & $-10.090^{* *}$ & $-12.367 * *$ & -0.060 & $-0.063 * *$ & -0.049 & $-0.722 * *$ & $1.492 * *$ & $-3.686^{* *}$ & $-2.329 * *$ & $-5.180 * *$ \\
\hline 27 & EC-110288 x Neelam & $19.346^{* *}$ & $5.319 * *$ & 0.128 & $0.037 * *$ & 0.139 & $1.411^{* *}$ & $-1.017 * *$ & $-9.009 * *$ & $2.103 * *$ & $2.634 * *$ \\
\hline 28 & EC-112689 x Heera & $-4.830 * *$ & -0.429 & $-0.146^{*}$ & $-0.074 * *$ & -0.168 & -1.200 & $0.406^{* *}$ & $-6.570 * *$ & $1.891 *$ & $1.774 * *$ \\
\hline 29 & EC-112689 x Ex-7959 & $-9.893 * *$ & 2.119 & $-0.384 * *$ & $-0.049 * *$ & $-0.373 * *$ & 0.111 & $5.494 * *$ & $0.111^{* *}$ & 1.326 & $-1.754 * *$ \\
\hline 30 & EC-112689 x Neelam & $14.723 * *$ & -1.690 & $0.529 * *$ & $0.123 * *$ & $0.540 * *$ & $1.089 * *$ & $-5.899 * *$ & $6.460 * *$ & $-3.217 * *$ & $-0.020 * *$ \\
\hline 31 & EC-115161 x Heera & $6.566^{* *}$ & -3.339 & $0.320 * *$ & $0.005^{* *}$ & $0.298^{* *}$ & -1.200 & $-5.119 * *$ & $-7.438^{* *}$ & 1.075 & $7.933^{* *}$ \\
\hline 32 & EC-115161 x Ex-7959 & $-7.256 * *$ & $16.444 * *$ & $-0.346^{* *}$ & $-0.004 * *$ & $-0.335 * *$ & 0.111 & $5.022 * *$ & $-0.345^{* *}$ & $3.659 * *$ & $4.756^{* *}$ \\
\hline 33 & EC-115161 x Neelam & 0.691 & $-13.105^{* *}$ & 0.026 & $-0.001 * *$ & 0.037 & $1.089^{* *}$ & 0.097 & $7.782 * *$ & $-4.734 * *$ & $-12.689 * *$ \\
\hline 34 & EC-115162 x Heera & $5.779 * *$ & $-14.977 * *$ & $-0.318^{* *}$ & $-0.238^{* *}$ & $-0.340 * *$ & $-0.644 * *$ & $2.445 * *$ & $-0.931 * *$ & 0.939 & $-2.501 * *$ \\
\hline 35 & EC-115162 x Ex-7959 & -2.749 & $-6.394 * *$ & 0.112 & $0.077 * *$ & 0.123 & $0.667 * *$ & $-0.488^{*}$ & $2.935^{* *}$ & $2.644 * *$ & $1.906^{* *}$ \\
\hline 36 & EC-115162 x Neelam & -3.030 & $21.372 * *$ & $0.206^{* *}$ & $0.161 * *$ & $0.217 * *$ & -0.022 & $-1.956 * *$ & $-2.004 * *$ & $-3.582 * *$ & $0.594 * *$ \\
\hline 37 & EC-115178 x Heera & $13.284 * *$ & $3.851^{*}$ & $0.652 * *$ & $0.261 * *$ & $0.630 * *$ & $1.578 * *$ & $-15.305^{* *}$ & $6.640 * *$ & -0.096 & $-0.929 * *$ \\
\hline 38 & EC-115178 x Ex-7959 & -1.333 & -3.116 & $0.199 * *$ & $-0.065^{* *}$ & $0.210^{* *}$ & $-1.278 * *$ & $-7.581 * *$ & $-7.667 * *$ & $-3.544 * *$ & $-3.784 * *$ \\
\hline 39 & EC-115178 x Neelam & $-11.951 * *$ & -0.735 & $-0.851 * *$ & $-0.165 * *$ & $-0.840 * *$ & $-0.300 * *$ & $22.887 * *$ & $1.027 * *$ & $3.640 * *$ & $4.713 * *$ \\
\hline 40 & EC-115186 x Heera & $16.596 * *$ & 0.258 & $-0.835^{* *}$ & $-0.324 * *$ & $-0.858 * *$ & $-0.922 * *$ & $7.940 * *$ & $-0.361 * *$ & 1.495 & $1.569 * *$ \\
\hline 41 & EC-115186 x Ex-7959 & -2.949 & $-7.454 * *$ & $0.670 * *$ & $-0.051 * *$ & $0.681 * *$ & $1.278 * *$ & $16.721 * *$ & $-8.249 * *$ & 0.155 & $1.408 * *$ \\
\hline 42 & EC-115186 x Neelam & $-13.647 * *$ & $7.197 * *$ & $0.166^{*}$ & $0.375 * *$ & 0.177 & $2.200 * *$ & $-24.661 * *$ & $8.609 * *$ & $-1.650 *$ & $-2.978 * *$ \\
\hline 43 & EC-225125 x Heera & 0.339 & $-4.757 * *$ & $0.185^{* *}$ & $-0.060 * *$ & 0.163 & $1.022 * *$ & $-3.668 * *$ & $5.409 * *$ & -0.0184 & $-3.350 * *$ \\
\hline 44 & EC-225125 x Ex-7959 & $3.730 *$ & 2.661 & $-0.614 * *$ & $-0.058 * *$ & $-0.603 * *$ & $-0.167 * *$ & $8.245^{* *}$ & $0.228 * *$ & -1.260 & $3.224 * *$ \\
\hline 45 & EC-225125 x Neelam & $-4.068 *$ & 2.097 & $0.429 * *$ & $0.118 * *$ & $0.440 * *$ & $-0.856^{* *}$ & $-4.576^{* *}$ & $-5.637 * *$ & 1.444 & $0.126 * *$ \\
\hline
\end{tabular}


Int.J.Curr.Microbiol.App.Sci (2019) 8(1): 1118-1148

Continued....

\begin{tabular}{|c|c|c|c|c|c|c|c|c|c|c|c|}
\hline S.No & Crosses & & & $\mathrm{BD}$ & & TD & & $\mathrm{PO}$ & & PR & \\
\hline & & $\mathrm{F}_{1}$ & $\mathrm{~F}_{2}$ & $\mathrm{~F}_{1}$ & $\mathrm{~F}_{2}$ & $\mathrm{~F}_{1}$ & $\mathrm{~F}_{2}$ & $\mathrm{~F}_{1}$ & $\mathrm{~F}_{2}$ & $\mathrm{~F}_{1}$ & $\mathrm{~F}_{2}$ \\
\hline 46 & EC-1041492 x Heera & $-4.060 *$ & -4.227 & $-0.174 * *$ & $0.048^{* *}$ & $-0.196^{*}$ & $-0.644 * *$ & $4.740 * *$ & $-7.682 * *$ & $-2.192 * *$ & $-2.745^{* *}$ \\
\hline 47 & EC-1041492 x Ex-7959 & -1.825 & -3.584 & 0.130 & $-0.113 * *$ & 0.141 & $0.667 * *$ & $3.817 * *$ & $12.596^{* *}$ & $6.064 * *$ & $-1.969 * *$ \\
\hline 48 & EC-1041492 x Neelam & $5.886 * *$ & $7.812 * *$ & 0.044 & $0.065^{* *}$ & 0.055 & -0.022 & $-8.557 * *$ & $-4.914 * *$ & $-3.873 * *$ & $4.713^{* *}$ \\
\hline 49 & Mukta x Heera & $-4.417^{*}$ & -0.491 & -0.011 & $0.118 * *$ & -0.033 & -0.089 & $0.507 *$ & $-0.450 * *$ & -0.096 & $-0.187 * *$ \\
\hline 50 & Mukta x Ex-7959 & $-7.022 * *$ & -1.143 & $-0.240 * *$ & $-0.032 * *$ & $-0.228 * *$ & $1.222 * *$ & $5.476^{* *}$ & $8.063 * *$ & $-5.755^{* *} *$ & $3.487 * *$ \\
\hline 51 & Mukta x Neelam & $11.439 * *$ & 1.903 & $0.250^{* *}$ & $-0.086 * *$ & $0.261^{* *}$ & $-1.133 * *$ & $-5.983 * *$ & $-7.613 * *$ & $5.851^{* *}$ & $-3.300^{* *}$ \\
\hline 52 & ES-1463 x Heera & $-7.418 * *$ & $-7.602 * *$ & $-0.827 * *$ & $0.346^{* *}$ & $-0.849 * *$ & -0.089 & $9.472 * *$ & $-2.977 * *$ & -0.623 & $-5.507 * *$ \\
\hline 53 & ES-1463 x Ex-7959 & $-12.207 * *$ & $12.486^{* *}$ & 0.057 & $-0.167 * *$ & 0.069 & $-0.444 * *$ & $1.207 * *$ & $-2.204 * *$ & $2.634 * *$ & $0.686^{* *}$ \\
\hline 54 & ES-1463 x Neelam & $19.625 * *$ & $-4.883 *$ & $0.770 * *$ & $-0.179 * *$ & $0.781 * *$ & $0.533^{* *}$ & $-10.679 * *$ & $5.181 * *$ & $-2.011 * *$ & $4.820 * *$ \\
\hline 55 & ES-1474 x Heera & $-6.534 * *$ & $14.873^{* *}$ & $0.372 * *$ & $0.076^{* *}$ & $0.350^{* *}$ & $1.022 * *$ & $0.800 * *$ & $4.291 * *$ & $1.388^{*}$ & $3.004^{* *}$ \\
\hline 56 & ES-1474 x Ex-7959 & 0.764 & $-5.469^{*}$ & $-0.143^{*}$ & $0.080^{* *}$ & -0.132 & $-0.167 * *$ & $9.235^{* *}$ & $-1.266^{* *}$ & $-1.255^{*}$ & $-2.222 * *$ \\
\hline 57 & ES-1474 x Neelam & $5.769 * *$ & $-9.403 * *$ & $-0.228 * *$ & $-0.156 * *$ & $-0.217^{*}$ & $-0.856^{* *}$ & $-10.036^{* *}$ & $-3.025 * *$ & -0.132 & $-0.782 * *$ \\
\hline 58 & ES-1496 x Heera & $7.510^{* *}$ & $5.484 *$ & 0.019 & $-0.421 * *$ & -0.003 & $-0.644 * *$ & $-1.598 * *$ & $0.899 * *$ & $-2.385 * *$ & $-3.662 * *$ \\
\hline 59 & ES-1496 x Ex-7959 & $6.524 * *$ & -0.138 & $0.360 * *$ & $0.366^{* *}$ & $0.371 * *$ & $0.667 * *$ & $-3.362 * *$ & $0.048 * *$ & $1.238^{*}$ & $-1.715^{* *}$ \\
\hline 60 & ES-1496 x Neelam & $-14.034 * *$ & $-5.347 * *$ & $-0.379 * *$ & $0.055^{* *}$ & $-0.368 * *$ & -0.022 & $4.959 * *$ & $-0.947 * *$ & $1.146^{*}$ & $5.377 * *$ \\
\hline 61 & ES-1531 x Heera & $3.728^{*}$ & $6.498^{* *}$ & $-0.944 * *$ & $0.039 * *$ & $-0.966 * *$ & $1.022 * *$ & $1.502 * *$ & $4.448 * *$ & $1.207 *$ & $-5.038 * *$ \\
\hline 62 & ES-1531 x Ex-7959 & -0.812 & -3.355 & $0.544 * *$ & $0.099 * *$ & $0.555^{* *}$ & $-0.167 * *$ & $-14.273 * *$ & $-1.351 * *$ & $-3.968 * *$ & $-5.785^{* *}$ \\
\hline 63 & ES-1531 x Neelam & -2.917 & -3.144 & $0.400 * *$ & $-0.139 * *$ & $0.411^{* *}$ & $-0.856 * *$ & $12.771 * *$ & $-3.097 * *$ & $2.762^{* *}$ & $10.823 * *$ \\
\hline 64 & ES-14600 x Heera & 2.504 & -1.417 & $0.335^{* *}$ & $0.042 * *$ & $0.313 * *$ & -0.089 & $1.169^{* *}$ & $0.279 * *$ & $-2.126 * *$ & $-4.628 * *$ \\
\hline 65 & ES-14600 x Ex-7959 & $-8.056^{* *}$ & $-9.999 * *$ & $0.574 * *$ & $-0.159 * *$ & $0.585^{* *}$ & $-0.444 * *$ & $5.639 * *$ & $-2.730 * *$ & $-1.841^{* *}$ & $0.920 * *$ \\
\hline 66 & ES-14600 x Neelam & $5.552 * *$ & $11.417 * *$ & $-0.909 * *$ & $0.117 * *$ & $-0.898 * *$ & $0.533^{* *}$ & $-6.808 * *$ & $2.450 * *$ & $3.967 * *$ & $3.708^{* *}$ \\
\hline 67 & Ex-3 x Heera & $-9.310 * *$ & $-6.364 *$ & -0.034 & $0.078^{* *}$ & -0.056 & $1.578^{* *}$ & $-1.013^{* *}$ & $8.593 * *$ & $-1.784 * *$ & $-0.509 * *$ \\
\hline 68 & Ex-3 x Ex-7959 & $22.790 * *$ & $-7.666 * *$ & -0.089 & $0.045^{* *}$ & -0.078 & $-1.278 * *$ & $1.995^{* *}$ & $-9.415 * *$ & -0.021 & $-1.403 * *$ \\
\hline 69 & Ex-3 x Neelam & $-13.480 * *$ & $14.030 * *$ & 0.123 & $-0.123 * *$ & 0.134 & $-0.300 * *$ & $-0.981 * *$ & $0.822 * *$ & $1.805^{* *}$ & $1.912 * *$ \\
\hline 70 & Ex-5-36E x Heera & -1.392 & 4.338 & $0.275^{* *}$ & $0.080^{* *}$ & $0.253^{* *}$ & $1.578^{* *}$ & $-13.006^{* *}$ & $8.030 * *$ & 0.016 & $-0.549 * *$ \\
\hline 71 & Ex-5-36E x Ex-7959 & $-6.258^{* *}$ & $9.891 * *$ & 0.026 & $0.083^{* *}$ & 0.037 & $0.389 * *$ & $17.096^{* *}$ & $2.724 * *$ & 0.829 & $-0.710 * *$ \\
\hline 72 & Ex-5-36E x Neelam & $7.650 * *$ & $-14.228 * *$ & $-0.301 * *$ & $0.163^{* *}$ & $-0.290 * *$ & $-1.967 * *$ & $-4.090 * *$ & $-10.574 * *$ & -0.845 & $1.258^{* *}$ \\
\hline 73 & Ex-28-3 x Heera & $-6.006^{* *}$ & $4.956^{* *}$ & $0.624 * *$ & $-0.157 * *$ & $0.602 * *$ & -0.089 & $-17.097 * *$ & $2.483 * *$ & $2.456^{* *}$ & $-2.754 * *$ \\
\hline 74 & Ex-28-3 x Ex-7959 & $11.973 * *$ & $-10.491 * *$ & -0.043 & $0.053^{* *}$ & -0.032 & $1.222 * *$ & $17.930 * *$ & $7.386^{* *}$ & $3.342 * *$ & $1.887 * *$ \\
\hline 75 & Ex-28-3 x Neelam & $-5.967 * *$ & $5.535^{* *}$ & $-0.581 * *$ & $0.104 * *$ & $0.570^{* *}$ & $-1.133 * *$ & $-0.833 * *$ & $-9.870 * *$ & $-5.798 * *$ & $0.868^{* *}$ \\
\hline 76 & Ex-313-23 x Heera & -0.810 & 0.914 & 0.081 & $-0.406^{* *}$ & 0.059 & $-1.200 * *$ & $-2.277 * *$ & $-2.630 * *$ & $-3.686^{* *}$ & $2.340 * *$ \\
\hline 77 & Ex-313-23 x Ex-7959 & 4.112 & $-5.288 * *$ & $0.647 * *$ & $0.222^{* *}$ & $0.658 * *$ & 0.111 & $-6.909 * *$ & $-3.177 * *$ & 0.136 & $-3.355^{* *}$ \\
\hline 78 & Ex-313-23 x Neelam & -3.302 & 4.373 & $-0.728 * *$ & $0.183^{* *}$ & $-0.717 * *$ & $1.089^{* *}$ & $9.186^{* *}$ & $5.807 * *$ & $3.733 * *$ & $1.014 * *$ \\
\hline 79 & Ex-339-6 x Heera & $5.586^{* *}$ & 0.553 & $0.228 * *$ & $0.173^{* *}$ & $0.205^{* *}$ & $0.578^{* *}$ & $-4.136 * *$ & $-0.904 * *$ & $2.044^{* *}$ & $2.194 * *$ \\
\hline 80 & Ex-339-6 x Ex-7959 & $3.312 *$ & $7.376^{* *}$ & $-0.271 * *$ & $-0.046 * *$ & $-0.260 * *$ & $-0.778 * *$ & $4.159 * *$ & $-4.112 * *$ & $1.275^{*}$ & $-0.339 * *$ \\
\hline 81 & Ex-339-6 x Neelam & $-8.898 * *$ & $-7.928 * *$ & 0.044 & $-0.127 * *$ & $0.055^{* *}$ & $0.200 * *$ & -0.022 & $5.017 * *$ & $-3.319 * *$ & $1.855^{* *}$ \\
\hline 82 & Ezox Natural x Heera & 0.529 & $14.753 * *$ & $-0.295 * *$ & $-0.175 * *$ & $-0.317 * *$ & $-0.644 * *$ & $9.722 * *$ & $-1.558 * *$ & $7.102^{* *}$ & $1.696^{* *}$ \\
\hline 83 & Ezox Natural x Ex-7959 & -1.745 & -4.674 & $0.325^{* *}$ & $-0.023 * *$ & $0.336^{* *}$ & $0.667 * *$ & $-14.705^{* *}$ & $3.932 * *$ & $-2.656^{* *}$ & $-0.749 * *$ \\
\hline 84 & Ezox Natural x Neelam & 1.215 & $-10.078 * *$ & -0.030 & $0.198 * *$ & -0.019 & -0.022 & $8.983 * *$ & $-2.374 * *$ & $-4.446 * *$ & $-0.948 * *$ \\
\hline 85 & FR-3 $\times$ Heera & $-9.856^{* *}$ & 0.983 & $0.364 * *$ & $0.118^{* *}$ & $0.341 * *$ & -0.644 & $3.475 * *$ & $-4.269 * *$ & $-2.316 * *$ & $-3.467 * *$ \\
\hline 86 & FR-3 x Ex-7959 & $4.748 *$ & -2.484 & $-0.448 * *$ & $0.070^{* *}$ & $-0.437 * *$ & $0.667 * *$ & $-10.530^{* *}$ & $3.149 * *$ & $-1.358^{*}$ & $0.325^{* *}$ \\
\hline 87 & FR-3 x Neelam & $5.107 * *$ & 1.502 & 0.084 & $-0.188 * *$ & 0.095 & 0.022 & $7.055^{* *}$ & $1.121 * *$ & $3.674 * *$ & $3.142 * *$ \\
\hline 88 & FRW-9 $x$ Heera & $-3.767 *$ & -2.401 & $-0.155^{*}$ & $0.017 * *$ & $-0.177 * *$ & $1.022^{* *}$ & $8.283^{* * *}$ & $4.674 * *$ & 0.802 & $1.530^{* *}$ \\
\hline 89 & FRW-9 x Ex-7959 & -1.707 & $-7.203 * *$ & -0.006 & $0.060^{* *}$ & 0.005 & -0.167 & $-15.482 * *$ & $-0.973 * *$ & -0.875 & $-5.746^{* *}$ \\
\hline \multirow[t]{2}{*}{90} & FRW-9 x Neelam & $5.474 * *$ & $9.603^{* *}$ & $0.161^{*}$ & $0.008^{* *}$ & $0.172 * *$ & $-0.856 * *$ & $7.198^{* *}$ & $-3.702 * *$ & 0.073 & $4.215^{* *}$ \\
\hline & Sij-skl & 1.846 & 2.463 & 0.069 & 0.0006 & 0.086 & 0.086 & 0.238 & 0.0004 & 0.801 & 0.0001 \\
\hline
\end{tabular}

Continued.... 


\begin{tabular}{|c|c|c|c|c|c|c|c|c|c|c|c|}
\hline S.No & Crosses & FAA & & CAR & & PHE & & RF & & SG & \\
\hline & & $F_{1}$ & $\mathrm{~F}_{2}$ & $\mathrm{~F}_{1}$ & $\mathrm{~F}_{2}$ & $\mathrm{~F}_{1}$ & $\mathrm{~F}_{2}$ & $F_{1}$ & $\mathrm{~F}_{2}$ & $\mathrm{~F}_{1}$ & $\mathrm{~F}_{2}$ \\
\hline 1 & EC-41750 x Heera & 0.002 & $0.004 * *$ & $-1.021 * *$ & $6.980 * *$ & $0.058^{* *}$ & $-1.608 * *$ & -0.005 & $-0.026 * *$ & -0.006 & 0.029 \\
\hline 2 & EC-41750 x Ex-7959 & -0.002 & 0.001 & $-5.605 * *$ & $2.161 * *$ & $0.168^{* *}$ & $3.261 * *$ & 0.001 & 0.006 & 0.006 & -0.014 \\
\hline 3 & EC-41750 x Neelam & -0.001 & $-0.004 * *$ & $6.626^{* *}$ & $-9.140 * *$ & $-0.226^{* *}$ & $-1.653 * *$ & 0.005 & $0.020^{* *}$ & 0.001 & -0.015 \\
\hline 4 & EC-41752 x Heera & $0.004 * *$ & $-0.008 * *$ & $-4.398 * *$ & $0.789 * *$ & $-0.869 * *$ & $-0.753^{* *}$ & 0.005 & 0.004 & $-0.022 * *$ & -0.020 \\
\hline 5 & EC-41752 x Ex-7959 & $-0.003^{*}$ & $0.007 * *$ & $1.535^{* *}$ & $4.227 * *$ & $1.226^{* *}$ & $0.899 * *$ & -0.006 & $-0.11 * *$ & 0.003 & -0.026 \\
\hline 6 & EC-41752 x Neelam & 0.001 & 0.001 & $2.863 * *$ & $-5.017 * *$ & $-0.357 * *$ & $-0.146 * *$ & 0.001 & 0.007 & $0.019 * *$ & 0.047 \\
\hline 7 & EC-98994 x Heera & 0.001 & $0.007 * *$ & $-4.265^{* *}$ & $4.980 * *$ & $-0.964 * *$ & $0.652 * *$ & $-0.025 * *$ & $0.015^{* *}$ & 0.003 & 0.006 \\
\hline 8 & EC-98994 x Ex-7959 & $-0.003 *$ & 0.001 & $5.243 * *$ & $-3.353 * *$ & $2.091 * *$ & $0.137 * *$ & $0.053 * *$ & -0.001 & -0.007 & 0.005 \\
\hline 9 & EC-98994 x Neelam & $0.003 *$ & $-0.007 * *$ & $-0.978 * *$ & $-1.627 * *$ & $-1.127 * *$ & $-0.790 * *$ & $-0.028 * *$ & $-0.014 * *$ & 0.004 & -0.10 \\
\hline 10 & T-397 x Heera & $0.005^{* *}$ & $0.002 * *$ & $-1.109 * *$ & $-9.420 * *$ & $0.789 * *$ & $1.279 * *$ & 0.001 & -0.007 & 0.001 & -0.027 \\
\hline 11 & T-397 x Ex-7959 & -0.001 & $0.004 * *$ & $-1.146^{* *}$ & $-5.096^{* *}$ & $0.902 * *$ & $1.049 * *$ & -0.002 & 0.005 & $-0.015 * *$ & -0.007 \\
\hline 12 & T-397 x Neelam & $-0.003 *$ & $-0.005^{* *}$ & $1.255^{* *}$ & $14.515^{* *}$ & $-1.692 * *$ & $-2.328 * *$ & 0.003 & 0.002 & $0.014 * *$ & 0.033 \\
\hline 13 & EC-99029 x Heera & $0.003^{*}$ & 0.001 & $-1.403 * *$ & $-2.639 * *$ & $0.572 * *$ & $0.216^{* *}$ & 0.002 & -0.004 & $0.028 * *$ & -0.009 \\
\hline 14 & EC-99029 x Ex-7959 & $-0.005 * *$ & $0.003 * *$ & $6.812 * *$ & $-1.287 * *$ & $1.437 * *$ & $0.840 * *$ & -0.003 & -0.005 & $-0.030 * *$ & 0.001 \\
\hline 15 & EC-99029 x Neelam & 0.002 & $-0.002 * *$ & $-5.409 * *$ & $3.926 * *$ & $-2.009 * *$ & $-1.056^{* *}$ & 0.002 & 0.008 & 0.001 & 0.009 \\
\hline 16 & EC-99056 x Heera & $-0.005 * *$ & $-0.002 * *$ & $0.054 * *$ & $-11.543 * *$ & $-0.246^{* * *}$ & $-0.226 * *$ & 0.005 & 0.001 & 0.005 & 0.011 \\
\hline 17 & EC-99056 x Ex-7959 & $0.009 * *$ & $0.006^{* *}$ & $-7.042 * *$ & $10.894 * *$ & $-0.158 * *$ & $1.876^{* *}$ & -0.008 & -0.006 & 0.001 & -0.045 \\
\hline 18 & EC-99056 x Neelam & $-0.004 * *$ & $-0.004 * *$ & $6.988 * *$ & $0.649 * *$ & $0.404^{* *}$ & $-1.650 * *$ & 0.003 & 0.006 & -0.007 & 0.034 \\
\hline 19 & EC-104739 x Heera & -0.001 & $0.004 * *$ & $1.596^{* *}$ & $-3.934 * *$ & $-0.585^{* *}$ & $-0.092 * *$ & 0.001 & 0.004 & 0.011 & 0.019 \\
\hline 20 & EC-104739 x Ex-7959 & $-0.005 * *$ & 0.001 & $-9.028 * *$ & $7.732 * *$ & $-0.574 * *$ & $-0.817 * *$ & -0.011 & -0.004 & $0.032 * *$ & -0.053 \\
\hline 21 & EC-104739 x Neelam & $0.006^{* *}$ & $-0.004 * *$ & $7.432 * *$ & $-3.798 * *$ & $1.159 * *$ & $0.909 * *$ & 0.011 & 0.001 & $-0.043 * *$ & 0.034 \\
\hline 22 & CRISTA $x$ Heera & $-0.003 *$ & $0.005^{* *}$ & $-3.107 * *$ & $-2.810 * *$ & $0.647 * *$ & $-0.736 * *$ & 0.002 & $0.035^{* *}$ & $-0.027 * *$ & -0.004 \\
\hline 23 & CRISTA x Ex-7959 & -0.001 & $-0.004 * *$ & $-8.232 * *$ & $-0.230 * *$ & $0.282 * *$ & $-0.106 * *$ & 0.004 & $-0.013 * *$ & $0.015^{*}$ & -0.033 \\
\hline 24 & CRISTA x Neelam & $0.003 *$ & -0.001 & $11.339 * *$ & $3.040 * *$ & $-0.929 * *$ & $0.842 * *$ & -0.005 & $-0.022 * *$ & 0.012 & 0.037 \\
\hline 25 & EC-110288 x Heera & $-0.003 * *$ & $0.003 * *$ & $-1.060 * *$ & $-4.534 * *$ & $2.449 * *$ & $2.197 * *$ & 0.004 & $-0.012 * *$ & 0.004 & -0.033 \\
\hline 26 & EC-110288 x Ex-7959 & $0.004 * *$ & $-0.005 * *$ & $-1.185^{* *}$ & $1.447 * *$ & $-2.532 * *$ & $-2.079 * *$ & 0.002 & 0.008 & 0.004 & 0.001 \\
\hline 27 & EC-110288 x Neelam & -0.002 & $0.003 * *$ & $2.245^{* *}$ & $30.87 * *$ & $0.083^{* *}$ & $-0.118 * *$ & -0.006 & 0.004 & -0.008 & 0.032 \\
\hline 28 & EC-112689 x Heera & 0.001 & $0.002 * *$ & $-6.430 * *$ & $2.028 * *$ & $2.354 * *$ & $0.310 * *$ & 0.005 & $0.030^{* *}$ & 0.005 & 0.056 \\
\hline 29 & EC-112689 x Ex-7959 & $-0.003 * *$ & $-0.002 * *$ & $3.155^{* *}$ & $-0.277 * *$ & $-2.184 * *$ & $-0.698 * *$ & 0.001 & $0.029 * *$ & -0.009 & -0.218 ** \\
\hline 30 & EC-112689 x Neelam & 0.002 & 0.001 & $3.275^{* *}$ & $-1.750 * *$ & $-0.169 * *$ & $0.387 * *$ & -0.005 & $-0.060 * *$ & 0.004 & $0.162 * *$ \\
\hline 31 & EC-115161 x Heera & 0.001 & $0.008 * *$ & $1.028 * *$ & $5.542 * *$ & $-0.240 * *$ & $-0.243 * *$ & -0.002 & 0.005 & 0.005 & 0.049 \\
\hline 32 & EC-115161 x Ex-7959 & 0.001 & $0.005^{* *}$ & $4.788 * *$ & $9.065 * *$ & $0.710 * *$ & $0.518 * *$ & -0.001 & $-0.019 * *$ & -0.002 & -0.040 \\
\hline 33 & EC-115161 x Neelam & -0.002 & $-0.013 * *$ & $-5.813 * *$ & $-14.607 * *$ & $-0.470 * *$ & $-0.275^{* *}$ & 0.003 & $0.014 * *$ & -0.003 & -0.009 \\
\hline 34 & EC-115162 x Heera & 0.002 & $-0.002 * *$ & $1.249 * *$ & $-0.610 * *$ & $-1.873 * *$ & $0.540 * *$ & -0.006 & 0.001 & 0.001 & -0.004 \\
\hline 35 & EC-115162 x Ex-7959 & $0.003 *$ & $0.002 * *$ & $4.511 * *$ & $3.999 * *$ & $-0.778^{* *}$ & $0.110^{* *}$ & 0.003 & $0.016^{* *}$ & -0.003 & 0.063 \\
\hline 36 & EC-115162 x Neelam & $-0.005 * *$ & 0.001 & $-3.262 * *$ & $-3.388 * *$ & $2.651 * *$ & $-0.650 * *$ & 0.004 & $-0.017 * *$ & 0.002 & -0.058 \\
\hline 37 & EC-115178 x Heera & $-0.003 *$ & -0.001 & $4.593^{* *}$ & $-2.315^{* *}$ & $0.657 * *$ & $0.654 * *$ & 0.001 & $-0.030 * *$ & 0.005 & 0.015 \\
\hline 38 & EC-115178 x Ex-7959 & 0.002 & $-0.004 * *$ & $-2.356^{* *}$ & $-4.191 * *$ & $-1.403 * *$ & $-0.693 * *$ & -0.005 & 0.004 & -0.001 & 0.015 \\
\hline 39 & EC-115178 x Neelam & 0.001 & $0.005^{* *}$ & $-2.237 * *$ & $6.506 * *$ & $0.747 * *$ & $0.039 * *$ & 0.006 & $0.025^{* *}$ & -0.003 & -0.030 \\
\hline 40 & EC-115186 x Heera & $0.005 * *$ & $0.002 * *$ & $7.108 * *$ & $1.209 * *$ & $1.342 * *$ & $1.091 * *$ & 0.004 & -0.002 & 0.003 & 0.001 \\
\hline 41 & EC-115186 x Ex-7959 & -0.002 & 0.001 & $-6.284 * *$ & $-0.725 * *$ & $-1.330 * *$ & $-2.090 * *$ & -0.009 & 0.001 & -0.008 & 0.011 \\
\hline 42 & EC-115186 x Neelam & $-0.003^{*}$ & $-0.003 * *$ & $-0.824 * *$ & $-0.484 * *$ & $-0.011 * *$ & $0.999 * *$ & 0.005 & 0.002 & 0.004 & -0.012 \\
\hline 43 & EC-225125 x Heera & $-0.004 * *$ & $-0.003 * *$ & $-4.838 * *$ & $1.323 * *$ & $-1.387 * *$ & $0.762 * *$ & 0.006 & $0.015^{* *}$ & $-0.024 * *$ & 0.007 \\
\hline 44 & EC-225125 x Ex-7959 & $0.004 * *$ & $0.003 * *$ & $8.874 * *$ & $-1.410 * *$ & $1.803^{* *}$ & $-0.248 * *$ & 0.001 & 0.005 & $0.019 * *$ & 0.012 \\
\hline 45 & EC-225125 x Neelam & 0.001 & 0.001 & $-4.035^{* *}$ & $0.088^{* *}$ & $-0.416^{* *}$ & $-0.514 * *$ & -0.007 & $-0.020 * *$ & 0.005 & -0.020 \\
\hline
\end{tabular}

Continued.... 


\begin{tabular}{|c|c|c|c|c|c|c|c|c|c|c|c|}
\hline \multirow[t]{2}{*}{ S.No } & \multirow[t]{2}{*}{ Crosses } & \multicolumn{2}{|c|}{ FAA } & \multicolumn{2}{|l|}{ CAR } & \multicolumn{2}{|l|}{ PHE } & \multicolumn{2}{|l|}{$\mathrm{RF}$} & \multicolumn{2}{|l|}{ SG } \\
\hline & & $\mathrm{F}_{1}$ & $\mathrm{~F}_{2}$ & $\mathrm{~F}_{1}$ & $\mathrm{~F}_{2}$ & $\mathrm{~F}_{1}$ & $\mathrm{~F}_{2}$ & $\mathrm{~F}_{1}$ & $\mathrm{~F}_{2}$ & $\mathrm{~F}_{1}$ & $\mathrm{~F}_{2}$ \\
\hline 46 & EC-1041492 x Heera & 0.001 & $-0.003 * *$ & $0.901 * *$ & $0.190 * *$ & $-0.781 * *$ & $-0.926 * *$ & 0.001 & 0.005 & $0.028 * *$ & 0.019 \\
\hline 47 & EC-1041492 x Ex-7959 & 0.001 & $-0.002 * *$ & $-3.967 * *$ & $0.428 * *$ & $-1.144 * *$ & $0.053 * *$ & -0.001 & $-0.014 * *$ & $-0.023^{* *}$ & 0.023 \\
\hline 48 & EC-1041492 x Neelam & $-0.003^{* *}$ & $0.005^{* *}$ & $3.067 * *$ & $-0.617 * *$ & $1.926 * *$ & $0.873 * *$ & 0.001 & $0.009 *$ & -0.004 & -0.042 \\
\hline 49 & Mukta x Heera & 0.001 & 0.001 & $-10.364 * *$ & $2.666^{* *}$ & $-0.382 * *$ & $0.164 * *$ & 0.001 & -0.001 & -0.006 & 0.020 \\
\hline 50 & Mukta x Ex-7959 & 0.001 & $0.004 * *$ & $5.081^{* *}$ & $-3.524 * *$ & $1.356^{* *}$ & $1.364 * *$ & -0.002 & -0.007 & 0.012 & -0.009 \\
\hline 51 & Mukta x Neelam & 0.001 & $-0.003 * *$ & $5.284 * *$ & $0.859 * *$ & $-0.974 * *$ & $-1.528 * *$ & 0.002 & 0.008 & -0.006 & -0.011 \\
\hline 52 & ES-1463 x Heera & $-0.002 *$ & $-0.005 * *$ & $-4.379 * *$ & $0.875^{* *}$ & $-0.126^{* *}$ & $-0.229 * *$ & 0.002 & $0.012 * *$ & $-0.013^{*}$ & 0.024 \\
\hline 53 & ES-1463 x Ex-7959 & $0.005^{* *}$ & 0.001 & $-0.685^{* *}$ & $-9.458 * *$ & $-0.558 * *$ & $-0.812^{* *}$ & -0.003 & -0.003 & $0.016^{*}$ & -0.041 \\
\hline 54 & ES-1463 x Neelam & $-0.003^{* *}$ & $0.005 * *$ & $5.064 * *$ & $8.582 * *$ & $0.684 * *$ & $1.041 * *$ & 0.001 & $-0.009 *$ & -0.003 & 0.018 \\
\hline 55 & ES-1474 x Heera & $-0.007 * *$ & $0.003 * *$ & $3.290 * *$ & $-1.182 * *$ & $0.222 * *$ & $-0.596^{* *}$ & 0.002 & $-0.036^{* *}$ & 0.008 & -0.033 \\
\hline 56 & ES-1474 x Ex-7959 & 0.001 & $-0.002 * *$ & $0.396^{* *}$ & $2.113^{* *}$ & $1.345^{* *}$ & $0.339 * *$ & -0.002 & $0.016^{* *}$ & $-0.010^{*}$ & 0.020 \\
\hline 57 & ES-1474 x Neelam & $0.006^{* *}$ & -0.001 & $-3.686 * *$ & $-0.931 * *$ & $-1.567 * *$ & $0.256^{* *}$ & -0.001 & $0.020^{* *}$ & 0.002 & 0.013 \\
\hline 58 & ES-1496 x Heera & $-0.003 * *$ & $-0.004 * *$ & $2.827 * *$ & $-2.220 * *$ & $-0.838^{* *}$ & $1.112 * *$ & 0.001 & 0.001 & $0.013^{*}$ & -0.062 \\
\hline 59 & ES-1496 x Ex-7959 & $0.003^{* *}$ & $-0.002 * *$ & $-7.253 * *$ & $18.446^{* *}$ & $1.045^{* *}$ & $-0.351^{* *}$ & -0.001 & -0.001 & 0.001 & 0.046 \\
\hline 60 & ES-1496 x Neelam & 0.001 & $0.005^{* *}$ & $4.426 * *$ & $-16.226^{* *}$ & $-0.207 * *$ & $-0.761 * *$ & 0.001 & 0.001 & $-0.014^{*}$ & 0.016 \\
\hline 61 & ES-1531 x Heera & 0.001 & $-0.005^{* *}$ & $14.536^{* *}$ & $6.475^{* *}$ & $-1.772 * *$ & $-1.024 * *$ & 0.001 & $-0.020 * *$ & 0.001 & -0.045 \\
\hline 62 & ES-1531 x Ex-7959 & 0.001 & $-0.006^{* *}$ & $-4.609 * *$ & $-16.257 * *$ & $0.323 * *$ & $-0.558 * *$ & -0.002 & 0.008 & 0.010 & 0.048 \\
\hline 63 & ES-1531 x Neelam & $-0.002 *$ & $0.011 * *$ & $-9.927 * *$ & $9.782 * *$ & $1.449 * *$ & $1.582 * *$ & 0.001 & $0.012 * *$ & -0.011 & -0.003 \\
\hline 64 & ES-14600 x Heera & 0.001 & $-0.005 * *$ & $-1.827 * *$ & $-8.277 * *$ & $0.400^{* *}$ & $0.157 * *$ & 0.001 & -0.006 & $0.031 * *$ & 0.028 \\
\hline 65 & ES-14600 x Ex-7959 & 0.001 & 0.001 & $1.012 * *$ & $11.132 * *$ & $-1.655^{* *}$ & $-1.230 * *$ & 0.001 & $0.009 *$ & $-0.013^{*}$ & 0.024 \\
\hline 66 & ES-14600 x Neelam & $-0.002^{*}$ & $0.004 * *$ & $0.815^{* *}$ & $-2.855^{* *}$ & $1.255^{* *}$ & $1.073 * *$ & 0.001 & -0.003 & $-0.018 * *$ & -0.051 \\
\hline 67 & Ex-3 x Heera & $-0.006^{* *}$ & 0.001 & $0.396^{* *}$ & $0.437 * *$ & $-1.074 * *$ & $-0.244 * *$ & 0.001 & $0.019 * *$ & 0.008 & -0.011 \\
\hline 68 & Ex-3 x Ex-7959 & 0.001 & -0.001 & $0.369 * *$ & $-0.982 * *$ & $-0.259 * *$ & $-0.835^{* *}$ & -0.001 & $-0.010 * *$ & $-0.018^{* *}$ & 0.005 \\
\hline 69 & Ex-3 x Neelam & $0.005^{* *}$ & $0.002 * *$ & $-0.765^{* *}$ & $0.545^{* *}$ & $1.333^{* *}$ & $1.078 * *$ & 0.001 & $-0.009 *$ & 0.010 & 0.006 \\
\hline 70 & Ex-5-36E x Heera & $0.003 * *$ & -0.001 & $-11.402 * *$ & $1.589 * *$ & $0.022 * *$ & $0.017 * *$ & 0.001 & $-0.011 * *$ & $-0.031 * *$ & -0.034 \\
\hline 71 & Ex-5-36E x Ex-7959 & $-0.002^{*}$ & -0.001 & $1.385^{* *}$ & $-2.001 * *$ & $-0.051 * *$ & $-0.203^{* *}$ & -0.001 & 0.005 & $0.023 * *$ & -0.030 \\
\hline 72 & Ex-5-36E x Neelam & -0.001 & 0.001 & $10.017 * *$ & $0.411 * *$ & $0.029 * *$ & $0.185^{* *}$ & 0.001 & 0.006 & 0.008 & 0.063 \\
\hline 73 & Ex-28-3 x Heera & 0.001 & $-0.003 * *$ & $-3.315^{* *}$ & $-2.687 * *$ & $-0.705 * *$ & $-0.042^{* *}$ & 0.001 & 0.001 & 0.002 & -0.021 \\
\hline 74 & Ex-28-3 x Ex-7959 & 0.001 & $0.002 * *$ & $2.911^{* *}$ & $-1.506 * *$ & $0.429 * *$ & $-0.909 * *$ & -0.001 & $0.016^{* *}$ & -0.001 & 0.031 \\
\hline 75 & Ex-28-3 x Neelam & 0.001 & 0.001 & $0.223^{* *}$ & $4.192 * *$ & $0.276^{* *}$ & $0.951^{* *}$ & 0.001 & $-0.016^{* *}$ & -0.001 & -0.010 \\
\hline 76 & Ex-313-23 x Heera & 0.001 & $0.003^{* *}$ & $-2.476 * *$ & $-7.696^{* *}$ & $2.338^{* * *}$ & $0.231 * *$ & -0.001 & 0.001 & -0.012 & -0.005 \\
\hline 77 & Ex-313-23 x Ex-7959 & $-0.005^{* *} *$ & $-0.003 * *$ & $10.091^{* *}$ & $-5.258 * *$ & $-2.237 * *$ & $-1.178^{* *}$ & -0.001 & -0.007 & 0.009 & -0.002 \\
\hline 78 & Ex-313-23 x Neelam & $0.005^{* *}$ & 0.001 & $-7.614 * *$ & $12.954 * *$ & $-0.101 * *$ & $0.947 * *$ & 0.002 & 0.008 & 0.003 & 0.007 \\
\hline 79 & Ex-339-6 x Heera & -0.001 & $0.002 * *$ & $9.338 * *$ & $-5.934 * *$ & $1.108 * *$ & $-0.366^{* *}$ & 0.001 & -0.001 & -0.005 & 0.094 \\
\hline 80 & Ex-339-6 x Ex-7959 & 0.001 & 0.001 & $-3.587 * *$ & $0.732 * *$ & $-0.478 * *$ & $1.023^{* *}$ & -0.001 & 0.001 & $-0.024 * *$ & 0.098 \\
\hline 81 & Ex-339-6 x Neelam & 0.001 & $-0.002 * *$ & $-5.751 * *$ & $5.202 * *$ & $-0.631 * *$ & $-0.658 * *$ & 0.001 & 0.001 & $0.029 * *$ & $-0.191 * *$ \\
\hline 82 & Ezox Natural x Heera & 0.001 & $0.002 * *$ & $4.688^{* *}$ & $-1.182 * *$ & $-0.238 * *$ & $-0.312 * *$ & -0.001 & -0.006 & -0.006 & -0.021 \\
\hline 83 & Ezox Natural x Ex-7959 & 0.001 & -0.001 & $-3.090 * *$ & $4.199 * *$ & $1.244^{* *}$ & $1.109^{* *}$ & 0.001 & $-0.009 *$ & 0.012 & 0.010 \\
\hline 84 & Ezox Natural x Neelam & 0.001 & -0.001 & $-1.598 * *$ & $-3.017 * *$ & $-1.005^{* *}$ & $-0.797^{* *}$ & 0.001 & $0.015^{* *}$ & -0.006 & 0.011 \\
\hline 85 & FR-3 x Heera & $0.003 * *$ & $-0.004 * *$ & $-1.540 * *$ & $14.951 * *$ & $-0.278 * *$ & $-1.172^{* *}$ & 0.0001 & $0.012 * *$ & $0.013^{*}$ & -0.023 \\
\hline 86 & FR-3 x Ex-7959 & 0.001 & 0.001 & $10.053 * *$ & $-14.638 * *$ & $-0.207 * *$ & $-0.146^{* *}$ & 0.0001 & $-0.021 * *$ & $0.013^{*}$ & 0.046 \\
\hline 87 & FR-3 x Neelam & $-0.004 * *$ & $0.003^{* *}$ & $-8.514 * *$ & $-0.312^{* *}$ & $0.485^{* *}$ & $1.318^{* *}$ & 0.0001 & $0.009 *$ & $-0.026^{* *}$ & -0.023 \\
\hline 88 & FRW-9 x Heera & $0.007 * *$ & 0.001 & $12.649 * *$ & $16.951 * *$ & $-0.598 * *$ & $-0.815^{* *}$ & 0.001 & 0.005 & $-0.022 * *$ & -0.026 \\
\hline 89 & FRW-9 x Ex-7959 & $-0.006^{* *}$ & $-0.006^{* *}$ & $-2.147 * *$ & $-6.382 * *$ & $1.190 * *$ & $0.376 * *$ & -0.002 & $0.005^{*}$ & $-0.014^{*}$ & 0.062 \\
\hline 90 & FRW-9 x Neelam & -0.001 & $0.004 * *$ & $-10.502 * *$ & $-10.569 * *$ & $-0.592 * *$ & $0.439 * *$ & 0.001 & $-0.009^{*}$ & $0.036^{* *}$ & -0.035 \\
\hline & Sij-skl & 0.001 & 0.0005 & 0.008 & 0.0007 & 0.001 & 0.005 & 0.009 & 0.004 & 0.006 & 0.061 \\
\hline
\end{tabular}

Continued.... 
Int.J.Curr.Microbiol.App.Sci (2019) 8(1): 1118-1148

\begin{tabular}{|c|c|c|c|c|c|c|c|c|c|}
\hline \multirow[t]{2}{*}{ S.No } & \multirow[t]{2}{*}{ Crosses } & \multicolumn{2}{|c|}{$\mathrm{AV}$} & \multicolumn{2}{|l|}{$\mathrm{LN}$} & \multicolumn{2}{|l|}{ LL } & \multicolumn{2}{|l|}{ IV } \\
\hline & & $\mathrm{F}_{1}$ & $\mathrm{~F}_{2}$ & $\mathrm{~F}_{1}$ & $\mathrm{~F}_{2}$ & $\mathrm{~F}_{1}$ & $\mathrm{~F}_{2}$ & $\mathrm{~F}_{1}$ & $\mathrm{~F}_{2}$ \\
\hline 1 & EC-41750 x Heera & -0.186 & -0.019 & 0.870 & $4.448 * *$ & $-2.813^{* *}$ & -0.816 & $-5.632 * *$ & $10.129 * *$ \\
\hline 2 & EC-41750 x Ex-7959 & 0.037 & $0.500 * *$ & $-10.546^{* *}$ & $4.703 * *$ & $-1.161 * *$ & $2.834 * *$ & $-2.498 * *$ & 0.091 \\
\hline 3 & EC-41750 x Neelam & 0.152 & $-0.480 * *$ & $9.677 * *$ & $-9.151^{* *}$ & $3.974 * *$ & $-2.018^{* *}$ & $8.130 * *$ & $-10.220 * *$ \\
\hline 4 & EC-41752 x Heera & -0.198 & $-0.179 * *$ & $8.657 * *$ & $5.7979 * *$ & $5.242 * *$ & $1.754 * *$ & $5.375 * *$ & $-4.535 * *$ \\
\hline 5 & EC-41752 x Ex-7959 & 0.041 & $-0.600^{* *}$ & -0.518 & $-4.370^{* *}$ & $2.906^{* *}$ & -0.217 & $-3.601 * *$ & $0.783^{* *}$ \\
\hline 6 & EC-41752 x Neelam & 0.157 & $0.827 * *$ & $-8.139 * *$ & $-1.427 * *$ & $-8.147 * *$ & $-1.538 * *$ & $-1.774 * *$ & $3.752 * *$ \\
\hline 7 & EC-98994 x Heera & 0.280 & $-0.239 * *$ & -0.612 & $-12.606^{* *}$ & $-1.802 * *$ & $-3.356^{* *}$ & $-4.167 * *$ & $-3.128 * *$ \\
\hline 8 & EC-98994 x Ex-7959 & $-0.374^{*}$ & -0.200 & $-4.058^{*}$ & $-14.179 * *$ & $-2.856^{* *}$ & $-4.387 * *$ & $-3.032 * *$ & $0.957 * *$ \\
\hline 9 & EC-98994 x Neelam & 0.094 & $0.259 * *$ & $4.670^{* *}$ & $26.785 * *$ & $4.659 * *$ & $7.743 * *$ & $7.199 * *$ & $2.170 * *$ \\
\hline 10 & T-397 x Heera & -0.232 & -0.009 & -0.064 & $6.329 * *$ & $3.345^{* * *}$ & $-6.449 * *$ & $2.968 * *$ & $-1.202 * *$ \\
\hline 11 & T-397 x Ex-7959 & -0.112 & $0.208 * *$ & $4.323 *$ & $10.466 * *$ & $7.152 * *$ & $-3.961^{* *}$ & $-1.771^{* *}$ & $5.123 * *$ \\
\hline 12 & T-397 x Neelam & $0.344^{*}$ & $-0.199 * *$ & $-4.259 *$ & $-46.795^{* *}$ & $-10.498 * *$ & $10.410^{* *}$ & $-1.197 * *$ & $-3.921 * *$ \\
\hline 13 & EC-99029 x Heera & 0.178 & $0.653 * *$ & -1.473 & $-1.208 * *$ & 0.007 & -0.249 & $-4.928 * *$ & $-3.144 * *$ \\
\hline 14 & EC-99029 x Ex-7959 & 0.124 & $-0.482 * *$ & $5.834 * *$ & $8.780 * *$ & $4.533 * *$ & $5.865 * *$ & 0.482 & $0.464 * *$ \\
\hline 15 & EC-99029 x Neelam & -0.302 & $-0.171 * *$ & $-4.361 *$ & $-7.527 * *$ & $-4.540 * *$ & $-5.616^{* *}$ & $4.447 * *$ & $2.680 * *$ \\
\hline 16 & EC-99056 x Heera & -0.034 & $0.268 * *$ & -2.607 & $-2.710 * *$ & $0.258^{*}$ & $1.307 * *$ & $-1.169 *$ & $-3.160 * *$ \\
\hline 17 & EC-99056 x Ex-7959 & 0.103 & 0.064 & $7.955^{* * *}$ & $2.759 * *$ & $-1.402 * *$ & $3.081 * *$ & $-1.174 *$ & $-0.328 * *$ \\
\hline 18 & EC-99056 x Neelam & -0.069 & $-0.332 * *$ & $-5.348 * *$ & $-0.049 * *$ & $1.144^{* *}$ & $-4.388 * *$ & $2.343 * *$ & $3.488^{* *}$ \\
\hline 19 & EC-104739 x Heera & 0.077 & $-0.150 * *$ & -0.670 & $1.710^{* *}$ & $3.416^{* * *}$ & $2.845^{* * *}$ & $1.405 * *$ & $3.836^{* *}$ \\
\hline 20 & EC-104739 x Ex-7959 & 0.190 & $-0.261 * *$ & 0.556 & $2.226 * *$ & $-1.602 * *$ & $-7.433 * *$ & $-2.461 * *$ & $-4.323 * *$ \\
\hline 21 & EC-104739 x Neelam & -0.266 & $0.411 * *$ & 0.113 & $-3.936^{* *}$ & $-1.814 * *$ & $4.588 * *$ & $1.056^{*}$ & $0.487 * *$ \\
\hline 22 & CRISTA x Heera & -0.131 & $-0.356^{* *}$ & $-7.134 * *$ & $-17.309 * *$ & $-1.865^{* *}$ & $-6.648^{* *}$ & $-3.951 * *$ & $2.337 * *$ \\
\hline 23 & CRISTA x Ex-7959 & 0.002 & $-0.290^{* *}$ & $-5.498 * *$ & $11.599 * *$ & $-1.320 * *$ & $6.619^{* *}$ & $-2.527 * *$ & $-6.531 * *$ \\
\hline 24 & CRISTA x Neelam & 0.129 & $0.647 * *$ & $12.633^{* *}$ & $5.709 * *$ & $3.185^{* *}$ & 0.030 & $6.478 * *$ & $4.195^{* *}$ \\
\hline 25 & EC-110288 x Heera & 0.222 & $0.306^{* *}$ & -2.114 & $-1.467 * *$ & $-0.687 * *$ & $4.385^{* *}$ & $-4.021 * *$ & $-4.491 * *$ \\
\hline 26 & EC-110288 x Ex-7959 & -0.042 & $-0.383^{* *}$ & $4.324 *$ & $13.702 * *$ & $-3.425 * *$ & $5.301 * *$ & $-2.663 * *$ & $7.501 * *$ \\
\hline 27 & EC-110288 x Neelam & -0.180 & 0.077 & -2.210 & $-12.235^{* *}$ & $4.112 * *$ & $-9.686 * *$ & $6.684 * *$ & $-3.010 * *$ \\
\hline 28 & EC-112689 x Heera & -0.11 & $-0.109 * *$ & -2.834 & $-1.552^{* *}$ & $-0.560 * *$ & $-8.518^{* *}$ & $-2.039 * *$ & $1.635^{* * *}$ \\
\hline 29 & EC-112689 x Ex-7959 & 0.189 & $-0.166^{* *}$ & 2.494 & $18.381 * *$ & $1.334 * *$ & $3.158 * *$ & 0.872 & $7.443^{* *}$ \\
\hline 30 & EC-112689 x Neelam & -0.177 & $0.275 * *$ & 0.340 & $-16.829 * *$ & $-0.773 * *$ & $5.359 * *$ & $1.166^{*}$ & $-9.078 * *$ \\
\hline 31 & EC-115161 x Heera & 0.237 & $0.233^{* *}$ & $-4.867 *$ & $0.802 * *$ & $-0.228 *$ & $3.929 * *$ & $-2.199 * *$ & $0.081^{* *}$ \\
\hline 32 & EC-115161 x Ex-7959 & -0.172 & $-0.469 * *$ & 2.194 & $-4.518 * *$ & $3.616^{* *}$ & $-1.444 * *$ & -0.214 & $-2.977 * *$ \\
\hline 33 & EC-115161 x Neelam & -0.065 & $0.237 * *$ & 2.673 & $3.716^{* *}$ & $-3.388 * *$ & $-2.485^{* *}$ & $2.413 * *$ & $2.896 * *$ \\
\hline 34 & EC-115162 x Heera & -0.114 & $0.883 * *$ & 3.445 & $-13.441 * *$ & $1.963 * *$ & $-8.497 * *$ & $-5.669 * *$ & $-3.438 * *$ \\
\hline 35 & EC-115162 x Ex-7959 & 0.095 & $-0.560 * *$ & 0.786 & $6.252 * *$ & $1.884 * *$ & $3.547 * *$ & $3.576 * *$ & $0.704 * *$ \\
\hline 36 & EC-115162 x Neelam & 0.018 & $-0.323^{* *}$ & $-4.231 *$ & $7.189^{* *}$ & $-3.847 * *$ & $4.950 * *$ & $2.093 * *$ & $2.734 * *$ \\
\hline 37 & EC-115178 x Heera & -0.076 & $-0.102 *$ & 2.139 & $2.265^{* *}$ & $-2.376 * *$ & $-1.531 * *$ & $-3.451 * *$ & $2.750 * *$ \\
\hline 38 & EC-115178 x Ex-7959 & -0.182 & $-0.203 * *$ & $-7.871^{* *}$ & $0.554 * *$ & $7.796 * *$ & $-2.616^{* *}$ & $-1.650 * *$ & $1.195 * *$ \\
\hline 39 & EC-115178 x Neelam & 0.258 & $0.305 * *$ & $5.732 * *$ & $-2.819 * *$ & $-5.419 * *$ & $4.147 * *$ & $5.102 * *$ & $-3.945 * *$ \\
\hline 40 & EC-115186 x Heera & -0.217 & $-0.252 * *$ & $6.020^{* *}$ & $-0.960 * *$ & $3.987 * *$ & $2.443 * *$ & $-1.705 * *$ & $-2.479 * *$ \\
\hline 41 & EC-115186 x Ex-7959 & 0.219 & $0.206 * *$ & $-10.409 * *$ & $-12.387 * *$ & $-6.842 * *$ & $-6.966^{* *}$ & $-4.794 * *$ & $3.833 * *$ \\
\hline 42 & EC-115186 x Neelam & -0.002 & 0.046 & $4.388^{*}$ & $13.347 * *$ & $2.855^{* *}$ & $4.523 * *$ & $6.500 * *$ & $-1.354 * *$ \\
\hline 43 & EC-225125 x Heera & -0.086 & $-0.557 * *$ & -1.618 & $10.016^{* *}$ & $4.802 * *$ & $8.129 * *$ & $-4.736^{* *}$ & $-1.610 * *$ \\
\hline 44 & EC-225125 x Ex-7959 & -0.156 & $-0.563^{* *}$ & -2.055 & $-0.267 * *$ & $4.396^{* *}$ & $-1.899 * *$ & -0.667 & $2.452 * *$ \\
\hline 45 & EC-225125 x Neelam & 0.242 & $1.119 * *$ & 3.673 & $-9.749 * *$ & $-9.198 * *$ & $-6.230 * *$ & $5.403 * *$ & $-0.842 * *$ \\
\hline
\end{tabular}

Continued.... 
Int.J.Curr.Microbiol.App.Sci (2019) 8(1): 1118-1148

\begin{tabular}{|c|c|c|c|c|c|c|c|c|c|}
\hline \multirow{2}{*}{ S.No } & \multirow{2}{*}{ Crosses } & \multicolumn{2}{|c|}{$\mathrm{AV}$} & \multicolumn{2}{|l|}{$\mathrm{LN}$} & \multicolumn{2}{|l|}{ LL } & \multicolumn{2}{|l|}{ IV } \\
\hline & & $\mathrm{F}_{1}$ & $F_{2}$ & $\mathrm{~F}_{1}$ & $F_{2}$ & $\mathrm{~F}_{1}$ & $\mathrm{~F}_{2}$ & $\mathrm{~F}_{1}$ & $\mathrm{~F}_{2}$ \\
\hline 46 & EC-1041492 x Heera & 0.043 & $0.111 * *$ & $-5.193 * *$ & $-5.175^{* *}$ & $6.023 * *$ & $-14.696 * *$ & $-1.558 * *$ & $-3.279 * *$ \\
\hline 47 & EC-1041492 x Ex-7959 & 0.003 & $0.758 * *$ & 0.670 & $10.266^{* *}$ & $-8.804 * *$ & $1.295 * *$ & $-4.980 * *$ & $-0.264^{*}$ \\
\hline 48 & EC-1041492 x Neelam & -0.046 & $-0.869 * *$ & $4.524 *$ & $-5.091 * *$ & $2.781 * *$ & $13.400 * *$ & $6.537 * *$ & $3.542^{* *}$ \\
\hline 49 & Mukta x Heera & 0.075 & $0.211^{* *}$ & $-4.732 *$ & $-10.555^{* *}$ & $7.807 * *$ & $-1.553 * *$ & -0.309 & $-6.970 * *$ \\
\hline 50 & Mukta x Ex-7959 & -0.242 & $0.083^{*}$ & $-4.201 *$ & $2.283 * *$ & $-9.871 * *$ & $3.324 * *$ & $-9.144 * *$ & $-0.855^{* *}$ \\
\hline 51 & Mukta x Neelam & 0.167 & $-0.294 * *$ & $8.933 * *$ & $8.272 * *$ & $2.065 * *$ & $-1.771 * *$ & $9.453 * *$ & $7.825^{* *}$ \\
\hline 52 & ES-1463 x Heera & -0.080 & $-1.061 * *$ & 1.005 & $2.074 * *$ & $-6.393 * *$ & $9.131 * *$ & 5.378 & $10.372 * *$ \\
\hline 53 & ES-1463 x Ex-7959 & 0.319 & $0.472 * *$ & 1.944 & $-10.262 * *$ & $4.029 * *$ & $-10.227 * *$ & $-1.518 * *$ & $-3.176^{* *}$ \\
\hline 54 & ES-1463 x Neelam & -0.239 & $0.589 * *$ & -2.949 & $8.188^{* *}$ & $2.364 * *$ & $1.096^{*}$ & $-3.860 * *$ & $-7.196^{* *}$ \\
\hline 55 & ES-1474 x Heera & 0.060 & $-0.340 * *$ & 0.770 & $-6.850 * *$ & $3.283^{* *}$ & $-7.783 * *$ & $-1.955^{* *}$ & $-5.972 * *$ \\
\hline 56 & ES-1474 x Ex-7959 & -0.269 & -0.004 & -0.875 & $5.806^{* *}$ & $-3.793 * *$ & $0.719^{*}$ & -0.851 & $4.493^{* *}$ \\
\hline 57 & ES-1474 x Neelam & 0.209 & $0.344 * *$ & 0.106 & $1.044 * *$ & $0.510^{* *}$ & $7.065^{* *}$ & $2.806 * *$ & $1.479^{* *}$ \\
\hline 58 & ES-1496 x Heera & -0.119 & $0.088^{*}$ & $-3.773^{*}$ & $5.548 * *$ & $-2.780 * *$ & $1.393 * *$ & $-1.743^{* *}$ & $2.388^{* *}$ \\
\hline 59 & ES-1496 x Ex-7959 & 0.313 & $0.542 * *$ & 2.101 & $9.161 * *$ & $2.147 * *$ & $8.391 * *$ & $-2.275^{* *}$ & $1.396^{* *}$ \\
\hline 60 & ES-1496 x Neelam & -0.194 & $-0.630 * *$ & 1.672 & $-14.710 * *$ & $0.634 * *$ & $-9.784 * *$ & $4.019 * *$ & $-3.784 * *$ \\
\hline 61 & ES-1531 x Heera & 0.098 & $-0.176^{* *}$ & 1.954 & $-5.390 * *$ & $2.160 * *$ & -0.198 & $-5.743^{* *}$ & $-4.564 * *$ \\
\hline 62 & ES-1531 x Ex-7959 & -0.210 & 0.012 & 3.019 & $-12.343^{* *}$ & $3.674 * *$ & $-10.535^{* *}$ & $-1.609^{* *}$ & $-4.643 * *$ \\
\hline 63 & ES-1531 x Neelam & 0.112 & $0.164 * *$ & $-4.973 * *$ & $17.732 * *$ & $-5.834 * *$ & $10.734 * *$ & $7.352 * *$ & $9.207 * *$ \\
\hline 64 & ES-14600 x Heera & -0.119 & $-0.492 * *$ & $4.200 *$ & $28.635^{* *} *$ & $3.052 * *$ & $4.532 * *$ & $2.411^{* *}$ & $10.945^{* *}$ \\
\hline 65 & ES-14600 x Ex-7959 & -0.180 & $0.364 * *$ & -1.063 & $-10.073 * *$ & $-2.153 * *$ & $-1.043^{*}$ & $-3.584 * *$ & $-5.220 * *$ \\
\hline 66 & ES-14600 x Neelam & 0.299 & $0.129 * *$ & -3.137 & $-18.562 * *$ & $-0.899 * *$ & $-3.489 * *$ & $1.173^{*}$ & $-5.724 * *$ \\
\hline 67 & Ex-3 x Heera & -0.050 & $0.140 * *$ & $-4.933^{*}$ & $-11.613^{* *}$ & $-3.886^{* *}$ & $2.060^{* *}$ & $-4.568^{* *}$ & $-9.219 * *$ \\
\hline 68 & Ex-3 x Ex-7959 & 0.245 & $-0.100 *$ & -0.021 & $-0.630 * *$ & $9.155^{* *}$ & $1.042 *$ & $-9.670 * *$ & $5.210^{* *}$ \\
\hline 69 & Ex-3 x Neelam & -0.196 & -0.041 & 4.954* & $12.542 * *$ & $-5.269 * *$ & $-3.102 * *$ & $14.237 * *$ & $4.009 * *$ \\
\hline 70 & Ex-5-36E x Heera & 0.283 & $0.952 * *$ & $12.828 * *$ & $5.705 * *$ & $-0.843 * *$ & $3.211^{* *}$ & $-2.959 * *$ & $1.182^{* *}$ \\
\hline 71 & Ex-5-36E x Ex-7959 & -0.147 & $-0.591 * *$ & $-8.662 * *$ & $-7.068^{* *}$ & $-1.504 * *$ & $-2.686^{* *}$ & $-3.974 * *$ & $-7.023 * *$ \\
\hline 72 & Ex-5-36E x Neelam & -0.136 & $-0.362 * *$ & $-4.166^{*}$ & $1.363 * *$ & $2.347 * *$ & -0.525 & $6.933 * *$ & $5.840 * *$ \\
\hline 73 & Ex-28-3 x Heera & 0.219 & $-0.161 * *$ & 3.331 & $-5.194 * *$ & $-4.408 * *$ & $-9.452 * *$ & $60.801^{* *}$ & $-1.572 * *$ \\
\hline 74 & Ex-28-3 x Ex-7959 & -0.189 & $0.961 * *$ & 2.914 & $-0.899 * *$ & $-6.373 * *$ & $16.436^{* *}$ & $62.452 * *$ & $-0.614 * *$ \\
\hline 75 & Ex-28-3 x Neelam & -0.030 & $-0.800^{* *}$ & $-6.245^{* *}$ & $6.093^{* *}$ & $10.781^{* * *}$ & $-6.983 * *$ & $-123.254 * *$ & $2.186^{* *}$ \\
\hline 76 & Ex-313-23 x Heera & 0.101 & $0.576^{* *}$ & $-6.643 * *$ & $16.020 * *$ & $-3.375^{* *}$ & $10.310^{* *}$ & $-10.162 * *$ & $5.184^{* *}$ \\
\hline 77 & Ex-313-23 x Ex-7959 & -0.005 & $-0.306^{* *}$ & $12.043 * *$ & $-8.804 * *$ & -0.092 & $-4.737 * *$ & 0.176 & $-1.495 * *$ \\
\hline 78 & Ex-313-23 x Neelam & -0.096 & $-0.270 * *$ & $-5.400 * *$ & $-7.216^{* *}$ & $3.468 * *$ & $-5.293 * *$ & $9.986 * *$ & $-3.689 * *$ \\
\hline 79 & Ex-339-6 x Heera & 0.011 & $-0.417 * *$ & $10.263^{* *}$ & $5.511 * *$ & $1.806^{* *}$ & $2.663 * *$ & $4.949 * *$ & $-0.839 * *$ \\
\hline 80 & Ex-339-6 x Ex-7959 & 0.170 & $0.916^{* *}$ & $-5.043 * *$ & $-6.926^{* *}$ & $-4.571 * *$ & $2.497 * *$ & $-5.197 * *$ & $2.490 * *$ \\
\hline 81 & Ex-339-6 x Neelam & -0.181 & $-0.499 * *$ & $-5.220 * *$ & $1.415^{* *}$ & $2.765^{* *}$ & $-5.160 * *$ & 0.247 & $-1.651 * *$ \\
\hline 82 & Ezox Natural x Heera & -0.321 & $0.090 *$ & -3.630 & $8.721 * *$ & $-9.124 * *$ & $20.770^{* *}$ & $-10.255^{* *}$ & $11.526^{* *}$ \\
\hline 83 & Ezox Natural x Ex-7959 & 0.121 & -0.013 & $6.186 * *$ & $-11.448 * *$ & $5.599 * *$ & $-8.643 * *$ & $7.879 * *$ & $-6.873 * *$ \\
\hline 84 & Ezox Natural x Neelam & 0.200 & -0.077 & -2.555 & $2.727 * *$ & $3.525 * *$ & $-12.127 * *$ & $2.376^{* *}$ & $-4.653 * *$ \\
\hline 85 & FR-3 x Heera & 0.070 & $0.261 * *$ & $4.028 *$ & $-7.355^{* *}$ & $-0.853 * *$ & $-4.737 * *$ & $2.221 * *$ & $-7.136 * *$ \\
\hline 86 & FR-3 x Ex-7959 & 0.138 & $-0.171 * *$ & $-6.624 * *$ & $-2.463 * *$ & $-0.804 * *$ & $-7.319 * *$ & $-3.591 * *$ & $-2.641 * *$ \\
\hline 87 & FR-3 x Neelam & -0.208 & $-0.090 *$ & 2.236 & $9.818 * *$ & $1.657 * *$ & $12.056^{* *}$ & $1.370^{* * *}$ & $9.778 * *$ \\
\hline 88 & FRW-9 $\times$ Heera & 0.024 & $-0.152 * *$ & $-6.612 * *$ & $0.103 * *$ & $-5.156 * *$ & $-4.100 * *$ & $-2.589 * *$ & $4.372 * *$ \\
\hline 89 & FRW-9 x Ex-7959 & -0.028 & $0.145^{* *}$ & $9.742 * *$ & $-0.300 * *$ & $-1.646^{* *}$ & $10.005 * *$ & $-1.991 * *$ & $2.827 * *$ \\
\hline 90 & FRW-9 x Neelam & 0.004 & 0.007 & -3.131 & 0.197 & $6.802 * *$ & $-5.905 * *$ & $4.580 * *$ & $-7.200^{* *}$ \\
\hline & Sij-skl & 0.162 & 0.039 & 1.903 & 0.0004 & 0.128 & 0.423 & 0.452 & 0.110 \\
\hline
\end{tabular}

$\mathrm{OIL}=$ Oil $(\%), \mathrm{BD}=$ Bulk density $\left(\mathrm{g} / \mathrm{cm}^{3}\right), \mathrm{TD}=$ True density $\left(\mathrm{g} / \mathrm{cm}^{3}\right), \mathrm{PO}=\mathrm{Porosity}(\%), \mathrm{PR}=\mathrm{Protein}(\%), \mathrm{FAA}=$

Free amino acids $(\mathrm{g} / \mathrm{Kg}), \mathrm{CAR}=$ Carbohydrate $(\%), \mathrm{PHE}=\mathrm{Phenol}(\%), \mathrm{RF}=$ Refractive index, $\mathrm{SG}=$ Specific gravity, $\mathrm{AV}=$ Acid value, $\mathrm{LN}=$ Linolenic acid $(\%), \mathrm{LL}=$ Linoleic acid $(\%)$ and $\mathrm{IV}=$ Iodine value. 
Table.3 Ranking of parents (lines and testers) on the basis of per se performance and gca effect for all traits in both generations

\begin{tabular}{|c|c|c|c|c|}
\hline \multicolumn{2}{|c|}{ Traits } & \multirow{3}{*}{$\begin{array}{r}\text { per se performance } \\
\text { Heera, Neelam, Ex-7959 }\end{array}$} & \multicolumn{2}{|c|}{ Good General combiners } \\
\hline & & & \multirow{2}{*}{$\begin{array}{l}\text { Feneration } \\
\text { Neelam }\end{array}$} & \multirow{2}{*}{ None $\quad F_{2}$ generation } \\
\hline Oil percentage & Testers & & & \\
\hline & Lines & $\begin{array}{l}\text { EC-41752, ES-1496, EC- } \\
\text { 104739, EC-115161, Ex-313-23 }\end{array}$ & $\begin{array}{l}\text { Ex-313-23, EC- } \\
110288, \text { EC-115161, } \\
\text { EC-115178, EC- } \\
112689\end{array}$ & $\begin{array}{l}\text { CRISTA, Ex-5-36E, Ex-28-3, ES-1474, } \\
\text { FRW-9, EC-41752, EC-112689, Ezox } \\
\text { Natural }\end{array}$ \\
\hline \multirow[t]{2}{*}{ Bulk density } & Testers & Neelam, Heera, Ex-7959 & None & None \\
\hline & Lines & $\begin{array}{l}\text { Ex-313-23, Ex-5-36E, EC- } \\
\text { 98994, EC-112689, FRW-9 }\end{array}$ & $\begin{array}{l}\text { EC-41752, EC- } \\
1041492, \text { Ex-3, Ex-28- } \\
3, \text { EC-225125 }\end{array}$ & $\begin{array}{l}\text { Ex-3, Ezox Natural, Ex-339-6, Ex-28-3, } \\
\text { ES-1474 }\end{array}$ \\
\hline \multirow[t]{2}{*}{ True density } & Testers & Neelam, Heera, Ex-7959 & Heera & Heera \\
\hline & Lines & $\begin{array}{l}\text { EC-1041492, EC-225125, Ex-3, } \\
\text { Ex-313-23, Ex-339-6 }\end{array}$ & $\begin{array}{l}\text { EC-41752, EC-41750, } \\
\text { EC-1041492, Ex-3, } \\
\text { Ex-28-3 }\end{array}$ & $\begin{array}{l}\text { FR-3, EC-99056, EC-104739, EC- } \\
99056, \text { EC-41752 }\end{array}$ \\
\hline \multirow[t]{2}{*}{ Porosity } & Testers & Heera, Ex-7959, Neelam & Ex-7959 & Ex-7959 \\
\hline & Lines & $\begin{array}{l}\text { ES-1496, Ex-28-3, EC-98994, } \\
\text { FR-3, ES-14600 }\end{array}$ & $\begin{array}{l}\text { ES-14600, EC-98994, } \\
\text { ES-1474, EC-99029, } \\
\text { Ex-313-23 }\end{array}$ & $\begin{array}{l}\text { EC-115161, EC-112689, Ex-313-23, } \\
\text { Ex-339-6, Ex-28-3 }\end{array}$ \\
\hline \multirow[t]{2}{*}{ Protein } & Testers & Ex-7959, Neelam, Heera & None & Heera, Neelam \\
\hline & Lines & $\begin{array}{l}\text { T-397, EC-99029, EC-115186, } \\
\text { Ex-339-6, EC-112689 }\end{array}$ & $\begin{array}{l}\text { ES-1463, EC-41752, } \\
\text { Ezox Natural, ES- } \\
1474, \text { EC-41750 }\end{array}$ & $\begin{array}{l}\text { T-397, ES-1496, Ex-110288, Ex-5-36E, } \\
\text { ES-14600 }\end{array}$ \\
\hline \multirow[t]{2}{*}{ Free amino acids } & Testers & Ex-7959, Heera, Neelam & None & None \\
\hline & Lines & $\begin{array}{l}\text { ES-1463, Ex-28-3, Mukta, EC- } \\
\text { 104739, FRW-9 }\end{array}$ & $\begin{array}{l}\text { EC-115162, EC- } \\
112689, \text { Mukta, EC- } \\
225125, \text { EC-115178, } \\
\text { EC-115161 }\end{array}$ & $\begin{array}{l}\text { T-397, ES-1496, EC-110288, Ex-5- } \\
\text { 36E, ES-14600, EC-115162 }\end{array}$ \\
\hline \multirow[t]{2}{*}{ Carbohydrate } & Testers & Ex-7959, Heera, Neelam & Ex-7959 & Heera \\
\hline & Lines & $\begin{array}{l}\text { ES-1531, EC-41752, Ex-3, EC- } \\
\text { 98994, EC-112689 }\end{array}$ & $\begin{array}{l}\text { EC-115161, ES -1463, } \\
\text { Ex-28-3, EC-115162, } \\
\text { EC-104739 }\end{array}$ & $\begin{array}{l}\text { EC-225125, EC-99029, EC-115186, } \\
\text { EC-99056, Ezox Natural }\end{array}$ \\
\hline \multirow[t]{2}{*}{ Phenol } & Testers & Heera, Neelam, Ex-7959 & Ex-7959 & Ex-7959 \\
\hline & Lines & $\begin{array}{l}\text { Ex-3, EC-115178, EC-1041492, } \\
\text { EC-99029, ES-1531 }\end{array}$ & $\begin{array}{l}\text { Ex-5-36E, CRISTA, } \\
\text { Ex-28-3, EC-99056, } \\
\text { Ex-339-6 }\end{array}$ & $\begin{array}{l}\text { Ex-5-36E, T-397, CRISTA, EC-41750, } \\
\text { EC-1041492 }\end{array}$ \\
\hline \multirow[t]{2}{*}{ Refractive index } & Testers & Neelam, Ex-7959, Heera & None & None \\
\hline & Lines & $\begin{array}{l}\text { Mukta, ES-14600, EC-41752, } \\
\text { EC-41750, T-397, Ex-313-23 }\end{array}$ & $\begin{array}{l}\text { EC-104739, EC- } \\
115178, \text { EC-112689, } \\
\text { CRISTA, EC-99056 }\end{array}$ & $\begin{array}{l}\text { Ex-5-36E, FR-3, CRISTA, FRW-9, ES- } \\
1531\end{array}$ \\
\hline \multirow[t]{2}{*}{ Specific gravity } & Testers & Ex-7959, Heera, Neelam & None & None \\
\hline & Lines & $\begin{array}{l}\text { Ex-28-3, Ezox Natural, EC- } \\
\text { 112689, CRISTA, EC-115186 }\end{array}$ & $\begin{array}{l}\text { FR-3, Ezox Natural, } \\
\text { Ex-339-6, EC-99029, } \\
\text { EC-225125 }\end{array}$ & $\begin{array}{l}\text { Ex-339-6, EC-112689, Mukta, EC- } \\
115186, \text { EC-225125 }\end{array}$ \\
\hline \multirow[t]{2}{*}{ Acid value } & Testers & Heera, Ex-7959, Neelam & None & Heera \\
\hline & Lines & $\begin{array}{l}\text { Ezox Natural, EC-1041492, Ex- } \\
\text { 28-3, ES-1496, Ex-5-36E }\end{array}$ & $\begin{array}{l}\text { EC-104739, ES-14600, } \\
\text { EC-41750, EC- } \\
115186, \text { Ex-3 }\end{array}$ & $\begin{array}{l}\text { EC-112689, EC-104739, T-397, FRW- } \\
\text { 9, Mukta }\end{array}$ \\
\hline \multirow[t]{2}{*}{ Linolenic acid } & Testers & Ex-7959, Neelam, Heera & Neelam & Neelam \\
\hline & Lines & $\begin{array}{l}\text { ES-1463, ES-14600, Mukta, EC- } \\
\text { 1041492, Ex-313-23 }\end{array}$ & $\begin{array}{l}\text { Ex-5-36E, CRISTA, } \\
\text { EC-41750, EC-99029, } \\
\text { ES-1496 }\end{array}$ & $\begin{array}{l}\text { EC-110288, EC-98994, Ex-5-36E, } \\
\text { Ezox Natural, Ex-28-3 }\end{array}$ \\
\hline \multirow[t]{2}{*}{ Linoleic acid } & Testers & Neelam, Heera, Ex-7959 & Neelam & Neelam \\
\hline & Lines & $\begin{array}{l}\text { ES-1496, EC-110288, EC- } \\
\text { 98994, EC-115178, FR-3 }\end{array}$ & $\begin{array}{l}\text { EC-112689, EC- } \\
104739, \text { FRW-9, EC- } \\
115162, \text { EC-99056 }\end{array}$ & $\begin{array}{l}\text { EC-115178, ES-1474, EC-112689, EC- } \\
\text { 104739, Ex-28-3 }\end{array}$ \\
\hline \multirow[t]{2}{*}{ Iodine value } & Testers & Heera, Ex-7959, Neelam & Neelam & Neelam \\
\hline & Lines & $\begin{array}{l}\text { Mukta, EC-99029, EC-1041492, } \\
\text { EC-99056, Ex-5-36E, FR-3 }\end{array}$ & $\begin{array}{l}\text { Ex-28-3, CRISTA, Ex- } \\
\text { 5-36E, ES-1496, FR-3 }\end{array}$ & $\begin{array}{l}\text { EC-110288, EC-98994, Ex-5-36E, } \\
\text { Ezox Natural, Ex-28-3 }\end{array}$ \\
\hline
\end{tabular}


Table.4 Grading of crosses on the basis of per se performance and sca effect in both generations for all traits in linseed

\begin{tabular}{|c|c|c|c|c|c|}
\hline \multirow[t]{2}{*}{ Traits } & \multicolumn{2}{|r|}{ per se performance } & \multicolumn{3}{|c|}{ Good Specific combiners } \\
\hline & $\mathrm{F}_{1}$ generation & $\mathrm{F}_{2}$ generation & $\mathrm{F}_{1}$ generation & $\mathrm{F}_{2}$ generation & $\begin{array}{l}\text { In both } \\
\text { generations }\end{array}$ \\
\hline OIL & $\begin{array}{l}\text { EC-110288 x } \\
\text { Neelam, Ex-3 x Ex- } \\
7959, \text { EC-112689 x } \\
\text { Neelam, ES-1463 x } \\
\text { Neelam, EC-115178 } \\
\text { x Heera }\end{array}$ & $\begin{array}{l}\text { CRISTA x Neelam, EC-115162 x Neelam, } \\
\text { Ex-5-36E x Ex-7959, ES-1474 x Heera, EC- } \\
41752 \text { x Ex-7959 }\end{array}$ & $\begin{array}{l}\text { Ex-3 x Ex-7959, } \\
\text { ES-1463 x } \\
\text { Neelam, EC- } \\
110288 \text { x Neelam, } \\
\text { EC-115186 x } \\
\text { Heera, EC-104739 } \\
\text { x Heera }\end{array}$ & $\begin{array}{l}\text { EC-115162 x } \\
\text { Neelam, EC-115161 } \\
\text { x Ex-7959, ES-1474 } \\
\text { x Heera, Ezox } \\
\text { Natural x Heera, } \\
\text { EC-41752 x Ex- } \\
7959\end{array}$ & - \\
\hline BD & $\begin{array}{l}\text { Ex-28-3 x Heera, } \\
\text { EC-41752 x Ex- } \\
7959, \text { Ex-313-23 x } \\
\text { Ex-7959, ES-1463 x } \\
\text { Neelam, EC-98994 x } \\
\text { Heera }\end{array}$ & $\begin{array}{l}\text { EC-115186 x Neelam, T-397 x Heera, ES- } \\
1496 \text { x Ex-7959, Ezox Natural x Neelam, Ex- } \\
\text { 339-6 x Heera }\end{array}$ & $\begin{array}{l}\text { ES-1463 x } \\
\text { Neelam, EC- } \\
115186 \text { x Ex-7959, } \\
\text { EC-115178 x } \\
\text { Heera, EC-98994 x } \\
\text { Heera, Ex-28-3 x } \\
\text { Heera }\end{array}$ & $\begin{array}{l}\text { T-397 x Heera, EC- } \\
115186 \times \text { Neelam, } \\
\text { ES-1496 x Ex-7959, } \\
\text { ES-1463 x Heera, } \\
\text { EC-41750 x Ex- } \\
7959\end{array}$ & - \\
\hline TD & $\begin{array}{l}\text { EC-41750 x Heera, } \\
\text { Ex-28-3 x Heera, } \\
\text { EC-41752 x Ex- } \\
7959, \text { Ex-313-23 x } \\
\text { Ex-7959, ES-1463 x } \\
\text { Neelam }\end{array}$ & $\begin{array}{l}\text { EC-41752 x Ex-7959, EC-41752 x Neelam, } \\
\text { EC-99056 x Heera, EC-99056 x Neelam, EC- } \\
104739 \text { x Heera,, EC-104739 x Neelam, } \\
\text { CRISTA x Heera, CRISTA x Neelam, EC- } \\
110288 \text { x Heera, EC-115186 x Neelam, EC- } \\
225125 \times \text { Heera, ES-1474 x Heera, ES-1531 } \\
\text { x Heera, Ex-3 x Heera, Ex-5-36E x Heera, } \\
\text { FR-3 x Heera, FR-3 x Ex-7959, FR-3 x } \\
\text { Neelam, FRW-9 x Heera }\end{array}$ & $\begin{array}{l}\text { ES-1463 x } \\
\text { Neelam, EC- } \\
115186 \text { x Ex-7959, } \\
\text { Ex-313-23 x Ex- } \\
7959, \text { EC-115178 } \\
\text { x Heera, Ex-28-3 x } \\
\text { Heera }\end{array}$ & $\begin{array}{l}\text { EC-115186 x } \\
\text { Neelam, EC-110288 } \\
\text { x Heera, EC-115178 } \\
\text { x Heera, Ex-3 x } \\
\text { Heera, Ex-5-36E x } \\
\text { Heera }\end{array}$ & $\begin{array}{l}\text { EC-115178 x } \\
\text { Heera }\end{array}$ \\
\hline PO & $\begin{array}{l}\text { EC-115186 x } \\
\text { Neelam, ES-14600 x } \\
\text { Neelam, EC-104739 } \\
\text { x Ex-7959, ES-1474 } \\
\text { x Neelam, EC-98994 } \\
\text { x Neelam }\end{array}$ & $\begin{array}{l}\text { Ex-313-23 x Ex-7959, Ex-3 x Ex-7959, Ex- } \\
28-3 \text { x Neelam, Ex-339-6 x Ex-7959, EC- } \\
110288 \text { x Neelam }\end{array}$ & $\begin{array}{l}\text { EC-115186 x } \\
\text { Neelam, Ex-28-3 x } \\
\text { Heera, EC-104739 } \\
\text { x Ex-7959, FRW-9 } \\
\text { x Ex-7959, EC- } \\
115178 \text { x Heera, }\end{array}$ & $\begin{array}{l}\text { EC-41752 x Heera, } \\
\text { Ex-5-36E x Neelam, } \\
\text { Ex-28-3 x Neelam, } \\
\text { Ex-3 x Ex-7959, } \\
\text { EC-110288 x } \\
\text { Neelam }\end{array}$ & - \\
\hline PR & $\begin{array}{l}\text { Ezox Natural x } \\
\text { Heera, EC-104739 x } \\
\text { Heera, ES-1463 x } \\
\text { Ex-7959, ES-14600 } \\
\text { x Neelam, Mukta x } \\
\text { Neelam }\end{array}$ & $\begin{array}{l}\text { ES-1496 x Neelam, ES-1531 x Neelam, T- } \\
397 \text { x Ex-7959, T-397 x Heera, EC-98994 x } \\
\text { Heera }\end{array}$ & $\begin{array}{l}\text { Ezox Natural x } \\
\text { Heera, EC-104739 } \\
\text { x Heera, EC- } \\
1041492 \text { x Ex- } \\
7959, \text { Mukta x } \\
\text { Neelam, T-397 x } \\
\text { Neelam }\end{array}$ & $\begin{array}{l}\text { ES-1531 x Neelam, } \\
\text { EC-115161 x Heera, } \\
\text { EC-41752 x Ex- } \\
7959, \text { EC-98994 x } \\
\text { Heera, EC-99056 x } \\
\text { Ex-7959 }\end{array}$ & - \\
\hline FAA & $\begin{array}{l}\text { EC-115162 x Heera, } \\
\text { EC-115162 x Ex- } \\
7959, \text { EC-41752 x } \\
\text { Heera, EC-112689 x } \\
\text { Heera, EC-115186 x } \\
\text { Heera, EC-225125 x } \\
\text { Ex-7959, ES-1474 x } \\
\text { Neelam }\end{array}$ & $\begin{array}{l}\text { ES-1496 x Neelam, ES-1531 x Neelam, T- } \\
397 \text { x Ex-7959, T-397 x Heera, EC-98994 x } \\
\text { Heera, EC-110288 x Heera, EC-110288 x } \\
\text { Neelam, EC-115161 x Heera, ES-14600 x } \\
\text { Neelam, EC-41752 x Ex-7959 }\end{array}$ & $\begin{array}{l}\text { EC-99056 x Ex- } \\
\text { 7959, FRW-9 x } \\
\text { Heera, ES-1474 x } \\
\text { Neelam,, EC- } \\
104739 \text { x Neelam, } \\
\text { T-397 x Heera, } \\
\text { EC-115186 x } \\
\text { Heera, Ex-3 x } \\
\text { Heera }\end{array}$ & $\begin{array}{l}\text { EC-115161 x Heera, } \\
\text { EC-98994 x Heera, } \\
\text { EC-41752 x Ex- } \\
\text { 7959, EC-99056 x } \\
\text { Ex-7959, CRISTA x } \\
\text { Heera, EC-115161 x } \\
\text { Ex-7959, EC- } \\
115178 \text { x Neelam, } \\
\text { EC-1041492 x } \\
\text { Neelam, ES-1463 x } \\
\text { Neelam, ES-1496 x } \\
\text { Neelam }\end{array}$ & $\begin{array}{l}\text { EC-99056 x } \\
\text { Ex-7959 }\end{array}$ \\
\hline CAR & $\begin{array}{l}\text { ES-1531 x Heera, } \\
\text { EC-225125 x Ex- } \\
7959, \text { Ex-5-36E x } \\
\text { Neelam, EC-115161 } \\
\text { x Ex-7959, EC- } \\
104739 \text { x Neelam }\end{array}$ & $\begin{array}{l}\text { ES-1496 x Ex-7959, FRW-9 x Heera, EC- } \\
\text { 99056 x Ex-7959, FR-3 x Heera, T-397 x } \\
\text { Neelam }\end{array}$ & $\begin{array}{l}\text { ES-1531 x Heera, } \\
\text { FRW-9 x Heera, } \\
\text { CRISTA x } \\
\text { Neelam, Ex-313- } \\
23 \text { x Ex-7959, FR- } \\
3 \times \text { Ex-7959 }\end{array}$ & $\begin{array}{l}\text { EC-110288 x } \\
\text { Neelam, ES-1496 x } \\
\text { Ex-7959, FRW-9 x } \\
\text { Heera, FR-3 x } \\
\text { Heera, T-397 x } \\
\text { Neelam }\end{array}$ & $\begin{array}{l}\text { FRW-9 x } \\
\text { Heera }\end{array}$ \\
\hline
\end{tabular}




\begin{tabular}{|c|c|c|c|c|c|}
\hline PHE & $\begin{array}{l}\text { EC-110288 x Heera, } \\
\text { Ex-5-36E x Neelam, } \\
\text { Ex-5-36E x Ex- } \\
7959, \text { Ezox Natural } \\
\text { x Ex-7959, Ex-313- } \\
23 \text { x Heera, EC- } \\
1041492 \text { x Neelam }\end{array}$ & $\begin{array}{l}\text { EC-41750 x Ex-7959, T-397 x Ex-7959, EC- } \\
99056 \text { x Ex-7959, T-397 x Heera, EC-110288 } \\
\text { x Heera, Ex-339-6 x Ex-7959 }\end{array}$ & $\begin{array}{l}\text { EC-115162 x } \\
\text { Neelam, EC- } \\
110288 \text { x Heera, } \\
\text { EC-112689 x } \\
\text { Heera, Ex-313-23 } \\
\text { x Heera, EC-98994 } \\
\text { x Ex-7959 }\end{array}$ & $\begin{array}{l}\text { EC-41750 x Ex- } \\
7959, \text { EC-110288 x } \\
\text { Heera, EC-99056 x } \\
\text { Ex-7959, EC- } \\
115186 \text { x Heera, } \\
\text { EC-104739 x Heera }\end{array}$ & $\begin{array}{l}\text { EC-110288 x } \\
\text { Heera }\end{array}$ \\
\hline RF & $\begin{array}{l}\text { EC-104739 x Ex- } \\
7959, \text { EC-99056 x } \\
\text { Ex-7959, EC- } \\
115162 \text { x Heera, } \\
\text { EC-115186 x Ex- } \\
7959, \text { EC-225125 x } \\
\text { Neelam, EC-115178 } \\
\text { x Ex-7959, EC- } \\
104739 \text { x Heera, } \\
\text { CRISTA x Neelam, } \\
\text { EC-110288 x } \\
\text { Neelam, EC-112689 } \\
x \text { Neelam }\end{array}$ & $\begin{array}{l}\text { EC-112689 x Neelam, ES-1474 x Heera, } \\
\text { CRISTA x Neelam, FR-3 x Ex-7959, ES- } \\
1531 \text { x Heera }\end{array}$ & $\begin{array}{l}\text { EC-98994 x } \\
\text { Neelam, EC-98994 } \\
\text { x Heera, EC- } \\
104739 \text { x Ex-7959, } \\
\text { EC-115186 x Ex- } \\
7959, \text { EC-99056 x } \\
\text { Ex-7959 }\end{array}$ & $\begin{array}{l}\text { EC-112689 x } \\
\text { Neelam, ES-1474 x } \\
\text { Heera, EC-115178 x } \\
\text { Heera, EC-41752 x } \\
\text { Heera, CRISTA x } \\
\text { Neelam }\end{array}$ & - \\
\hline SG & $\begin{array}{l}\text { FR-3 x Neelam, EC- } \\
99029 \text { x Ex-7959,, } \\
\text { EC-104739 x } \\
\text { Neelam, Ex-339-6 x } \\
\text { Ex-7959, Ezox } \\
\text { Natural x Heera, } \\
\text { Ezox Natural x } \\
\text { Neelam }\end{array}$ & $\begin{array}{l}\text { Mukta x Ex-7959, Mukta x Neelam, ES-1474 } \\
\text { x Heera, ES-1496 x Heera, EC-225125 x } \\
\text { Neelam, CRISTA x Ex-7959 }\end{array}$ & $\begin{array}{l}\text { EC-104739 x } \\
\text { Neelam, Ex-5-36E } \\
\text { x Heera, EC-99029 } \\
\text { x Ex-7959, FR-3 x } \\
\text { Neelam, Ex-339-6 } \\
\text { x Ex-7959, EC- } \\
225125 \text { x Heera }\end{array}$ & $\begin{array}{l}\text { EC-112689 x Ex- } \\
7959, \text { Ex-339-6 x } \\
\text { Neelam, ES-1496 x } \\
\text { Heera, EC-115162 x } \\
\text { Neelam, EC-104739 } \\
\text { x Ex-7959 }\end{array}$ & - \\
\hline AV & $\begin{array}{l}\text { EC-41750 x Heera, } \\
\text { EC-104739 x } \\
\text { Neelam, EC-115186 } \\
\text { x Heera, ES-14600 x } \\
\text { Heera, ES-14600 x } \\
\text { Ex-7959 }\end{array}$ & $\begin{array}{l}\text { EC-112689 x Heera, Ex-28-3 x Neelam, EC- } \\
112689 \text { x Ex-7959, EC-115162 x Ex-7959, } \\
\text { EC-104739 x Ex-7959, EC-104739 x Heera }\end{array}$ & $\begin{array}{l}\text { EC-98994 x Ex- } \\
7959, \text { Ezox } \\
\text { Natural x Heera, } \\
\text { ES-1474 x Ex- } \\
7959, \text { EC-99029 x } \\
\text { Neelam, Mukta x } \\
\text { Ex-7959 }\end{array}$ & $\begin{array}{l}\text { ES-1463 x Heera, } \\
\text { Ex-28-3 x Neelam, } \\
\text { ES-1496 x Neelam, } \\
\text { EC-41752 x Ex- } \\
7959, \text { Ex-5-36E x } \\
\text { Ex-7959 }\end{array}$ & - \\
\hline $\mathbf{L N}$ & $\begin{array}{l}\text { Ex-5-36E x Ex- } \\
\text { 7959, CRISTA x } \\
\text { Heera, EC-41750 x } \\
\text { Ex-7959, CRISTA x } \\
\text { Ex-7959, Ex-5-36E } \\
\text { x Neelam }\end{array}$ & $\begin{array}{l}\text { EC-110288 x Neelam, EC-98994 x Ex-7959, } \\
\text { ES-14600 x Neelam, EC-98994 x Heera, EC- } \\
112689 \times \text { Neelam }\end{array}$ & $\begin{array}{l}\text { EC-41750 x Ex- } \\
7959, \text { EC-115186 } \\
\text { x Ex-7959, Ex-5- } \\
36 \text { E x Ex-7959, } \\
\text { EC-112689 x } \\
\text { Neelam, Ex-313- } \\
23 \text { x Heera }\end{array}$ & $\begin{array}{l}\text { T-397 x Neelam, } \\
\text { ES-14600 x } \\
\text { Neelam, CRISTA x } \\
\text { Heera, EC-112689 x } \\
\text { Neelam, ES-1496 x } \\
\text { Neelam }\end{array}$ & $\begin{array}{l}\text { EC-112689 x } \\
\text { Neelam }\end{array}$ \\
\hline LL & $\begin{array}{l}\text { FRW-9 x Neelam, } \\
\text { Ex-28-3 x Neelam, } \\
\text { Mukta x Heera, EC- } \\
104739 \text { x Heera, } \\
\text { EC-1041492 x } \\
\text { Heera }\end{array}$ & $\begin{array}{l}\text { Ex-28-3 x Ex-7959, FR-3 x Neelam, EC- } \\
1041492 \text { x Neelam, Ezox Natural x Heera, T- } \\
397 \text { x Neelam, ES-1474 x Neelam, ES-1531 x } \\
\text { Neelam }\end{array}$ & $\begin{array}{l}\text { Ex-28-3 x Neelam, } \\
\text { Ex-3 x Ex-7959, } \\
\text { Mukta x Heera, } \\
\text { EC-115178 x Ex- } \\
7959, \text { T-397 x Ex- } \\
7959\end{array}$ & $\begin{array}{l}\text { Ezox Natural x } \\
\text { Heera, Ex-28-3 x } \\
\text { Ex-7959, EC- } \\
1041492 \text { x Neelam, } \\
\text { FR-3 x Neelam, ES- } \\
1531 \text { x Neelam }\end{array}$ & - \\
\hline IV & $\begin{array}{l}\text { CRISTA x Heera, } \\
\text { Ex-5-36E x Ex- } \\
\text { 7959, CRISTA x } \\
\text { Ex-7959, Ex-5-36E } \\
\text { x Heera, FR-3 x } \\
\text { Neelam }\end{array}$ & $\begin{array}{l}\text { Ex-5-36E x Ex-7959, EC-41750 x Neelam, } \\
\text { EC-110288 x Heera, EC-112689 x Neelam, } \\
\text { ES-14600 x Neelam, EC-110288 x Neelam }\end{array}$ & $\begin{array}{l}\text { Ex-28-3 x Neelam, } \\
\text { Ezox Natural x } \\
\text { Heera, Ex-313-23 } \\
\text { x Heera, Ex-3 x } \\
\text { Ex-7959, Mukta x } \\
\text { Ex-7959 }\end{array}$ & $\begin{array}{l}\text { EC-41750 x Ex- } \\
7959, \text { Ex-3 x Heera, } \\
\text { EC-112689 x } \\
\text { Neelam, FRW-9 x } \\
\text { Neelam, ES-1463 x } \\
\text { Neelam }\end{array}$ & - \\
\hline
\end{tabular}

$\mathrm{OIL}=$ Oil $(\%), \mathrm{BD}=$ Bulk density $\left(\mathrm{g} / \mathrm{cm}^{3}\right), \mathrm{TD}=$ True density $\left(\mathrm{g} / \mathrm{cm}^{3}\right), \mathrm{PO}=$ Porosity $(\%), \mathrm{PR}=$ Protein $(\%), \mathrm{FAA}=$ Free amino acids $(\mathrm{g} / \mathrm{Kg}), \mathrm{CAR}=$ Carbohydrate $(\%), \mathrm{PHE}=\mathrm{Phenol}(\%), \mathrm{RF}=$ Refractive index, $\mathrm{SG}=$ Specific gravity, $\mathrm{AV}=\mathrm{Acid}$ value, $\mathrm{LN}=$ Linolenic acid (\%), LL= Linoleic acid (\%) and IV= Iodine value. 
Table.5 Colour and texture of seeds along with colour value of oil of 33 parents in linseed

\begin{tabular}{|c|c|c|c|c|c|}
\hline S.No. & Parents & $\begin{array}{l}\text { Colour of } \\
\text { seeds }\end{array}$ & $\begin{array}{l}\text { Colour } \\
\text { Code } \\
\text { (Kew) }\end{array}$ & Texture of seeds & Colour value of oil \\
\hline \multicolumn{6}{|c|}{ Testers } \\
\hline 1 & Heera & $\begin{array}{l}\text { Greyed } \\
\text { orange }\end{array}$ & $165 \mathrm{~A}$ & Medium \& shiny & $20+2$ \\
\hline 2 & Ex-7959 & $\begin{array}{l}\text { Greyed } \\
\text { orange }\end{array}$ & $166 \mathrm{~A}$ & Large \& shiny & $20.3+2$ \\
\hline 3 & Neelam & $\begin{array}{l}\text { Greyed } \\
\text { orange }\end{array}$ & $165 \mathrm{~A}$ & Very small \& shiny & $30+2$ \\
\hline \multicolumn{6}{|c|}{ Lines } \\
\hline 1 & EC-41750 & Greyed orange & $165 \mathrm{~A}$ & Medium \& shiny & $26.7+2$ \\
\hline 2 & EC-41752 & Greyed orange & $165 \mathrm{~A}$ & Medium \& shiny & $21.3+2$ \\
\hline 3 & EC-98994 & Greyed orange & $165 \mathrm{~A}$ & Medium \& shiny & $21.8+2$ \\
\hline 4 & T-397 & Greyed orange & $165 \mathrm{~A}$ & Medium \& shiny & $22.4+2$ \\
\hline 5 & EC-99029 & Brown & 200D & Small \& non-shiny & $22.4+2$ \\
\hline 6 & EC-99056 & Greyed orange & $166 \mathrm{~A}$ & Medium \& shiny & $20.8+2$ \\
\hline 7 & EC-104739 & Greyed orange & $166 \mathrm{~A}$ & Medium \& shiny & $30.1+2$ \\
\hline 8 & CRISTA & Greyed orange & $165 \mathrm{~A}$ & Small \& shiny & $22.4+2$ \\
\hline 9 & EC-110288 & Greyed orange & $165 \mathrm{~A}$ & Small \& shiny & $60+2$ \\
\hline 10 & EC-112689 & Brown & 200D & Medium \& non-shiny & $22.4+2$ \\
\hline 11 & EC-115161 & Greyed orange & $165 \mathrm{~A}$ & Medium \& shiny & $30+2$ \\
\hline 12 & EC-115162 & Greyed orange & $165 \mathrm{~A}$ & Small \& non-shiny & $22.2+2$ \\
\hline 13 & EC-115178 & Greyed orange & $166 \mathrm{~A}$ & Small \& non-shiny & $22.4+2$ \\
\hline 14 & EC-115186 & Greyed orange & $166 \mathrm{~A}$ & Small \& non-shiny & $22.3+2$ \\
\hline 15 & EC-225125 & Greyed orange & $165 \mathrm{~A}$ & Medium \& non-shiny & $21.2+2$ \\
\hline 16 & EC-1041492 & Greyed orange & $166 \mathrm{~A}$ & Small \& shiny & $22.3+2$ \\
\hline 17 & Mukta & Greyed orange & $165 \mathrm{~A}$ & Medium \& shiny & $22.3+2$ \\
\hline 18 & ES-1463 & Greyed orange & $165 \mathrm{~B}$ & Medium \& shiny & $22.8+2$ \\
\hline 19 & ES-1474 & Greyed orange & $165 \mathrm{~B}$ & Small \& non-shiny & $61+2$ \\
\hline 20 & ES-1496 & Greyed orange & $165 \mathrm{~A}$ & Small \& shiny & $35+2.1$ \\
\hline 21 & ES-1531 & Greyed orange & $165 \mathrm{~A}$ & Small \& shiny & $45.3+3$ \\
\hline 22 & ES-14600 & Greyed orange & $165 \mathrm{~A}$ & Small \& shiny & $70+2$ \\
\hline 23 & Ex-3 & Greyed orange & $165 \mathrm{~A}$ & Medium \& non-shiny & $30+2$ \\
\hline 24 & $E x-5-36 E$ & Greyed orange & $165 \mathrm{~A}$ & Medium \& non-shiny & $11.9+2$ \\
\hline 25 & Ex-28-3 & Greyed orange & $165 \mathrm{~A}$ & Small \& non-shiny & $22.3+2$ \\
\hline 26 & Ex-313-23 & Greyed orange & $165 \mathrm{~A}$ & Small \& non-shiny & $60+2$ \\
\hline 27 & Ex-339-6 & Greyed orange & $165 \mathrm{~A}$ & Medium \& non-shiny & $22.5+2$ \\
\hline 28 & Ezox Natural & Greyed orange & $165 \mathrm{~A}$ & Medium \& non-shiny & $61.7+3$ \\
\hline 29 & FR-3 & Brown & 200D & Small \& non-shiny & $22.1+2$ \\
\hline 30 & FRW-9 & Greyed orange & $177 \mathrm{~A}$ & Medium \& non-shiny & $35+2.1$ \\
\hline
\end{tabular}


Table.6 Colour and texture of seeds along with colour value of oil of 90 crosses in F1 generation in linseed

\begin{tabular}{|c|c|c|c|c|c|}
\hline S.No. & Crosses & $\begin{array}{l}\text { Colour of } \\
\text { seeds }\end{array}$ & $\begin{array}{c}\text { Colour } \\
\text { code } \\
\text { (Kew) }\end{array}$ & Texture of seeds & $\begin{array}{c}\text { Colour } \\
\text { value of oil }\end{array}$ \\
\hline 1 & EC-41750 x Heera & Brown & 200D & Large \& non shiny & $19+2$ \\
\hline 2 & EC-41750 x Ex-7959 & Brown & 200D & Large \& non shiny & $48.5+2$ \\
\hline 3 & EC-41750 x Neelam & Brown & 200D & Medium \& non-shiny & $24+2$ \\
\hline 4 & EC-41752 x Heera & Greyed orange & $165 \mathrm{~A}$ & Medium \& non-shiny & $10+0.3$ \\
\hline 5 & EC-41752 x Ex-7959 & Greyed orange & $165 \mathrm{~A}$ & Medium \& shiny & $31+2$ \\
\hline 6 & EC-41752 x Neelam & Greyed orange & $165 \mathrm{~A}$ & Medium \& shiny & $27+2$ \\
\hline 7 & EC-98994 x Heera & Greyed orange & $165 \mathrm{~A}$ & Medium \& non-shiny & $30.9+2$ \\
\hline 8 & EC-98994 x Ex-7959 & Greyed orange & $165 \mathrm{~A}$ & Large \& shiny & $45.5+2$ \\
\hline 9 & EC-98994 x Neelam & Greyed orange & $165 \mathrm{~A}$ & Large \& shiny & $31.5+2$ \\
\hline 10 & T-397 x Heera & Greyed orange & $165 \mathrm{~A}$ & Medium \& & +2 \\
\hline 11 & T-397 x Ex-7959 & Greyed & & Large \& & \\
\hline 12 & T-397 x Neelam & Greyed orange & $165 \mathrm{~A}$ & Large \& & $40+2$ \\
\hline 13 & EC-99029 x Heera & Greyed orange & $165 \mathrm{~A}$ & Medium \& non-shiny & $26+2$ \\
\hline 14 & EC-99029 x Ex-7959 & Greyed orange & $165 \mathrm{~A}$ & Large \& non shiny & $43.5+2$ \\
\hline 15 & EC-99029 x Neelam & Brown & 200D & Large \& shiny & $21+2$ \\
\hline 16 & EC-99056 x Heera & Greyed orange & $165 \mathrm{~A}$ & Large \& shiny & $0+2$ \\
\hline 17 & EC-99056 x Ex-7959 & Greyed orange & $165 \mathrm{~A}$ & Small \& non-shiny & $30+2$ \\
\hline 18 & EC-99056 x Neelam & Greyed orange & $165 \mathrm{~A}$ & Medium \& s & $50.5+2$ \\
\hline 19 & EC-104739 x Heera & & & & \\
\hline 20 & EC-1047 & Gre & & & \\
\hline 21 & EC-104739 x Neelam & Greyed o & $165 \mathrm{~A}$ & Small \& no & $30+2$ \\
\hline 22 & CRISTA $x$ Heera & Greyed orange & $166 \mathrm{~A}$ & Medium \& non-shiny & $35+2$ \\
\hline 23 & CRISTA x Ex-7959 & Greyed orange & $165 \mathrm{~A}$ & Medium \& shiny & $38.5+2$ \\
\hline 24 & CRISTA x Neelam & Greyed orange & $166 \mathrm{~A}$ & Medium \& shiny & $27.5+$ \\
\hline 25 & EC-110288 x Heera & Brown & 200D & Large \& non shiny & $36+2$ \\
\hline 26 & EC-110288 x Ex-7959 & Greyed orange & $165 \mathrm{~A}$ & Medium \& non-shiny & $35+2$ \\
\hline 27 & EC-110288 x Neelam & Greyed orange & $165 \mathrm{~A}$ & Medium \& shiny & $25+2$ \\
\hline 28 & EC-112689 x Heera & Gre & $166 \mathrm{~A}$ & \& shiny & $32.5+2$ \\
\hline 29 & EC-112689 x Ex-7959 & Greyed orange & $165 \mathrm{~A}$ & Large \& non shiny & $38+2$ \\
\hline 30 & EC-112689 x Neelam & Greyed orange & $165 \mathrm{~A}$ & Medium \& shiny & $47.5+2$ \\
\hline 31 & EC-115161 x Heera & Greyed orange & $166 \mathrm{~A}$ & Medium \& shiny & $45+2$ \\
\hline 32 & EC-115161 x Ex-7959 & Greyed orange & $165 \mathrm{~A}$ & Large \& shiny & $30+2$ \\
\hline 33 & EC-115161 x Neelam & & & & \\
\hline 34 & & & & & \\
\hline 35 & EC-115162 x Ex-7959 & Greyed orange & & Large \& shiny & $31+2$ \\
\hline 36 & EC-115162 x Neelam & Greyed orange & $165 \mathrm{~A}$ & Large \& shiny & $30+2$ \\
\hline 37 & EC-115178 x Heera & Greyed orange & $165 \mathrm{~A}$ & Large \& shiny & $25+2$ \\
\hline 38 & EC-115178 x Ex-7959 & Greyed orange & $165 \mathrm{~A}$ & Large \& shiny & $30+2$ \\
\hline 39 & EC-115178 x Neelam & Brown & 200D & Small \& non-shiny & $27.5+2$ \\
\hline 40 & EC-115186 x Heera & Greyed orange & $166 \mathrm{~A}$ & Small \& non-shiny & $28+2$ \\
\hline 41 & EC-115186 x Ex-7959 & Greyed or: & & Im \& shiny & \\
\hline 42 & EC-115186 x Neelam & $\mathrm{d}$ ora & & m \& shiny & $39.5+2$ \\
\hline 43 & EC-225125 x Heera & Greyed orange & $165 \mathrm{~A}$ & Medium \& shiny & $31+2$ \\
\hline 44 & EC-225125 x Ex-7959 & Greyed orange & $165 \mathrm{~A}$ & Medium \& shiny & $29.5+2$ \\
\hline 45 & EC-225125 x Neelam & Greyed orange & $165 \mathrm{~A}$ & Large \& shiny & $33+2$ \\
\hline
\end{tabular}


Continued..........

\begin{tabular}{|c|c|c|c|c|c|}
\hline S.No & Crosses & $\begin{array}{l}\text { Colour of } \\
\text { seeds }\end{array}$ & $\begin{array}{l}\text { Colour } \\
\text { Code } \\
\text { (Kew) }\end{array}$ & Texture of seeds & $\begin{array}{l}\text { Colour } \\
\text { value }\end{array}$ \\
\hline 46 & EC-1041492 x Heera & Greyed orange & $165 \mathrm{~A}$ & Medium \& shiny & $29+1$ \\
\hline 47 & EC-1041492 x Ex-7959 & Greyed orange & $166 \mathrm{~A}$ & Medium \& non-shiny & $40+2$ \\
\hline 48 & EC-1041492 x Neelam & Greyed orange & $166 \mathrm{~A}$ & Medium \& shiny & $20.5+0.4$ \\
\hline 49 & Mukta x Heera & Brown & 200D & Large \& shiny & $47.5+2$ \\
\hline 50 & Mukta x Ex-7959 & Greyed orange & $165 \mathrm{~A}$ & Large \& shiny & $20+2$ \\
\hline 51 & Mukta x Neelam & Greyed orange & $165 \mathrm{~A}$ & Large \& shiny & $29+2$ \\
\hline 52 & ES-1463 x Heera & Greyed orange & $165 \mathrm{~A}$ & Medium \& shiny & $32.5+2$ \\
\hline 53 & ES-1463 x Ex-7959 & Greyed orange & $165 \mathrm{~A}$ & Small \& shiny & $48+2$ \\
\hline 54 & ES-1463 x Neelam & Greyed orange & $166 \mathrm{~A}$ & Medium \& shiny & $18+2$ \\
\hline 55 & ES-1474 x Heera & Greyed orange & $165 \mathrm{~A}$ & Large \& shiny & $30+1$ \\
\hline 56 & ES-1474 x Ex-7959 & Greyed orange & $165 \mathrm{~A}$ & Large \& shiny & $50+2$ \\
\hline 57 & ES-1474 x Neelam & Greyed orange & $165 \mathrm{~A}$ & Medium \& shiny & $35+2$ \\
\hline 58 & ES-1496 x Heera & Greyed orange & $165 \mathrm{~A}$ & Medium \& shiny & $29+1$ \\
\hline 59 & ES-1496 x Ex-7959 & Greyed orange & $165 \mathrm{~A}$ & Large \& shiny & $28+2$ \\
\hline 60 & ES-1496 x Neelam & Greyed orange & $165 \mathrm{~A}$ & Medium \& shiny & $44+2$ \\
\hline 61 & ES-1531 x Heera & Greyed orange & $165 \mathrm{~A}$ & Medium \& shiny & $46.5+2$ \\
\hline 62 & ES-1531 x Ex-7959 & Greyed orange & $165 \mathrm{~A}$ & Large \& shiny & $34.5+2$ \\
\hline 63 & ES-1531 x Neelam & Brown & 200D & Medium \& shiny & $21+2$ \\
\hline 64 & ES-14600 x Heera & Brown & $200 \mathrm{D}$ & Medium \& shiny & $46+2$ \\
\hline 65 & ES-14600 x Ex-7959 & Brown & 200D & Medium \& shiny & $45+2$ \\
\hline 66 & ES-14600 x Neelam & Greyed orange & $165 \mathrm{~A}$ & Medium \& non-shiny & $18.5+2$ \\
\hline 67 & Ex-3 x Heera & Brown & 200D & Small \& non-shiny & $31+2$ \\
\hline 68 & Ex-3 x Ex-7959 & Greyed orange & $165 \mathrm{~A}$ & Large \& non shiny & $48.5+2$ \\
\hline 69 & Ex-3 x Neelam & Greyed orange & $166 \mathrm{~A}$ & Large \& shiny & $25.8+2$ \\
\hline 70 & Ex-5-36E x Heera & Greyed orange & $165 \mathrm{~A}$ & Large \& shiny & $35+2$ \\
\hline 71 & Ex-5-36E x Ex-7959 & Greyed orange & $165 \mathrm{~A}$ & Large \& shiny & $56+2$ \\
\hline 72 & Ex-5-36E x Neelam & Greyed orange & $165 \mathrm{~A}$ & Medium \& shiny & $45+2$ \\
\hline 73 & Ex-28-3 x Heera & Brown & 200D & Medium \& shiny & $33.5+2$ \\
\hline 74 & Ex-28-3 x Ex-7959 & Greyed orange & $165 \mathrm{~A}$ & Medium \& shiny & $50.5+2$ \\
\hline 75 & Ex-28-3 x Neelam & Greyed orange & $165 \mathrm{~A}$ & Medium \& shiny & $31+2$ \\
\hline 76 & Ex-313-23 x Heera & Greyed orange & $165 \mathrm{~A}$ & Large \& shiny & $20.8+2$ \\
\hline 77 & Ex-313-23 x Ex-7959 & Greyed orange & $165 \mathrm{~A}$ & Medium \& shiny & $38+2$ \\
\hline 78 & Ex-313-23 x Neelam & Greyed orange & $165 \mathrm{~A}$ & Medium \& shiny & $16.5+0.5$ \\
\hline 79 & Ex-339-6 x Heera & Greyed orange & $166 \mathrm{~A}$ & Medium \& non-shiny & $29.5+2$ \\
\hline 80 & Ex-339-6 x Ex-7959 & Greyed orange & $165 \mathrm{~A}$ & Large \& shiny & $40+2$ \\
\hline 81 & Ex-339-6 x Neelam & Brown & 200D & Medium \& non-shiny & $31+2$ \\
\hline 82 & Ezox Natural x Heera & Greyed orange & $165 \mathrm{~A}$ & Medium \& shiny & $30+2$ \\
\hline 83 & Ezox Natural x Ex-7959 & Brown & 200D & Large \& shiny & $44+2$ \\
\hline 84 & Ezox Natural x Neelam & Greyed orange & $165 \mathrm{~A}$ & Medium \& shiny & $47+2$ \\
\hline 85 & FR-3 x Heera & Greyed orange & $165 \mathrm{~A}$ & Medium \& shiny & $56+2$ \\
\hline 86 & FR-3 x Ex-7959 & Greyed orange & $165 \mathrm{~A}$ & Medium \& shiny & $37.8+2$ \\
\hline 87 & FR-3 x Neelam & Greyed orange & $165 \mathrm{~A}$ & Small \& non-shiny & $47.5+2$ \\
\hline 88 & FRW-9 x Heera & Greyed orange & $165 \mathrm{~A}$ & Medium \& shiny & $38+2$ \\
\hline 89 & FRW-9 x Ex-7959 & Greyed orange & $165 \mathrm{~A}$ & Large \& shiny & $40+2$ \\
\hline 90 & FRW-9 x Neelam & Brown & 200D & Medium \& shiny & $47+2$ \\
\hline
\end{tabular}


Table.7 Colour and texture of seeds along with colour value of oil of 90 crosses in F2 generation in linseed

\begin{tabular}{|c|c|c|c|c|c|}
\hline S.No. & Crosses & $\begin{array}{l}\text { Colour of } \\
\text { seeds }\end{array}$ & $\begin{array}{c}\text { Colour } \\
\text { Code } \\
\text { (Kew) }\end{array}$ & Texture of seeds & $\begin{array}{c}\text { Colour } \\
\text { value }\end{array}$ \\
\hline 1 & EC-41750 x Heera & Brown & 200D & Small \& non-shiny & $40+2$ \\
\hline 2 & EC-41750 x Ex-7959 & Brown & 200D & Small \& non-shiny & $30+2$ \\
\hline 3 & EC-41750 x Neelam & Brown & 200D & Medium \& shiny & $40+2$ \\
\hline 4 & EC-41752 x Heera & Brown & 200D & Large \& non shiny & $50+2$ \\
\hline 5 & EC-41752 x Ex-7959 & Brown & 200D & Large \& non shiny & $50+2$ \\
\hline 6 & EC-41752 x Neelam & Brown & 200D & Medium \& shiny & $60+2$ \\
\hline 7 & EC-98994 x Heera & Brown & 200D & Medium \& shiny & $50+2$ \\
\hline 8 & EC-98994 x Ex-7959 & Brown & 200D & Medium \& non-shiny & $35+2$ \\
\hline 9 & EC-98994 x Neelam & Brown & 200D & Large \& non shiny & $15+1$ \\
\hline 10 & T-397 x Heera & Brown & 200D & Medium \& shiny & $60+2$ \\
\hline 11 & T-397 x Ex-7959 & Brown & 200D & Medium \& shiny & $60+2$ \\
\hline 12 & T-397 x Neelam & Brown & 200D & Medium \& shiny & $40+2$ \\
\hline 13 & EC-99029 x Heera & Brown & 200D & Medium \& shiny & $85+2$ \\
\hline 14 & EC-99029 x Ex-7959 & Brown & 200D & Medium \& shiny & $40+2$ \\
\hline 15 & EC-99029 x Neelam & Brown & 200D & Medium \& non-shiny & $40+2$ \\
\hline 16 & EC-99056 x Heera & Greyed orange & $165 \mathrm{~A}$ & Large \& non shiny & $25+2$ \\
\hline 17 & EC-99056 x Ex-7959 & Brown & $200 \mathrm{D}$ & Large \& non shiny & $30+2$ \\
\hline 18 & EC-99056 x Neelam & Brown & 200D & Medium \& shiny & $30+2$ \\
\hline 19 & EC-104739 x Heera & Brown & 200D & Large \& non shiny & $30+2$ \\
\hline 20 & EC-104739 x Ex-7959 & Brown & 200D & Large \& shiny & $30+2$ \\
\hline 21 & EC-104739 x Neelam & Greyed orange & $165 \mathrm{~A}$ & Large \& non shiny & $15+1$ \\
\hline 22 & CRISTA $x$ Heera & Brown & 200D & Small \& non-shiny & $40+2$ \\
\hline 23 & CRISTA x Ex-7959 & Brown & 200D & Medium \& non-shiny & $30+2$ \\
\hline 24 & CRISTA x Neelam & Brown & 200D & Medium \& shiny & $15+2$ \\
\hline 25 & EC-110288 x Heera & Brown & 200D & Large \& non shiny & $40+2$ \\
\hline 26 & EC-110288 x Ex-7959 & Brown & 200D & Small \& non-shiny & $30+2$ \\
\hline 27 & EC-110288 x Neelam & Brown & $200 \mathrm{D}$ & Small \& non-shiny & $40+2$ \\
\hline 28 & EC-112689 x Heera & Brown & 200D & Large \& shiny & $50+2$ \\
\hline 29 & EC-112689 x Ex-7959 & Brown & 200D & Medium \& shiny & $30+2$ \\
\hline 30 & EC-112689 x Neelam & Brown & 200D & Medium \& shiny & $50+2$ \\
\hline 31 & EC-115161 x Heera & Brown & $200 \mathrm{D}$ & Medium \& shiny & $25+1$ \\
\hline 32 & EC-115161 x Ex-7959 & Brown & 200D & Large \& shiny & $65+2$ \\
\hline 33 & EC-115161 x Neelam & Brown & 200D & Large \& shiny & $30+2$ \\
\hline 34 & EC-115162 x Heera & Brown & 200D & Medium \& shiny & $30+2$ \\
\hline 35 & EC-115162 x Ex-7959 & Brown & 200D & Medium \& non-shiny & $50+2$ \\
\hline 36 & EC-115162 x Neelam & Brown & 200D & Large \& shiny & $55+2$ \\
\hline 37 & EC-115178 x Heera & Brown & 200D & Large \& shiny & $30+1$ \\
\hline 38 & EC-115178 x Ex-7959 & Brown & 200D & Large \& shiny & $25+1$ \\
\hline 39 & EC-115178 x Neelam & Brown & $200 \mathrm{D}$ & Small \& non-shiny & $20+2$ \\
\hline 40 & EC-115186 x Heera & Brown & 200D & Small \& non-shiny & $30+2$ \\
\hline 41 & EC-115186 x Ex-7959 & Brown & 200D & Medium \& shiny & $15+1$ \\
\hline 42 & EC-115186 x Neelam & Brown & $200 \mathrm{D}$ & Medium \& shiny & $30+2$ \\
\hline 43 & EC-225125 x Heera & Brown & $200 \mathrm{D}$ & Medium \& shiny & $40+2$ \\
\hline 44 & EC-225125 x Ex-7959 & Brown & 200D & Small \& non-shiny & $50+2$ \\
\hline 45 & EC-225125 x Neelam & Brown & 200D & Large \& shiny & $15+1$ \\
\hline
\end{tabular}


Continued..........

\begin{tabular}{|c|c|c|c|c|c|}
\hline S.No & Crosses & $\begin{array}{l}\text { Colour of } \\
\text { seeds }\end{array}$ & $\begin{array}{c}\text { Colour } \\
\text { Code } \\
\text { (Kew) }\end{array}$ & Texture of seeds & $\begin{array}{c}\text { Colour } \\
\text { value }\end{array}$ \\
\hline 46 & EC-1041492 x Heera & Brown & $200 \mathrm{D}$ & Medium \& shiny & $25+1$ \\
\hline 47 & EC-1041492 x Ex-7959 & Brown & 200D & Medium \& non-shiny & $15+1$ \\
\hline 48 & EC-1041492 x Neelam & Brown & 200D & Medium \& shiny & $30+2$ \\
\hline 49 & Mukta x Heera & Brown & 200D & Large \& shiny & $30+2$ \\
\hline 50 & Mukta x Ex-7959 & Brown & 200D & Large \& shiny & $30+2$ \\
\hline 51 & Mukta x Neelam & Brown & 200D & Large \& shiny & $30+2$ \\
\hline 52 & ES-1463 x Heera & Brown & 200D & Medium \& shiny & $40+2$ \\
\hline 53 & ES-1463 x Ex-7959 & Brown & 200D & Medium \& non-shiny & $30+2$ \\
\hline 54 & ES-1463 x Neelam & Brown & 200D & Medium \& shiny & $30+2$ \\
\hline 55 & ES-1474 x Heera & Brown & 200D & Large \& shiny & $15+1$ \\
\hline 56 & ES-1474 x Ex-7959 & Brown & 200D & Medium \& shiny & $70+2$ \\
\hline 57 & ES-1474 x Neelam & Brown & 200D & Medium \& shiny & $50+2$ \\
\hline 58 & ES-1496 x Heera & Brown & 200D & Medium \& shiny & $30+2$ \\
\hline 59 & ES-1496 x Ex-7959 & Brown & 200D & Large \& shiny & $40+2$ \\
\hline 60 & ES-1496 x Neelam & Brown & $200 \mathrm{D}$ & Medium \& shiny & $40+2$ \\
\hline 61 & ES-1531 x Heera & Brown & 200D & Medium \& shiny & $15+1$ \\
\hline 62 & ES-1531 x Ex-7959 & Brown & 200D & Large \& shiny & $25+1$ \\
\hline 63 & ES-1531 x Neelam & Brown & 200D & Medium \& shiny & $15+1$ \\
\hline 64 & ES-14600 x Heera & Brown & 200D & Medium \& shiny & $40+2$ \\
\hline 65 & ES-14600 x Ex-7959 & Brown & 200D & Medium \& shiny & $30+2$ \\
\hline 66 & ES-14600 x Neelam & Brown & 200D & Medium \& non-shiny & $55+2$ \\
\hline 67 & Ex-3 x Heera & Brown & 200D & Small \& non-shiny & $50+2$ \\
\hline 68 & Ex-3 x Ex-7959 & Brown & 200D & Large \& shiny & $25+2$ \\
\hline 69 & Ex-3 x Neelam & Brown & $200 \mathrm{D}$ & Large \& shiny & $15+1$ \\
\hline 70 & Ex-5-36E x Heera & Brown & 200D & Large \& shiny & $16+1$ \\
\hline 71 & Ex-5-36E x Ex-7959 & Brown & 200D & Large \& shiny & $15+1$ \\
\hline 72 & Ex-5-36E x Neelam & Brown & 200D & Medium \& shiny & $15+1$ \\
\hline 73 & Ex-28-3 x Heera & Brown & $200 \mathrm{D}$ & Medium \& non-shiny & $15+1$ \\
\hline 74 & Ex-28-3 x Ex-7959 & Brown & 200D & Medium \& shiny & $50+2$ \\
\hline 75 & Ex-28-3 x Neelam & Brown & 200D & Medium \& shiny & $15+1$ \\
\hline 76 & Ex-313-23 x Heera & Brown & 200D & Large \& shiny & $50+2$ \\
\hline 77 & Ex-313-23 x Ex-7959 & Brown & 200D & Medium \& shiny & $50+2$ \\
\hline 78 & Ex-313-23 x Neelam & Brown & $200 \mathrm{D}$ & Medium \& non-shiny & $60+2$ \\
\hline 79 & Ex-339-6 x Heera & Brown & 200D & Medium \& non-shiny & $30+2$ \\
\hline 80 & Ex-339-6 x Ex-7959 & Brown & 200D & Large \& non shiny & $50+2$ \\
\hline 81 & Ex-339-6 x Neelam & Greyed orange & $165 \mathrm{~A}$ & Medium \& non-shiny & $40+2$ \\
\hline 82 & Ezox Natural x Heera & Brown & 200D & Medium \& non-shiny & $40+2$ \\
\hline 83 & Ezox Natural x Ex-7959 & Brown & 200D & Large \& non shiny & $30+2$ \\
\hline 84 & Ezox Natural x Neelam & Brown & 200D & Medium \& shiny & $30+2$ \\
\hline 85 & FR-3 x Heera & Brown & 200D & Medium \& shiny & $70+2$ \\
\hline 86 & FR-3 x Ex-7959 & Brown & $200 \mathrm{D}$ & Medium \& shiny & $15+1$ \\
\hline 87 & FR-3 x Neelam & Brown & 200D & Small \& non-shiny & $30+2$ \\
\hline 88 & FRW-9 x Heera & Brown & 200D & Medium \& shiny & $65+2$ \\
\hline 89 & FRW-9 x Ex-7959 & Brown & 200D & Medium \& shiny & $15+1$ \\
\hline 90 & FRW-9 x Neelam & Brown & 200D & Medium \& shiny & $15+1$ \\
\hline
\end{tabular}


High SCA effects manifested by low $\times$ low crosses may be attributed to dominance $\times$ dominance type of non-allelic gene interaction producing over dominance thus being non-fixable. If both the GCA and SCA values are not significant, epistatic gene effects may play a remarkable role in determining these characters (Talukder et al., 2016).

Considering the GCA effects of parents, none of the parent was found to be good general combiner for all the traits to utilize them for edible purposes. Among the testers based on the per se performance and gca effect in both generations, Heera was found good general combiner for true density; tester parent Ex7959 for porosity and phenol content and Neelam for odine value, linolenic acid and linoleic acid. Among lines based on per se performance and gca effect in both generations Ex-5-36E was good general combiner for iodine value. While considering the per se performance of parents alone, EC1041492 was good general combiner for true density, phenol, acid value, iodine value and linolenic acid; Ex-28-3 for porosity, free amino acids, specific gravity and acid value; Ex-339-6 for true density, oil percentage and refractive index; Ex-313-23 for bulk density, true density, refractive index and linolenic acid; Mukta for free amino acids, refractive index, iodine value and linolenic acid and Ezox Natural for specific gravity and acid value. On the basis of gca effect of parent over both generations, parent Ex-5-36E was good general combiner phenol, iodine value and linolenic acid; Ex-313-23 for porosity; EC-112689 for linoleic acid; EC-104739 for acid value and linoleic acid and Ex-28-3 for bulk density and iodine value. Parent EC112689 was found good general combiner for oil content in both generations. Similarly, parents EC-115161 and EC-115178 were good general combiner for oil in $\mathrm{F}_{1}$ generation while CRISTA for in $\mathrm{F}_{2}$ generation. It is therefore suggested that intermating in randomly selected progenies from early segregating generations (biparental mating) obtained as a result of crossing between parents will release the concealed genetic variability through breakage of undesirable linkages involved in various traits, may produce population for selection of elite lines in advance generations (Bhateria et al., 2006).

The specific combining ability is defined as any deviation in the performance of parents in producing good cross combinations (Hallauer and Miranda, 1981 and Yamanura et al., 2009). Falconer (1981) suggested that high SCA variances showed non-additive gene action or dominance and epistatic gene effects. A perusal of results indicated that none of the cross combinations was superior for all the traits.In the present study good cross combinations was obtained not only between high $\mathrm{x}$ high general combiners but also as a result of low $\mathrm{x}$ low and low $\mathrm{x}$ high combinations interaction. The good combinations from high $\mathrm{x}$ low general combiners might be due to the favourable additive effects of good general combiner parent and epistatic effect of poor general combiner and low $\mathrm{x}$ low general combiners might be due to the presence of epistatic or dominant gene actions producing over dominance (Bhateria et al., 2006).Cross combination EC-112689 x Neelam was found good specific combiner for oil based on per se performance in $\mathrm{F}_{1}$ generation.. However, cross Ex-3 x Ex-7959 forporosity, oil percentage, iodine value and linoleic acid; cross EC-115186 x Ex for refractive index and linolenic acid, and cross Ezox Natural $\mathrm{x}$ Heera for protein, acid value and iodine value were good specific combiner in $\mathrm{F}_{1}$ generation. Crosses EC-98994 x Ex, EC-110288 x Neelam, ES-1463 x Neelam and Mukta x Ex7959 were good specific combiners in $F_{1}$ generation. Similarly, cross EC-41750 x Ex7959 for phenol and iodine value; EC-41752 
x Ex-7959 also for porosity, free amino acids, oil percentage and acid value and cross EC1041492 x Neelam for free amino acids and linoleic acid in $F_{2}$ generation were good specific combiners. Beside this, cross EC115178 x Heera was good specific combiner for true density in both generation, bulk density and porosity in $\mathrm{F}_{1}$ generation and refractive index in $\mathrm{F}_{2}$ generation, cross EC99056 x Ex-7959 for free amino acids and phenol in both generations in $F_{1}$ generation and for protein in $\mathrm{F}_{2}$ generation, cross FR-3 x Heera for test weight and carbohydrate content in $\mathrm{F}_{2}$ generation, cross EC-112689 x Neelam for linolenic acid in both generations and for refractive index and iodine value in $\mathrm{F}_{2}$ generation and cross ES-1463 x Neelam for bulk density, true density and oil percentage in $F_{1}$ generation and free amino acids and iodine value in $F_{2}$ generation were good specific combiners. The cross FRW-9 x Heera for carbohydrate and cross EC-110288 x Heera for phenol were good specific combiner in both generations. Finally the crosses EC-104739 x Neelam, EC-110288 x Neelam, EC-112689 x Neelam, EC-115178 x Heera and Ex-339-6 x Heera in $F_{1}$ and crosses EC-41750 x Heera, EC-41752 x Ex-7959, EC-1041492 x Neelam, ES-1474 x Heera and Ezox Heera in $\mathrm{F}_{2}$ generation and cross Ex-283 x Ex-7959 in both generations were good specific combiners for oil percentage and improvement of these crosses can be done using biparental mating or reciprocal recurrent selection followed by pedigree selection (Bhateria et al., 2006 and Ilker et al., 2000).

\section{References}

Aslan, M., Didem, D. O., Nilufer, O., Ekrem, S. and Erdem, Y. (2007). In vivo antidiabetic and antioxidant potential of Helichrysum plicatums sp. plicatum capitulums in streptozotocin-induced- diabetic rats. Journal of Ethnopharmacology, 109: 54-59.

Basbag, S., Toncer, O. and Basbag, M. (2009). Fatty acid composition of Linum spp. collected from southeastern of Turkey. Chemistry of Natural Compdounds, 45(3): 411-413.

Bayrak, A., Kiralan, M., Ipek, A., Arslan, N., Cosge, B. and Khawar, K.M. 2010. Fatty acid compositions of linseed (Linum usitatissimum L.) genotypes of different origin cultivated in Turkey. Biotechnology and Biotechnological Equipments, 24(2): 1836-1842.

Bhateria, S., Sood, S.P. and Pathania, A. (2006). Genetic analysis of quantitative traits across environments in linseed (Linum usitatissimum L.). Euphytica,150(1-2): 185-194.

Biradar, S. A., Ajithkumar, K., Rajanna, B., Savitha A. S., Shubha, G. V., Shankergoud, I., Chittapur, B. M. and Singh, P.K. (2016). Prospects and challenges in linseed (Linum usitatissimum L.) production: A review.Journal of Oilseeds Research, 33(1): 1-13.

Biswas, T.K., Sana N.K., Badal R.K. and Huque E. M. (2001). Biochemical study of some oilseeds (Brassica, Seasame and Linseed). Pakistan Journal of Biological Sciences,4(8): 1002-1005.

Bozan, B. and Temelli, F. (2008). Chemical composition and oxidative stability of flax, safflower and poppy seed and seed oils. Bioresource Technology, 99(14): 6354-6359.

Chauhan, M. P., Singh, S. and Singh, A. K. (2009). Post harvest uses of Linseed. Journal of Human Ecology,28(3): 217-219.

Chen, J., Wang, L. and Thompson, L.U. (2006). Flaxseed and its components reduce metastatis after surgical excision of solid human breast tumor 
in nude mice. Cancer Letters, 234(2): 168-75.

Coskuner, Y. and Karababa, E. (2007). Some physical properties of flaxseed (Linum usitatissimum L.). Journal of. Food Engineering, 78: 1067-1073.

Dash, J., Naik, B. S. and Mohapatra, U. B. (2017). Linseed: A valuable crop plant. International Journal of Advance Research, 5(3): 1428-1442.

Devi, K. R., Venkanna, V., Satish Chandra, B. and Hari, Y. (2018). Gene action and combining ability for yield and quality traits in rice (Oryza sativa L.) using diallel analysis. International Journal of Current Microbiology and Applied Sciences, 7(1):2319-7706.

Ebrahimi, M., Rajion, M. A. and Goh, Y. M. (2014). Effects of Oils Rich in Linoleic and $\alpha$-Linolenic Acids on Fatty Acid Profile and Gene Expression in Goat Meat. Nutrients, 6(9): 3913-3928.

Faintuch, J., Bortolotto, L. A., Marques, P. C., Faintuch, J. J., Franca, J.I. and Cecconello, I.(2011). Systemic inflammation and carotid diameter in obese patients: pilot comparative study w with flaxseed powder and cassava powder. Nutrición Hospitalaria, 26:208-213.

Falconer, D.S. (1981). Introduction to quantitative genetics, $2^{\text {nd }}$ Edition. London, New York, Longmans, pp. 340.

Fasahat, P. A., Rajabi, A., Rad, J. M. and Derera, J. (2016). Principles and Utilization of Combining Ability in Plant Breeding. Biometrics and Biostatistics International Journal, 4(1): 00085.

Fellows, P., Franco, E. and Rios, W. 1998. Starting a Small Food Processing Business. IT Publications. pp. 35.

Ganorkar, P. M. and Jain, R. K. (2013). Flaxseed - a nutritional punch.
International Food Research Journal, 20(2): 519-525.

Goyal, A., Sharma, V., Upadhyay, N., Gill, S. and Sihag, M.(2014). Flax and flaxseed oil: an ancient medicine and modern functional food. Journal of Food Science and Technology, 51(9):1633-1653.

Guan, Y., Jia, C. and Xia, Z. (1998). Mixed functional oil. International Patent, CN1176058.

Hall, Linda M., Booker, H., Siloto,R. M.P., Jhala A. J. and Weselake R. J. (2016). Flax (Linum usitatissimum L.). Industrial Oil Crops, First Edition, pp. 157-194.

Hallauer, A.R. and Fo Miranda, J.B. (1981). Quantitative genetics in maize breeding. Ames: Iowa State University Press, pp. 468.

Hamilton, P.B. and Van Slyke, D. D. (1943). Amino acids determination with ninhydrin. J. Biological Chemistry, 150: 231-233.

Herchi, W., Harrabi, S., Sebei, K., Rochut, S., Boukhchina, S., Pepe, C. and Kallel H. (2009). Phytosterol accumulation in the seeds of Linum usitatissimum L. Plant Physiology and Biochemistry, 47: 880-885.

Hongzhi, Y., Zhihuai, M. and Hequn, T. (2004). Determination and removal methods of cyanogenic glucoside in flaxseed. ASAE/CSAE meeting presentation: 04066.

Hussain, S., Anjum, F.M., Butt, M.S. and Sheikh, M.A. (2008). Chemical compositions and functional properties of Flaxseed flour. Sarhad Journal of Agriculture, 24(4): 649-653.

Ilker, E., Tonk, F.A., Tosun, M., Altinbas, M. and Kuçukakça, M. (2009). Inheritance and combining ability in some powdery mildew resistant wheat lines. Crop Breeding and Applied Biotechnology, 9: 124-131. 
Indrayan, A.K., Kumar, N., Tyagi, P.K. and Sharma, V. (2005). Chemical investigation of the seed oil of Strychnos potatorum and spectroscopic estimation of Linoleic and Linolenic acids. Indian Journal of Chemistry, 44B: 1324-1326.

Jaswir, I., Krrrs, D.D., Man, Y.B.C. and Hassan, T.H. (2005). Physio-chemical stability of flaxseed oil with natural antioxidant mixtures during heating. Journal of Oleo Science, 54(2): 71-79.

Kaur, P., Waghmare, R., Kumar, V., Rasane, P., Kaur, S. and Gat, Y. (2018). Recent advances in utilization of flaxseed as potential source for value addition. Oilseeds and Fats, Crops and Lipids, 25(3): A304.

Khan, M.L., Sharif, M., Sarwar, M., Sameea and Ameen, M. (2010). Chemical composition of different varieties of Linseed. Pakistan Veterinary Journal, 30(2): 79-82.

Kouba, M., Enser, M., Whittington, F.M., Nute, G.R. and Wood, J.D. (2003). Effect of a high-linolenic acid diet on lipogenic enzyme activities, fatty acid composition, and meat quality in the growing pig. Journal of Animal Science, 81(8):1967-79.

Kozlowska, J., Munoz, G. A. and Kolodziejczyk, P. P.(2008). Food and feed applications for flaxseed components. In: International conference on flax and other bast plants. Saskatoon, Canada, pp. 299307.

Kumar, A., Kumar, J., Singh, K. P.,Tomar and Sharma, P. K.(2018). Estimate heterosis for yield and yield components in Linseed (Linum usitatissimum L.) germplasm. International Journal of Current Microbiology and Applied Science,7(2): 2319-7706.
Kumar, A., Mishra, V. K., Vyas, R. P. and Singh, V. (2011). Heterosis and combining ability analysis in bread wheat (Triticum aestivum L.). Journal of Plant Breedingand Crop Science, 3(10): 209-217.

Kumari, K. and Rao, S.S. (2008). Genetic divergence in linseed (Linum usitatissimum L.). Mysore Journal of Agricultural Sciences, 42(1): 15-19.

Loria, R.M. (1993). Method of balancing oils and fats to enhance health. World Patent, WO9321774.

Mistry, C., Keshubhai, Kathiria, B., Sabolu, S. and Kumar, S.(2016). Heritability and gene effects for yield related quantitative traits in eggplant. Annals of Agricultural Science, 61(2): 237246.

Morris, D.H. (2007). Flax: A Health and Nutrition primer. Flax Council of India, pp. 1-112.

Nykter, M. and Kymalainen, H. R. (2006). Quality characteristics of edible linseed oil. Agriculture and Food Science, 15: 402-413.

Okorie, S.U and Nwachukwu, C.N. (2014). Comparative evaluation of quality characteristics of oils extracted from some selected legumes and a cereal. IOSR J. Environment Science Toxicology and Food Technology,8(7):2319-2402.

Patel, T. T. and Patidar, D.(2018). Study of high heterotic crosses in relation to combining ability and genetic divergent parents in Herbaceum Cotton. International Journal of Pure and Applied Bioscience, 6(1): 67-70.

Reddy, P. S. and Pati, D. (1995). Linseed package of practices for increasing production. Published by: The Project Director, Directorate of Oilseeds Research, Rajeiwiianagar, Hyderabad - 500030. 
Schaffer, P.S. and Holm, G.E. (1950). The Determination of Linoleic acid in milk fat. Journal of Dairy Science, 33(12): 865-869.

Science Daily. (2007). Flaxseed Stunts The Growth Of Prostate Tumors. Retrieved on 23 Nov. 2007.

Singh, K. K., Mridula, D., Rehal, J. and Barnwal, P. (2011). Flaxseed- a potential source of food, feed and fiber. Critical Review in Food Science and Nutrition., 51:210-222.

Singh, N., Chandrawati, Kumar, R., Kumar, S. and Yadav, H. K. (2016). Study on genetic combining ability estimates for yield and related traits in linseed (Linum usitatissiumum L.) Australian Journal of Crop Science, 10(11):15941600.

Talukder, M. Z. A., Karim, A. N. M. S., Ahmed, S. and Amiruzzaman, M. (2016). Combining ability and Heterosis on yield and its component traits in maize (Zea mays L.). Bangladesh Journal of Agricultural Reserach, 41(3): 565-577.

Thompson, L. U., Chen, J. M., Li, T., Strasser-Weippl, K. and Goss, P. E. (2005). Dietary flaxseed alters tumor biological markers in postmenopausal breast cancer. Clinical Cancer Research,11(10): 3828-35.

Warrand, J., Michaud, P., Picton, L., Muller, G., Courtois, B., Ralainirina, R. and Courtois, J. (2005). Flax (Linum usitatissimum) Seed cake: A potential source of high molecular weight arabinoxylans. Journal of Agricultural and Food Chemistry, 53: 1449-1452.

Yamanura, K., Madhusudan, K. and Nadaf, H. L. (2009). Combining ability and gene action for yield and yield components in sesame (Sesamum indicum L.). Karnataka Journal of Agricultural Science, 22(2): 255-260.

\section{How to cite this article:}

Anu Rastogi and Sudhir Shukla. 2019. Combining Ability Analysis for Oil and Seed Parameters in Linseed (Linum usitatissimum L.). Int.J.Curr.Microbiol.App.Sci. 8(01): 11181148. doi: https://doi.org/10.20546/ijcmas.2019.801.120 